\title{
An Experimental Investigation of Spall Fracture in Neat Epoxy and an Epoxy/Carbon Nanotube Composite
}

by

\section{Jonathan Pepper}

A thesis submitted to the Faculty of Graduate and Postdoctoral Affairs

in partial fulfillment of the requirements for the degree of

Master of Applied Science

in

Aerospace Engineering

Carleton University

Ottawa, Ontario

(C) 2017

Jonathan Pepper 


\section{Abstract}

The spall strength of a material can be used to parameterize its ability to resist tensile failure under high-strain-rate loading. Dynamic failure in an assortment of representative materials was studied. A focus was placed on characterizing the mechanical properties of a polymer-matrix composite. Load frame testing was carried out to measure mechanical properties at quasi-static strain rates, while plate impact experiments were conducted to examine material response under dynamic loading conditions. Successful identification of the spall strengths and fracture toughnesses of aluminum 6061-T6 and polymethylmethacrylate (PMMA) validated the methods used in this investigation. An effect of the curing agent on the spall strength of an epoxy was identified. Nanotubes were found to have a detrimental effect on the dynamic mechanical properties of the resulting nanocomposite. Fracture surfaces of recovered nanocomposite fragments were imaged using a scanning electron microscope to identify failure mechanisms and propose a failure process. 


\section{Acknowledgments}

I would like to begin by expressing my sincere gratitude for the guidance and support provided by my supervisor Professor Oren Petel. His passion for research and expert insight fueled my academic ambition. This work would not have been possible without his help, and for that, I thank him wholeheartedly.

Special thanks are extended to Meysam Rahmat and Behnam Ashrafi for generously sharing their results from the quasi-static load frame testing, for providing material specimens, and for offering their expertise on subjects including polymer science and composite materials. Financial support provided by the National Research Council Canada (NRC) through the Security Materials Technology (SMT) program is also greatly appreciated.

I am indebted to Andrew Higgins at McGill University for granting access to his research facility, and to Justin Huneault for his repeated assistance when conducting plate impact experiments.

Several individuals within the department of mechanical and aerospace engineering at Carleton University are deserving of my gratitude. First and foremost, I would like to thank Alex Proctor, Ian Lloy, and Kevin Sangster at the machine shop for providing their expert assistance when manufacturing critical experimental components. I would also like to thank Steve Truttmann, David Raude, and Stephan Biljan for their guidance relating to the design and installation of the gas gun.

And last but not least, I would like to thank my family and friends for their unwavering support throughout my studies. Their faith in my academic ability was critical to any success that I found as a student of engineering. I am indebted to them, forever and always. 


\section{Table of Contents}

$\begin{array}{ll}\text { Abstract } & \text { i }\end{array}$

$\begin{array}{ll}\text { Acknowledgments } & \text { ii }\end{array}$

Table of Contents iii

List of Tables

List of Illustrations vii

List of Symbols $\quad$ xii

1 Introduction $\quad 1$

1.1 The Relevance of Spall Testing . . . . . . . . . . . . . . . . . . 2

1.2 Armour Design . . . . . . . . . . . . . . . . . . . 3

1.3 Present Study . . . . . . . . . . . . . . . . . . 4

2 Previous Work $\quad 6$

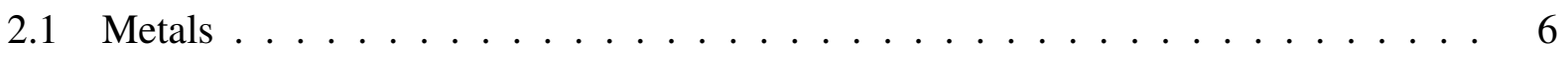

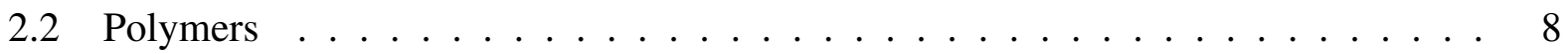

2.3 Polymer-Matrix Composites . . . . . . . . . . . . . . 11

3 Fundamentals of Shock Theory $\quad 13$

3.1 Shock Waves in Solids . . . . . . . . . . . . . . . . . . 13 
3.2 The Rankine-Hugoniot Jump Equations ～. . . . . . . . . . . . . . . . . 15

3.3 Slab Impact Mechanics . . . . . . . . . . . . . . . . . . . . . . . . . . 19

3.4 Spall Theory . . . . . . . . . . . . . . . . . . . . . . 24

4 Experimental Details $\quad 29$

4.1 Materials . . . . . . . . . . . . . . . . . . . . . . . . . . . 29

4.2 Load Frame Testing . . . . . . . . . . . . . . . . . . . . . . . . 32

4.3 Plate Impact Experiments . . . . . . . . . . . . . . . . . . . . 33

4.3 .1 Gas Gun . . . . . . . . . . . . . . . . . . . . 34

4.3.2 Target Fabrication . . . . . . . . . . . . . . . . 35

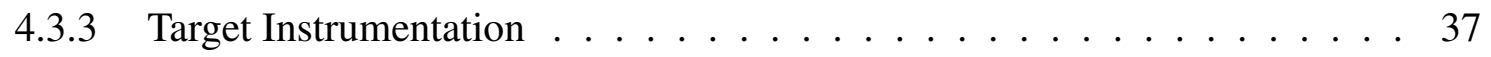

$4.3 .4 \quad$ Signal Processing . . . . . . . . . . . . . . . . . . . . . 40

5 Results and Discussion $\quad 43$

5.1 Aluminum $6061-\mathrm{T} 6 \ldots \ldots \ldots \ldots \ldots$. . . . . . . . . . . . . . . . . .

5.2 Polymethylmethacrylate $\ldots \ldots \ldots \ldots \ldots \ldots$

5.3 EPON 828 Epoxy . . . . . . . . . . . . . . . . . . . 51

$5.3 .1 \quad$ Strain Rate Effects . . . . . . . . . . . . . . . . . . . 57

5.3 .2 Effect of Curing Agent $\ldots \ldots \ldots \ldots \ldots$

5.4 Epoxy/Carbon Nanotube Composite $\ldots \ldots \ldots$. . . . . . . . . . 58

$5.4 .1 \quad$ Fracture Analysis . . . . . . . . . . . . . . . . . . . . 64

$\begin{array}{lr}\text { References } & \mathbf{7 0}\end{array}$

$\begin{array}{ll}\text { A Matlab Code } & \mathbf{7 6}\end{array}$

A.1 PDV Data Processing Script $\ldots \ldots \ldots \ldots \ldots$

A.2 Read LeCroy Binary Wave Waveform Script . . . . . . . . . . . . . . 78

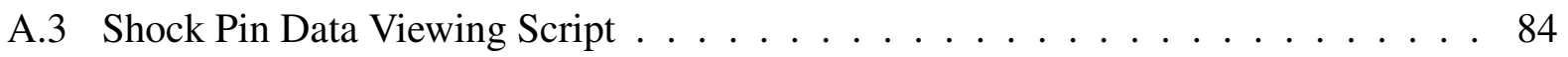

A.4 Coil Gauge Data Viewing Script $\ldots \ldots \ldots$ 
B Spectrogram Collection

C SEM Image Collection 


\section{List of Tables}

3.1 A summary of all the possible outcomes of the interactions between incident shock waves and rarefaction fans with a material interface. . . . . . . . . . 23

4.1 Acoustic properties of all relevant materials, where $\rho_{0}$ is bulk density, $C_{0}$ is bulk sound speed, $C_{L}$ is longitudinal sound speed, and $Z$ is shock impedance. . . . . . . 34

5.1 The results of the quasi-static uniaxial tensile experiments and the quasi-static fracture toughness experiments conducted by Meysam Rahmat and Behnam Ashrafi, respectively. STD refers to the standard deviation. . . . . . . . . . . . 43

5.2 The results of the plate impact experiments. . . . . . . . . . . . . 44 


\section{List of Illustrations}

1.1 Spall fracture in a slab of aluminum alloy caused by hypervelocity impact . . . . . 3

2.1 Brittle spall fracture in low-carbon steel . . . . . . . . . . . . . . . 8

2.2 Ductile spall fracture in pure Nickel . . . . . . . . . . . . . . . 8

2.3 Penny cracks resulting from spall fracture in polycarbonate . . . . . . . . . . . 9

3.1 A pressure wave traveling through an infinite solid . . . . . . . . . . . . . 14

3.2 Shocking-up of a pressure wave traveling through an infinite solid . . . . . . . . 14

3.3 The attenuation of a square shock wave . . . . . . . . . . . 15

3.4 A mass balance drawn across the shock front from the perspective of the shock coordinate system . . . . . . . . . . . . . . . . 16

3.5 The impact of two slabs . . . . . . . . . . . . . . . . . . . 19

3.6 The Eulerian position-time plot resulting from the impact of two slabs . . . . . . 20

3.7 Transmission and reflection resulting from a wave/interface interaction. . . . . . . 21

3.8 A Lagrangian position-time plot depicting the wave interactions that occur during a symmetrical plate impact $\left(Z_{\text {flyer }}=Z_{\text {target }}\right)$. Solid lines represent shock/compression waves, while dash lines represent rarefaction wavelets . . . . . . . . . 25

3.9 A schematic of the stress- $u_{p}$ plane that would result for the impact shown in Figure 3.8, where physical states (1)-(8) are shown in both plots to illustrate the nature of the stresses that develop in both bodies . . . . . . . . . . . . 25 
3.10 A Lagrangian position-time plot depicting the wave interactions that occur during an asymmetrical plate impact $\left(Z_{\text {flyer }}>Z_{\text {target }}\right)$. Solid lines represent shock/compression waves, while dash lines represent rarefaction wavelets . . . . . . . . 25

3.11 A schematic of the stress- $u_{p}$ plane that would result for the impact shown in Figure 3.10, where physical states (1)-(8) are shown in both plots to illustrate the nature of the stresses that develop in both bodies . . . . . . . . . . . . 25

3.12 A schematic plot of a free surface velocity profile from a spall experiment. . . . . . 26

4.1 The molecular structure of EPON 828 resin . . . . . . . . . . . . . . . 30

4.2 The load frame configured to compression for a fracture toughness test with a neat epoxy specimen. Photo courtesy of Behnam Ashrafi (NRC). . . . . . . . . . . . . 33

4.3 A schematic of the single-stage light gas gun. . . . . . . . . . . . . 35

4.4 The target mount with a fully-instrumented aluminum 6061-T6 target. . . . . . . 36

4.5 The sputter-coated side of a PMMA target showing a mirror finish. . . . . . . . 36

4.6 An EPON 828-A + P-CNT target for use in the plate impact experiments. . . . . . 37

4.7 The target mount with a fully-instrumented EPON 828-A + P-CNT target. . . . . . 37

4.8 A schematic of the target assembly, where $d=64 \mathrm{~mm}, r=25.4 \mathrm{~mm}, w_{f}=1.96$ or $1.55 \mathrm{~mm}$, and $w_{t}=3.02 \sim 6.60 \mathrm{~mm} \ldots \ldots \ldots$

4.9 A labeled photo of the target assembly. This particular setup did not include a

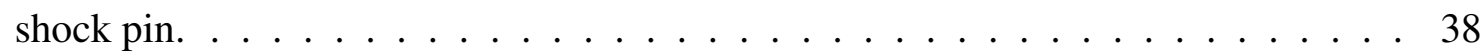

4.10 A schematic of a PDV system that depicts how the reference light (with frequency $f_{0}$ ) and the reflected light (with frequency $f_{d}$ ) are recombined at the detector . . . 39

4.11 An example of the voltage signals obtained from the electromagnetic coils during a shot with the gas gun. . . . . . . . . . . . . . . . 40

4.12 (a) The shock pin signal of an EPON 828 / EPIKURE 3223 epoxy +1 wt.\% P-CNT target impacted with an aluminum flyer plate at $617 \mathrm{~m} \cdot \mathrm{s}^{-1}$. (b) The waveform generated by the PDV system for the aforementioned impact, and (c) its associated

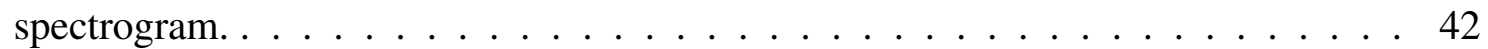


5.1 The spectrogram obtained from a plate impact experiment with an aluminum 6061T6 target impacted with an aluminum 6061-T6 flyer plate at a velocity of $633 \mathrm{~m} \cdot \mathrm{s}^{-1} .46$

5.2 Strength measurements for aluminum 6061-T6 plotted against Hugoniot state stress. 47

5.3 Strength measurements for aluminum 6061-T6 at low and high strain rates. . . . . 47

5.4 Fracture toughness measurements for aluminum 6061-T6 plotted against Hugoniot state stress. . . . . . . . . . . . . . . . . . . 48

5.5 Fracture toughness measurements for aluminum 6061-T6 at low and high strain rates. . . . . . . . . . . . . . . . . . . . 48

5.6 The spectrogram of a PMMA target impacted with an aluminum 6061-T6 flyer plate at a velocity of $502 \mathrm{~m} \cdot \mathrm{s}^{-1}$.

5.7 The spectrogram of a PMMA target impacted with a PMMA flyer plate at a velocity of $563 \mathrm{~m} \cdot \mathrm{s}^{-1}$

5.8 Strength measurements for PMMA plotted against Hugoniot state stress. . . . . . . 50

5.9 Strength measurements for PMMA at low and high strain rates. . . . . . . . . . . 50

5.10 A PMMA target impacted with an aluminum 6061-T6 flyer plate at a velocity of

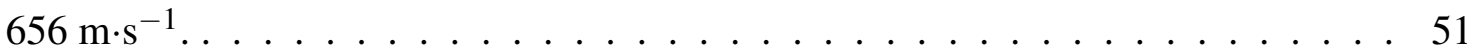

5.11 Fracture toughness measurements for PMMA plotted against Hugoniot state stress. 52

5.12 Fracture toughness measurements for PMMA at low and high strain rates. . . . . . 52

$5.13 U_{s}$ and $u_{p}$ measurements for both EPON 828 variants plotted with data by Carter and Marsh for comparison . . . . . . . . . . . . . . . . 53

5.14 An EPON 828-A target impacted with a PMMA flyer plate at a velocity of 136 $\mathrm{m} \cdot \mathrm{s}^{-1}$. A baseline velocity offset of $2984 \mathrm{~m} \cdot \mathrm{s}^{-1}$ was applied. . . . . . . . . . 54

5.15 An EPON 828-A target impacted with an aluminum 6061-T6 flyer plate at a velocity of $363 \mathrm{~m} \cdot \mathrm{s}^{-1}$.

5.16 An EPON 828-B target impacted with a PMMA flyer plate at a velocity of 344 $\mathrm{m} \cdot \mathrm{s}^{-1}$. 
5.17 An EPON 828-B target impacted with an aluminum 6061-T6 flyer plate at a velocity of $471 \mathrm{~m} \cdot \mathrm{s}^{-1} \ldots \ldots \ldots \ldots \ldots \ldots$

5.18 Strength measurements for EPON 828-A, EPON 828-B, and PMMA plotted against Hugoniot state stress. . . . . . . . . . . . . . . . . 55

5.19 Strength measurements for EPON 828-A and EPON 828-B at low and high strain

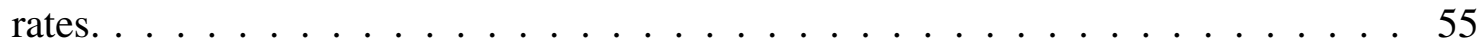

5.20 Fracture toughness measurements for EPON 828-A, EPON 828-B, and PMMA plotted against Hugoniot state stress. . . . . . . . . . . . . . . 56

5.21 Fracture toughness measurements for EPON 828-A and EPON 828-B at low and high strain rates. . . . . . . . . . . . . . . . . . 56

$5.22 U_{s}$ and $u_{p}$ measurements for EPON 828-A and the EPON 828-A + P-CNT composite plotted with data by Carter and Marsh for comparison . . . . . . . . . . . . 59

5.23 An EPON 828-A + P-CNT composite target impacted with an aluminum 6061-T6 flyer plate at a velocity of $617 \mathrm{~m} \cdot \mathrm{s}^{-1} \ldots \ldots \ldots$. . . . . . . . . . . 60

5.24 An EPON 828-A + P-CNT composite target impacted with a PMMA flyer plate at a velocity of $625 \mathrm{~m} \cdot \mathrm{s}^{-1} \ldots \ldots \ldots \ldots$. . . . . . . . . . . . . . . . . . .

5.25 An EPON 828 / EPIKURE 3223 epoxy + 1 wt.\% P-CNT composite target impacted with a PMMA flyer plate at a velocity of $346 \mathrm{~m} \cdot \mathrm{s}^{-1} \ldots \ldots$. . . . . . 61

5.26 An EPON 828 / EPIKURE 3223 epoxy + 1 wt.\% P-CNT composite target impacted with a PMMA flyer plate at a velocity of $483 \mathrm{~m} \cdot \mathrm{s}^{-1} \ldots \ldots \ldots 1$

5.27 Strength measurements for neat EPON 828-A and EPON 828-A + P-CNT composite plotted against Hugoniot state stress. . . . . . . . . . . . . . . . . 62

5.28 Strength measurements for neat EPON 828-A and EPON 828-A + P-CNT composite at low and high strain rates. . . . . . . . . . . . . . 62

5.29 Fracture toughness measurements for neat EPON 828-A and EPON 828-A + PCNT composite plotted against Hugoniot state stress. . . . . . . . . . . . . 63 
5.30 Fracture toughness measurements for neat EPON 828-A and EPON 828-A + PCNT composite at low and high strain rates. . . . . . . . . . . . . 63

5.31 SEM of a recovered nanocomposite target showing a top-down view of a fracture

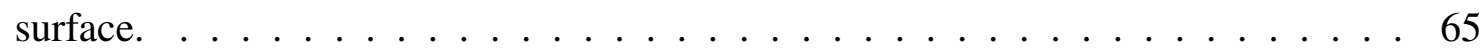

5.32 SEM of a recovered nanocomposite target showing a profile view of a fracture

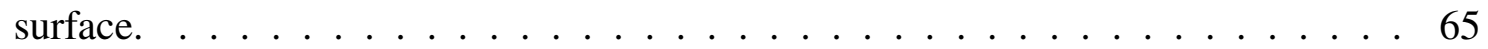

5.33 Fragments of a nanocomposite target recovered from a plate impact experiment. The free surface was spray painted with white enamel prior to impact. This made it easy to distinguish between internal fracture surfaces and the external free surface. 65

5.34 Geometry-induced shock strengthening in the focal area of a concave nanotube embedded in an epoxy matrix . . . . . . . . . . . . . . . 66 


\section{List of Symbols}

$\dot{\varepsilon} \quad$ Tensile strain rate

$\varepsilon_{H} \quad$ Hugoniot (shock) state strain

$\varepsilon_{\max } \quad$ Strain at maximum tensile stress

$\varepsilon_{y} \quad$ Strain at yield stress

$\rho \quad$ Density

$\rho_{0} \quad$ Bulk density

$\rho_{H} \quad$ Hugoniot (shock) state density

$\sigma_{H} \quad$ Hugoniot (shock) state stress

$\sigma_{\max }$ Maximum tensile strength

$\sigma_{s p} \quad$ Spall strength (Stepanov)

$\sigma_{s p}^{*} \quad$ Spall strength (Novikov)

$\sigma_{y} \quad$ Yield strength

C Sound (wave) speed

$C_{0} \quad$ Bulk sound speed

$C_{L} \quad$ Longitudinal sound speed 
d Gas gun bore diameter

E Elastic modulus

$e \quad$ Internal energy

$f_{0} \quad$ Reference laser frequency

$f_{d} \quad$ Disturbed (reflected) laser frequency

$K_{c} \quad$ Fracture toughness

$P \quad$ Pressure

$r \quad$ PDV probe spacing

$s \quad$ Non-dimensional parameter from the $U_{s}-u_{p}$ Hugoniot

$t \quad$ Time

$u_{p} \quad$ Particle velocity

$U_{s} \quad$ Shock velocity

$U_{t} \quad$ Strain energy density

$u_{f s} \quad$ Free surface velocity

$u_{\text {peak }}$ Peak velocity

$u_{\text {pull }} \quad$ Pullback velocity

$v \quad$ Volume

$v_{0} \quad$ Bulk volume

$v_{H} \quad$ Hugoniot (shock) state volume

$v_{i} \quad$ Impact velocity 
$w_{f} \quad$ Flyer plate thickness

$w_{t} \quad$ Target thickness

$x_{\text {coil }} \quad$ Coil gauge spacing

Z Shock impedance

HEL Hugoniot elastic limit

NRC National Research Council Canada

PDV Photonic Doppler velocimeter

SEM Scanning electron microscope

SMT Security Materials Technology

STD Standard deviation

TOI Time of impact 


\section{Chapter 1}

\section{Introduction}

Spall fracture in shocked materials is caused by the interaction of planar decompression waves (i.e., rarefaction fans) under the uniaxial strain condition, where inertial effects result in the formation of a region of tension inside a material body [1]. If the tensile stress achieved from such an interaction exceeds the local dynamic strength of the material, then fracture will occur. The minimum tensile stress that generates spall fracture in a material is defined as the spall strength of that material for that specific loading scenario. Planar decompression waves can be produced from the reflection of compressive waves at a stress-free surface, a scenario that can result from a high-velocity impact between two solids of finite geometry [1]. Tensile strain rates of $10^{4}$ to $10^{6} \mathrm{~s}^{-1}$ are typical for such loading conditions, while the dynamic tensile stresses achieved can be orders of magnitude greater that the quasi-static strengths of most materials [1].

The study of spall fracture in shocked materials is a field with significant relevance to the design of ballistic armour. While tensile strength can be measured with relative ease in the quasi-static and intermediate tensile strain rate domains, advanced techniques must be applied to measure strength at elevated tensile strain rates $\left(\dot{\varepsilon}>10^{4} \mathrm{~s}^{-1}\right)$. The classic spall test, which employs the plate impact experimental technique, can be used to measure the high-stress-state dynamic tensile strength (i.e., spall strength) of a material in a pure tension environment. The following work investigates the effects of various microstructural features on the spall strength of a selection of representative materials, with a focus on the effects of nanoparticle modification on the dynamic tensile strength and shock-response of thermosetting polymers. 


\subsection{The Relevance of Spall Testing}

The effects associated with high-velocity plate impact, close-proximity explosive detonation, and pulsed laser deposition of energy on a target can be methodically studied to provide insight on high-strain-rate deformation behaviour of materials [2]. While the primary goal of a spall test is to determine the spall strength of a material, a large assortment of experimental modifications and data analysis techniques can be used in parallel to extract additional information about other dynamic mechanical properties of the target. For example, spall testing can be used to study the strain-rate sensitivity of materials loaded in dynamic tension within the high-strain-rate regime (i.e., at strain rates exceeding $10^{4} \mathrm{~s}^{-1}$ ). It can also be used to identify shock-induced phase transitions and chemical reactions, as well as establish Hugoniot equations and map unloading equations of state (EOS). Furthermore, spall testing can be used to examine normal and shear wave propagation in shocked materials, as well as study wave/wave, wave/interface, and wave/microstructure interactions. Spall testing is also not limited to a single type of material. Dynamic tensile failure can be observed in metals (polycrystalline and single crystal), ceramics, polymers, composites, and bulk metallic glasses [2-6]. Spall even occurs in liquids, where failure is characterized by the formation of cavitation bubbles [7,8]. This diverse range of applications serves to fortify the status of spall testing within the shock physics community as a useful, reliable, and accurate method of studying deformation behaviour at high strain rates.

Spall strength (as a parameter) can be used to identify the onset of tensile failure in materials subjected high-strain-rate loading [8]. Knowledge of the spall strength of a material can be used in combination with other parameters such as dynamic shear strength, dynamic fracture toughness, hardness, Hugoniot elastic limit, stiffness, and ballistic limit to evaluate the capacity of a material to defeat a specific ballistic threat [9]. The application of spall theory to the design of ballisticallyrelevant materials and their use in modern armour systems is discussed in the following section. 


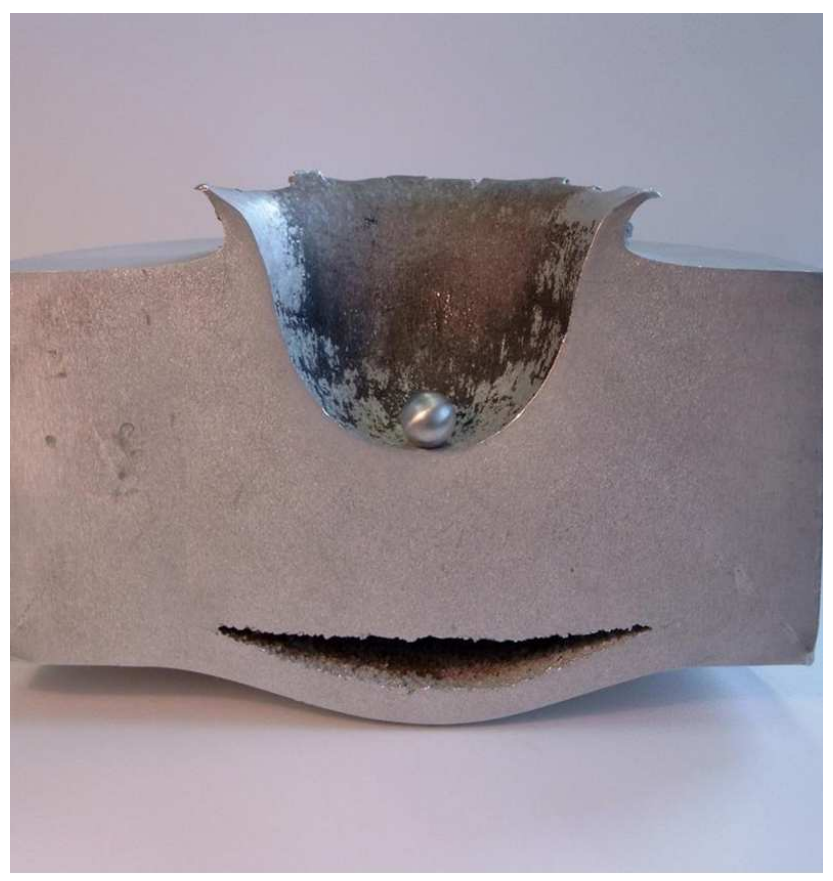

Figure 1.1: Spall fracture in a slab of aluminum alloy caused by hypervelocity impact [10].

\subsection{Armour Design}

The design of an armour system requires extensive knowledge of the response of the constituent materials under appropriate dynamic loading conditions. Comprehensive understanding of how a material will respond to both quasi-static and dynamic loading can be leveraged to devise methods of improving the ballistic performance of an armour system. The strain rates associated with ballistic impacts are often sufficient to produce spall fracture in an armour material. Therefore, the spall strength of the constituent materials provides a parameter that can be used to identify the onset of tensile failure, and hence, evaluate the ability of an armour system to resist ballistic impact. For example, Figure 1.1 depicts spall fracture in a slab of aluminum alloy resulting from the hypervelocity impact of a ball projectile [10].

The penetration of armour is the result of competing effects from several dynamic material properties at appropriate strain rates and stress states [9]. Knowing that microstructural features influence these critical mechanical properties to varying extents, it is therefore possible to modify a microstructure in a stand-in material to investigate how factors like processing techniques, particle 
inclusion, pressure-induced phase transitions, and deformation kinetics affect the aforementioned mechanical properties.

The strategies for selecting the appropriate materials for passive armour systems (as opposed to reactive or active protection systems) depend primarily on the nature of the ballistic threats that will likely be encountered. Modern passive armour systems generally consist of three functional layers: (1) a hard strike face to blunt, fragment, and/or erode the projectile, (2) an intermediate layer(s) with high energy-absorption and crack-arresting characteristics, and (3) a backing layer (ie., spall layer) designed to arrest fragments and prevent perforation [11].

Although significant research into the dynamic behaviour of metals and ceramics has been reported in the literature, historically, polymers and polymer-matrix composites have not received as much attention despite their widespread use in transparent, lightweight, and flexible armour. Only recently have developments in the production of high-performance matrix, particle, and fiber materials (of varying length scales) spurred an interest in research relating to the dynamic characterization of composite materials. Integrating foreign inclusions into a matrix without compromising structural integrity and promoting the formation of strong bonds between the matrix material and the inclusions is necessary to ensure sufficient transfer of dynamic stresses. Indeed, this presents a complex challenge. The goal of this study is therefore to explore the behaviour of these advanced composite materials under high-strain rate loading conditions to investigate the effects of microstructural features and particle inclusion on performance. Details of the scope and the goals of this study are described in the following section.

\subsection{Present Study}

The goal of this project was to investigate dynamic tensile failure in a nanocomposite material that has potential for use as a component in a modern armour system. This methodical investigation sought to examine dynamic deformation behaviour of a material fabricated via incorporation of an advanced nanoparticulate into a proven polymer matrix. The initial stages of the project involved 
establishing an experimental method that could be applied to generate reliable spall strength data for a variety of representative materials for validation purposes. Plate impact experiments were conducted to create the conditions necessary for spallation in aluminum 6061-T6, polymethylmethacrylate (PMMA), neat epoxy, and an epoxy/carbon nanotube composite. The performance of these materials was evaluated in terms of spall strength, dynamic fracture toughness, and shock response. A previous investigation [5] observed little variation between the spall strengths measured for a neat epoxy and an epoxy/carbon nanotube composite, however that study was limited to low shock stresses that are not relevant to most ballistic applications. Therefore, the objective of this investigation was to extend the current body of knowledge by measuring the spall strengths of neat epoxies and an epoxy/carbon nanotube composite at ballistically-relevant shock stresses.

The mechanical properties of these materials were also characterized under quasi-static loading conditions using a load frame. This work was conducted at the laboratory facilities of the National Research Council Canada (NRC) in Ottawa by Meysam Rahmat and Behnam Ashrafi. Funding for this work was provided, in part, through the Security Materials Technology (SMT) program. Mechanical properties of these materials obtained from this work were compared to results obtained from plate impact experiments to examine performance and failure behaviour across a range of strain rates.

The final portion of this project involved using a scanning electron microscope (SEM) to examine fracture surfaces of nanocomposite fragments recovered from the plate impact experiments. This work was also conducted at the laboratory facilities of the NRC. Features seen on these fracture surfaces were leveraged to propose a failure process that dominated fracture initiation under dynamic loading conditions. This failure process was used to explain the observed results. 


\section{Chapter 2}

\section{Previous Work}

Experimental investigations of spall fracture in dynamically-loaded materials began in the early $20^{\text {th }}$ century with the work of Hopkinson [12] who first correctly identified spall fracture when examining the failure behavior of mild steel impacted with wet gun-cotton [2]. Since then the collective global research effort has made significant progress towards understanding the mechanics of spall fracture. Similar efforts were committed to devising practical experimental methods for generating and studying dynamic tensile failure. The following sections describe previous work in the field of spall testing. A focus was placed on describing investigations that examined spall in metals, polymers, and polymer-matrix composites.

\subsection{Metals}

Following Hopkinson's early work, little additional research on spall fracture was performed until the 1950s when Rhinehart [13] used a modified split Hopkinson bar to identify the critical stress at which spall fracture occurrs in steel, brass, copper, and an aluminum alloy [2]. In the years leading up to the 1980 's, remarkable advances were made in understanding spalling of metals by a number of Western research groups, most notably: Sandia National Laboratories [14-22], the Los Alamos National Laboratory [23, 24], and the Stanford Research Institute [25-27].

Butcher et al. [14] were among the first to study the effects of stress history on spall in metals. They acknowledged that, in order to establish a comprehensive description of spall fracture, 
it was first necessary to consider the influence of the intensity and duration of the initial shockcompression that occurs prior to tensile loading. They employed direct explosive detonation and plate impact techniques to generate triangular and square tensile wave profiles, respectively, in aluminum 6061-T6. Previous work showed that the tensile stress required to generate spall in a variety of metals is lessened for square waves rather than triangular waves [14]. Their investigations using triangular waves showed that the spall strength was invariant to the duration of the tensile stress [14]. They also discussed evidence [28] that showed how not all metals exhibit dependence on the time endured at peak tension. Examination of recovered specimens revealed that spallation is a rate-dependent process, where different failure mechanisms can be activated depending on loading conditions, resulting in fracture surfaces with varying morphology. Plate impact experiments conducted by Stevens and Tuler [15] expanded upon these findings by studying the influence of shock-precompression on the dynamic tensile strength of aluminum 6061-T6. They used the onset of incipient spall as a criterion for examining damage, since incipient spall is defined as the lowest tensile stress (for given pulse duration) that produces tensile cracking [15]. Their work provided evidence that the tensile fracture stress (i.e., spall strength) of aluminum 6061-T6 is invariant to shock pre-compression stress [15], and is instead dependent on the tensile loading rate. Measurements of incipient spall strength in aluminum 6061-T6 obtained from gas gun experiments were on the order of $1.0 \pm 0.1 \mathrm{GPa}$, while measurements obtained from exploding foil experiments were on the order of $1.4 \pm 0.1 \mathrm{GPa}$.

A separate study by Stevens et al. [16] looked at spall fracture in aluminum single crystals. By applying dislocation theory to a kinematic model, they developed equations to predict the growth rate of voids caused by spallation [16]. Stevens and Pope [29] published a compendium of research relating to dynamic failure in metals. This documents included results from experimental work conducted by Shockey et al. at the Stanford Research Institute. Their work examined effects of microstructure on dynamic fracture in low-carbon steel and pure nickel, which exhibit brittle and ductile spall fracture, respectively. Images of the spall surfaces of recovered samples of these metals are shown in Figures 2.1 and 2.2. 


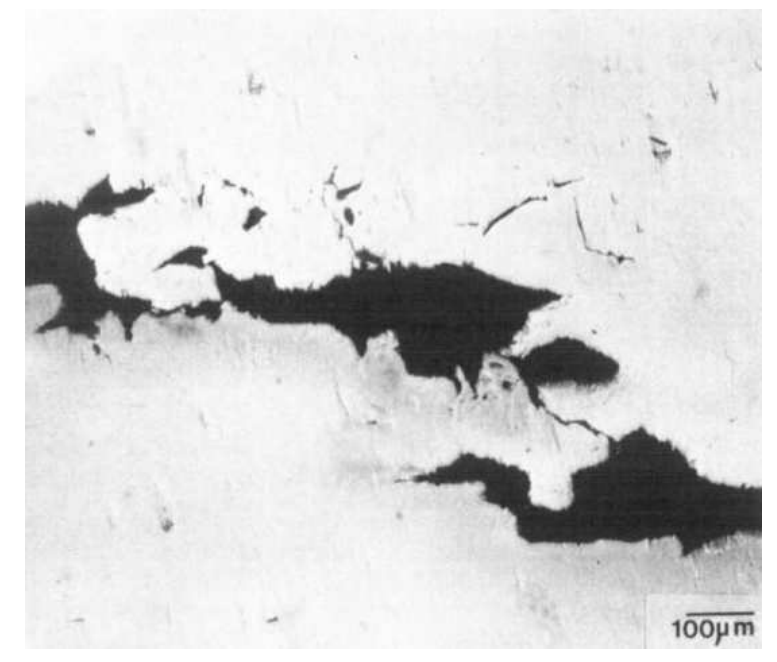

Figure 2.1: Brittle spall fracture in lowcarbon steel [29].

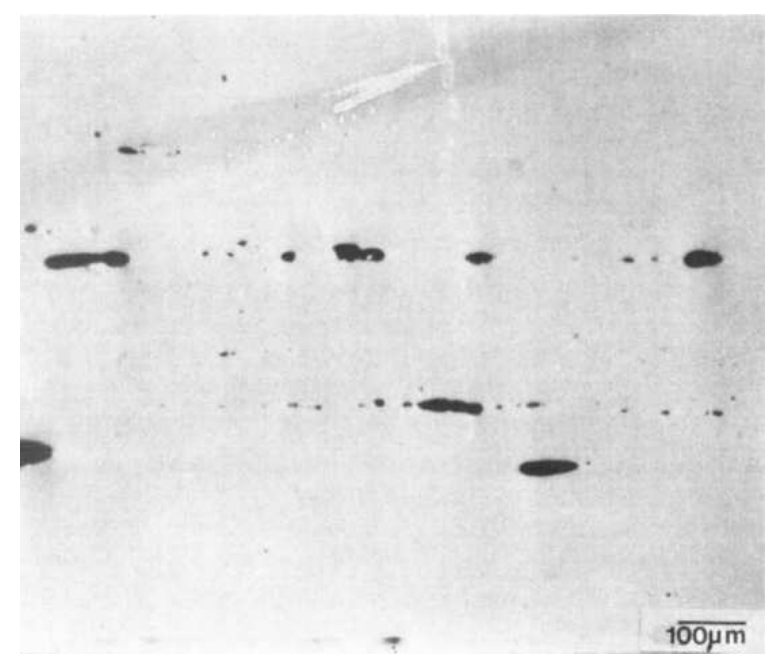

Figure 2.2: Ductile spall fracture in pure Nickel [29].

The majority of Western research on the dynamic fracture of metals conducted prior to 1980 has been elegantly summarized by Meyers and Aimone [2], and by Grady [30] in their respective review papers. Interested parties are directed to those documents for further reading. Dynamic tensile fracture has also been examined by Antoun et al. [8] in their (more recent) publication, which focuses on merging the results from research conducted in North American and the former Soviet Union to build a cohesive understanding of the mechanics of spall fracture.

\subsection{Polymers}

Early studies by Barker and Hollenbach [31] used plate impact experiments to study shock-wave propagation in polymethylmethacrylate (PMMA). They found that wave propagation was affected by nonlinearity in the material, strain-rate dependence, and elastic-plastic effects. More recent experimentation by Jordan et al. [32] confirmed and expanded upon their findings. Carter and Marsh [33] conducted explosive shock-loading experiments on a selection of representative polymers. In addition to establishing Hugoniot equations of state, they also observed a high-pressure transformation in all polymers characterized by a change in the slope of the $U_{s}-u_{p}$ Hugoniot, and occasionally a large decrease in volume. They attributed these observations to shock-induced re- 


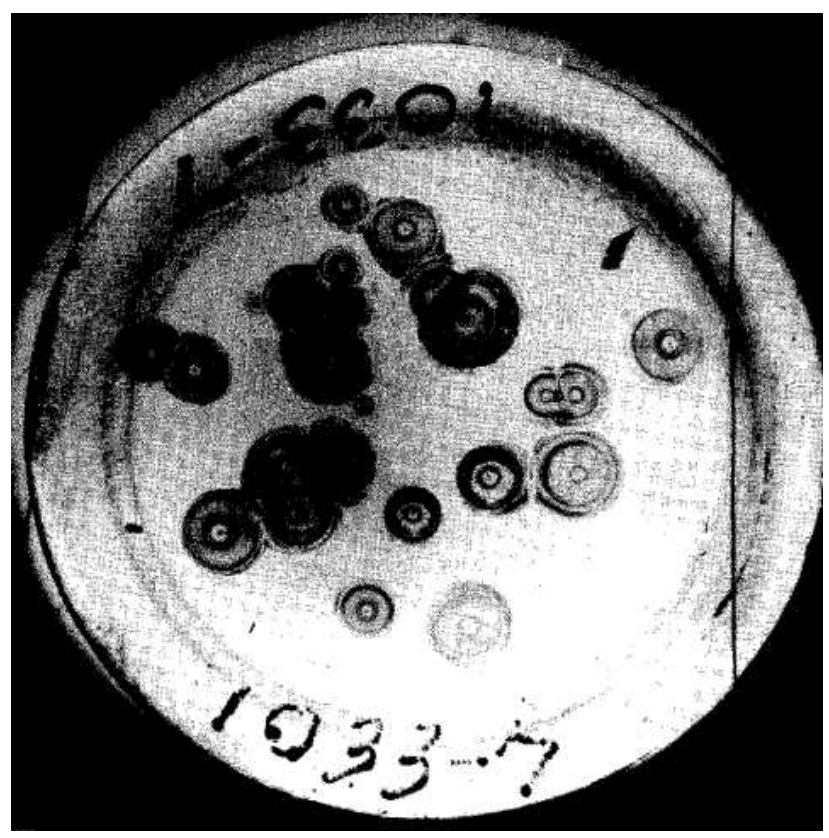

Figure 2.3: Penny cracks resulting from spall fracture in polycarbonate [25].

structuring of the molecular backbone, where the transformation parameters were shown to be insensitive to the degree of crystallinity of the original bulk polymer. They also ruled out the possibility of the transformation being caused by melting or vaporization, since the behaviour was observed in both thermosetting and thermoplastic polymers.

Chen et al. [34] used a modified split Hopkinson tension bar to study dynamic stress-strain responses and failure behaviour of PMMA and EPON 828/T-403 at strain rates on the order of $10^{3}$ $\mathrm{s}^{-1}$. They observed that the peak tensile strengths measured for EPON 828/T-403 under dynamic loading were achieved at smaller strains when compared to the quasi-static case. Furthermore, the specimens fractured in a brittle manner during dynamic tensile loading, while the quasi-static specimens failed in a ductile manner with a necking process, thereby providing qualitative evidence for a strain-rate effect. Plate impact experiments conducted by Curran et al. [25] were used to examine void nucleation and growth functions in polycarbonate by analyzing the incipient shock damage observed in recovered samples (see Figure 2.3), while Faye et al. [35] investigated the mechanics of dynamic fracture of polycarbonate relative to that of PMMA using combined numerical and experimental approaches. 
Millett et al. [36] conducted a thorough investigation into the effects of changing the chemistry of several polymers subjected to plate impact loading, where the electrostatic repulsion between adjacent polymer chains, as well as their molecular shape, were found to influence the shock response of the polymer. Millet et al. [37] also conducted plate impact experiments to study the behaviour of an epoxy resin under one-dimensional shock loading (impact velocities ranging from 201 to $\left.833 \mathrm{~m} \cdot \mathrm{s}^{-1}\right)$. By instrumenting the samples with longitudinal and lateral-oriented stress gauges, they were able to provide evidence for a shear strengthening effect similar to that observed in PMMA [38]. This result was attributed to the viscoelastic nature of epoxies.

Razack and Varghese [39] used thermogravimetric analysis (TGA), and differential scanning calorimetry (DSC) to investigate the effect of various hardeners (two aliphatic and two aromatic) on the properties of epoxy resin. Their results showed a clear divide in effectiveness between the thermal and mechanical performance of epoxy resin cured with aliphatic and aromatic hardeners, which is consistent with the work of Jain et al. [40] who studied the curing kinetics and thermal stability of epoxy.

Munson and May [41] studied the high-pressure compressibility of EPON 828 resin cured with three curing agents. The equilibrium compression curves for the three epoxy systems were essentially identical at pressures above $1 \mathrm{GPa}$, whereas the quasi-static results showed significant variation between systems. This effect suggests that variation in structure and the degree of crosslinking between polymer chains has a negligible effect on high-pressure compressibility. Differences in the quasi-static compression curves were attributed to variation in the equilibrium distances and binding forces between adjacent polymer chains, which are determined by the choice of curing agent and degree of cure.

An investigation by Golubev et al. [42] showed that the spall strengths of most amorphous polymers typically range between 100-300 MPa, where the observed spallation characteristics change with peak pressure. Samples recovered from plate impact experiments conducted at different temperatures showed zones of increasing plasticity near crack tips [42]. Despite observing a variety of fracture phenomena across a range of loading conditions, the spall strength of PMMA was shown 
to be invariant to increasing shock pressure [42] and only slightly sensitive to strain rate.

Spall fracture in epoxy has been studied by several researchers. Parhomenko and Utkin [43] published a spall strength of $300 \pm 50 \mathrm{MPa}$ for epoxy EDT-10 at an impact velocity of 850 $\mathrm{m} \cdot \mathrm{s}^{-1}$. Guess [44] reported a spall strength of $76 \mathrm{MPa}$ for EPON 828 epoxy (strain-rate not specified) in his unpublished work. While the dynamic tensile properties of these materials are well-documented, the effects of their microstructures on their performance under high-strain rate loading conditions is not well understood. Specifically, the effect of the curing agent on the spall strengths of thermosetting polymers presents a knowledge gap in the literature, where one could expect to see an influence of the microstructure on macroscopic mechanical performance.

\subsection{Polymer-Matrix Composites}

Several recent studies have focused on dynamic loading of thermosetting polymer-matrix composites [5, 45-47]. Research efforts include investigations on the influence of fiber orientation in carbon fiber-reinforced epoxy composites [47], the tensile strength of glass fiber composites under dynamic loading conditions [45], and the dynamic fracture of carbon nanotube epoxy composites under high strain rate loading [48]. The general consensus from this research is that the mechanical properties of such composites depend on several factors including the associated manufacturing process, the size, geometry, orientation, and distribution of the filler, the chemical composition of the materials, and the strength of the bond between the filler and the polymer matrix [5]. This array of factors contributes to the widespread variance in the experimentally-observed mechanical properties of these composites, which gives rise to the difficulty in predicting their properties under a range of loading conditions. Similar results have been observed for thermoplastic and elastomeric polymers [35, 49] and their composites [50]. From these investigations, it was found that the addition of fibers or nano-particulates into these polymers had either beneficial or detrimental effects on the observed mechanical properties of these systems, including quasi-static tensile strength, spall strength, and fracture toughness [5]. 
Fracture during high-strain rate loading of fiber-reinforced composites is often attributed to either partial or complete pullout with no fiber-breaking, whereas the observed dynamic tensile strength is proportional to the bonding strength between the fiber and the polymer matrix $[5,47,51]$. For nano-particulate polymer composites, fracture is attributed to the delamination of the particles from the matrix, which results in the formation of voids that can grow and coalesce into large cracks. For both nano-particulate and nano-fiber composites, spall strength and fracture toughness has been observed to increase at higher strain rates [5, 47]. It therefore becomes necessary to optimize the filler-matrix interface by controlling the volume fraction, orientation, and geometry of the filler material in order to improve the dynamic tensile properties of the composite. 


\section{Chapter 3}

\section{Fundamentals of Shock Theory}

In order understand the mechanics of spall fracture, one must first establish a working knowledge of shock theory. This begins by considering the shock-compression behaviour of solid materials. Once the fundamentals of shock theory are understood, only then is it practical to discuss the phenomena that result from the interactions of shock waves.

\subsection{Shock Waves in Solids}

Consider the longitudinal pressure wave propagating through a solid of infinite length represented in Figure 3.1. The undisturbed matter ahead of the wave exists at ambient conditions. Point A is a location in the solid that coincides with the front of the pressure wave and has a pressure $P_{A}$, a sound speed $C_{A}$, and particle velocity $u_{p, A}$. Looking now at point $\mathrm{B}$, we observe that the pressure wave has compressed the matter at location $\mathrm{B}$ to pressure $P_{B}$, where $P_{B}>P_{A}$. Let us assume that $P_{B}$ exceeds the pressure that defines the local elastic limit of the material, where the elastic limit is the pressure at which a material transitions from being purely elastic to being elastic-plastic. This implies that the pressure in this region is no longer linearly-related to wave speed [52]. The increase in pressure drives an increase in the local wave speed and particle velocity, where $C_{B}>C_{A}$ and $u_{p, B}>u_{p, A}$. Since the wave speed at B is faster than the wave speed at A, we know that the wave at B will eventually catch up to the wave at A, causing the wave front to straighten out to the point where it resembles a vertical line. This is the point where the pressure wave becomes a shock 


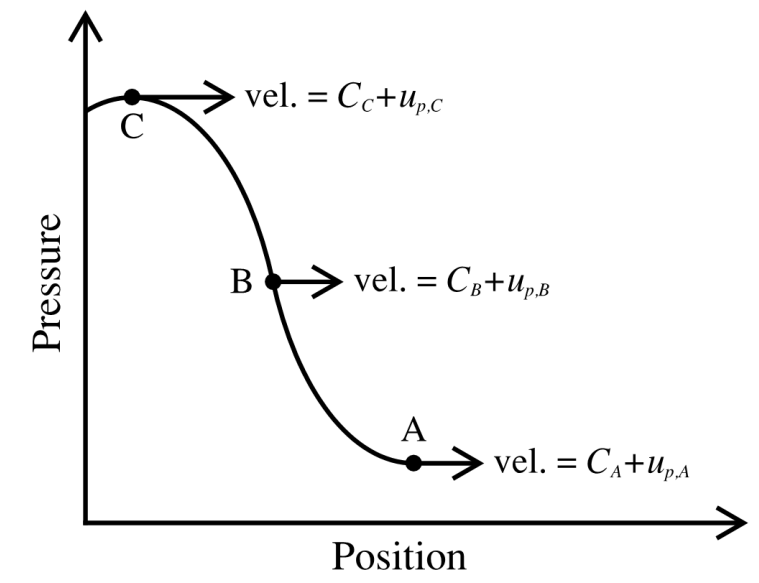

Figure 3.1: A pressure wave traveling through an infinite solid [52].

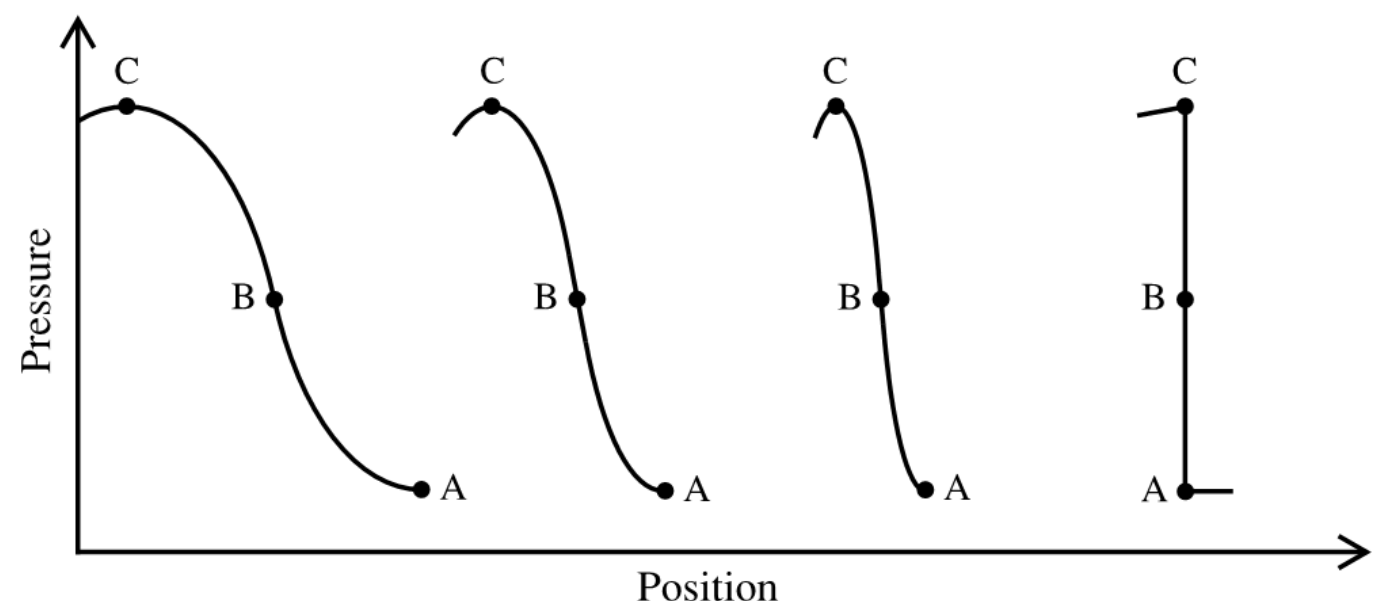

Figure 3.2: Shocking-up of a pressure wave traveling through an infinite solid [52].

wave. Here, the matter appears to instantaneously jump from ambient to shocked conditions as it crosses the wave front. The wave front now marks a discontinuity in the states of matter, where matter transitions non-isentropically from one equilibrim state to another as it crosses the wave front. The process of a pressure wave evolving into a shock wave is referred to as shocking-up [52]. This process is illustrated in Figure 3.2.

Let us now examine the fully-developed square shock wave propagating through a semi-infinite solid, represented as state (1) in Figure 3.3. The back of the wave (point C) is at ambient pressure and is moving into matter that has been shocked (i.e., matter that has non-isentropically jumped from a purely elastic state below the elastic limit to a state of elastic-platic or fully-plastic behaviour, generally associated with high pressure, density, and temperature). The wave speed in the 


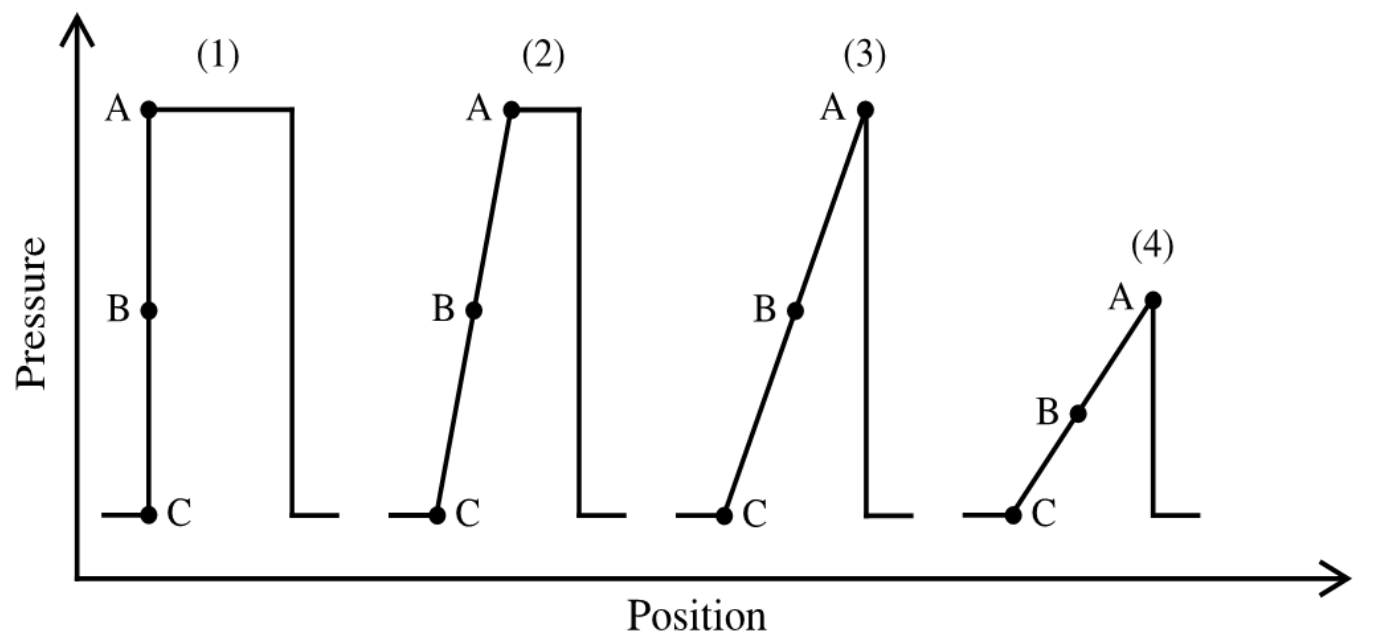

Figure 3.3: The attenuation of a square shock wave [52].

shocked state is proportional to density and non-linearly related to pressure. The shock front is traveling into matter at ambient conditions (i.e., lower density and slower wave speed). Therefore point $\mathrm{C}$ must be traveling slower than the wave front, while point $\mathrm{A}$ must be traveling faster than the shock front. This implies that point A will gradually catch up to the shock front, causing the pressure at the current position occupied by point $\mathrm{A}$ to diminish due to the influence of point $\mathrm{C}$. This is shown in state (2). As this process continues, the slope of the back of the wave will gradually taper off until point A catches up to the shock front, represented by state (3). Still traveling faster, the back of the wave will begin to attenuate the shock front, reducing the shock pressure and diminishing the intensity of the discontinuity, as seen in state (4). Attenuation of the shock front will proceed until the wave is eliminated entirely.

\subsection{The Rankine-Hugoniot Jump Equations}

Consider a reference frame that is fixed to a square shock front. From this perspective, the matter immediately ahead of the shock front would be at ambient conditions, while the matter immediately behind it would be in a shocked state. This reference frame is referred to as a shock coordinate system. A schematic depicting a mass balance drawn across a shock front from the perspective of the shock coordinate system is shown in Figure 3.4. From this prospective, there are five parame- 


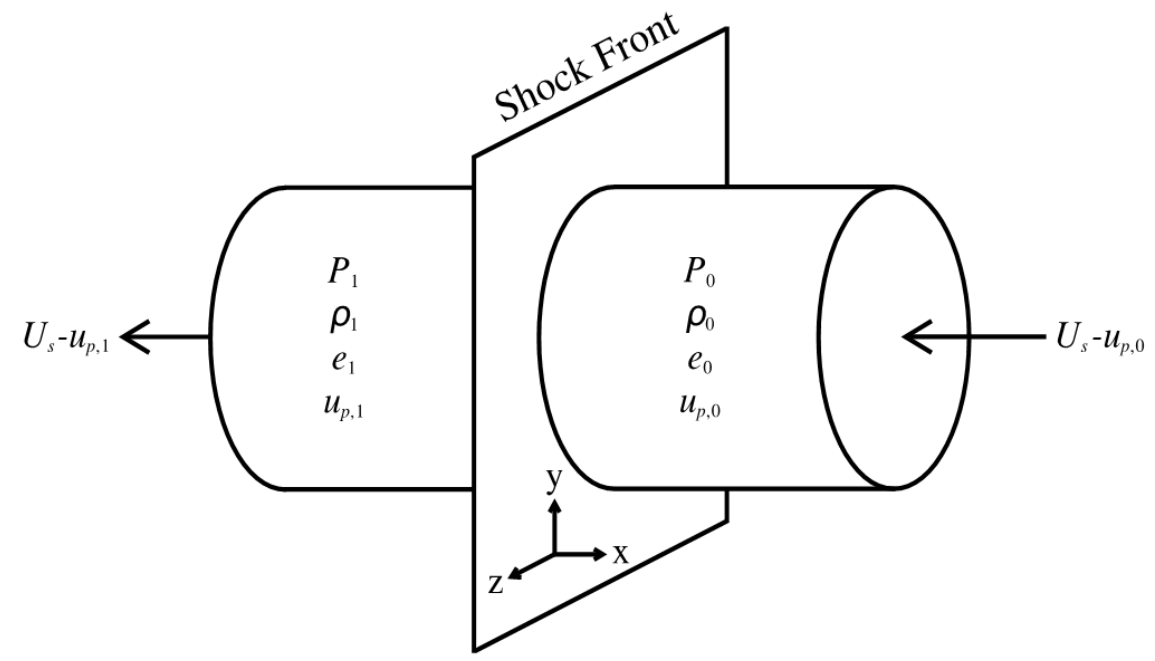

Figure 3.4: A mass balance drawn across the shock front from the perspective of the shock coordinate system [52].

ters that are needed to adequately define the conditions of the matter ahead and behind the shock front. These parameters include: shock velocity $\left(U_{s}\right)$, particle velocity $\left(u_{p}\right)$, density $(\rho)$, internal energy $(e)$, and pressure $(P)$. The relationships between these parameters ahead and behind the shock front, assuming one-dimensional loading, are described by the Rankine-Hugoniot jump equations. These relationships, which are derived from the conservation laws of mass, momentum, and energy across the shock front, are written as [52]

$$
\begin{gathered}
\frac{\rho_{1}}{\rho_{0}}=\frac{U_{s}-u_{p, 0}}{U_{s}-u_{p, 1}}=\frac{v_{0}}{v_{1}}, \\
P_{1}-P_{0}=\rho_{0}\left(u_{p, 1}-u_{p, 0}\right)\left(U_{s}-u_{p, 0}\right), \\
e_{1}-e_{0}=\frac{P_{1} u_{p, 1}-P_{0} u_{p, 0}}{\rho_{0}\left(U_{s}-u_{p, 0}\right)}-\frac{1}{2}\left(u_{p, 1}^{2}-u_{p, 0}^{2}\right) .
\end{gathered}
$$

Here states 1 and 0 represent the shocked and unshocked matter, respectively. In many cases the matter ahead of the shock front will be initially at rest, where $u_{p, 0}=0$. This knowledge can be applied to simplify Eqs. (3.1)-(3.3) to create a system of three equations and five variables. Two 
additional equations are therefore needed to close the system. Extensive experimentation [53] has shown that shock velocity is linearly-related to particle velocity in most materials, such that [52]

$$
U_{s}=C_{0}+s u_{p, 1}
$$

where $C_{0}$ is the bulk sound speed (i.e., a generalized sound speed for a material that accounts for the longitudinal and shear sound speed) and $s$ is a non-dimensional parameter. Empirical constitutive relationships in this form can be used in the place of equations of state, which are complex in solids, to close the Rankine-Hugoniot jump equations. Such expressions are known as Hugoniot equations of state [52], the most useful of which relate $U_{s}-u_{p}, P-v$, and $P-u_{p}$. Two Hugoniot equations can therefore be generated from experimental data and used in conjunction with Eqs. (3.1)-(3.2) to create a system of four equations and four variables, making it possible to solve for all the properties of the shocked matter.

The $U_{s}-u_{p}$ Hugoniot shown in Eq. (3.4) is especially practical because of its linearity. It is possible to devise a set of experiments that allows for direct measurement of the shock and particle velocities in a shocked solid. If the data obtained from these experiments is plotted and a linear trendline is fitted through the data series, then the y-axis intercept would equal the bulk sound speed $\left(C_{0}\right)$ and the slope of the trendline would equal the non-dimensional $s$ parameter. While this relationship applies to most elastic-plastic materials, it is not the case for many polymers, which often possess distinctly non-linear $U_{s}-u_{p}$ Hugoniots [31]. For these materials, analysis of shockcompression behaviour is typically achieved with the use of a quadratic or cubic trendline to fit the $U_{s}-u_{p}$ data series.

The $P-v$ Hugoniot equation is obtained by combining the $U_{s}-u_{p}$ Hugoniot with the mass and momentum jump equations, assuming $P_{0}=0$ and $u_{p, 0}=0$, such that [52]

$$
P_{1}=C_{0}^{2}\left(v_{0}-v_{1}\right)\left[v_{0}-s\left(v_{0}-v_{1}\right)\right]^{-2}
$$

It is necessary to emphasize that Hugoniot equations do not represent the path along which the ma- 
terial is stressed. Hugoniot equations approximate the isentropes that define a continuous loading path between separate states of matter, which in turn, describe a locus of the equilibrium states in which a shocked material can exist [52]. The line that connects the initial and final states on the $P-v$ Hugoniot therefore represents the jump condition. Such a line is called a Raleigh line [52]. If the particle velocity term is eliminated by manipulating the mass and momentum jump equations and letting $P_{0}=0$ and $u_{p, 0}=0$, then an equation of the linear Raleigh line connecting the initial and final states of matter can be written such that [52]

$$
P_{1}-P_{0}=\frac{U_{s}^{2}}{v_{0}}-\frac{U_{s}^{2}}{v_{0}^{2}} v_{1} .
$$

If the initial conditions are known, and the shock pressure $\left(P_{1}\right)$ and shock velocity $\left(U_{s}\right)$ are measured experimentally, then the final state volume $\left(v_{1}\right)$ can be determined. Conversely, if the initial and shocked states are known, then the shock velocity can be computed.

The last Hugoniot equation that will be discussed relates shock pressure $\left(P_{1}\right)$ and particle velocity $\left(u_{p, 1}\right)$. It is generated by manipulating the momentum jump equation and the $U_{s}-u_{p}$ Hugoniot to eliminate the $U_{s}$ term, once again assuming $P_{0}=0$ and $u_{p, 0}=0$, giving [52]

$$
P_{1}=\rho_{0} u_{p, 1}\left(C_{0}+s u_{p, 1}\right)
$$

Here it is crucial to recognize that, in this form, particle velocity is written in shock-fixed coordinates. In order to rewrite this expression in a laboratory (i.e., Eulerian) frame of reference, we must apply the Eulerian transform for particle velocity, where the $u_{p, 1}$ term is replaced with $\left(u_{p, 1}-u_{p, 0}\right)$. The slope of the Raleigh line connecting any of the states on the $P-u_{p}$ plane is a function of shock velocity, which is also written in Eulerian coordinates. Therefore when writing the $P$ - $u_{p}$ Hugoniot in its Eulerian form, the directionality of the shock velocity and the particle velocity must be considered relative to a laboratory frame of reference. For the case of a right-going shock, the Eulerian $P-u_{p}$ Hugoniot becomes [52] 


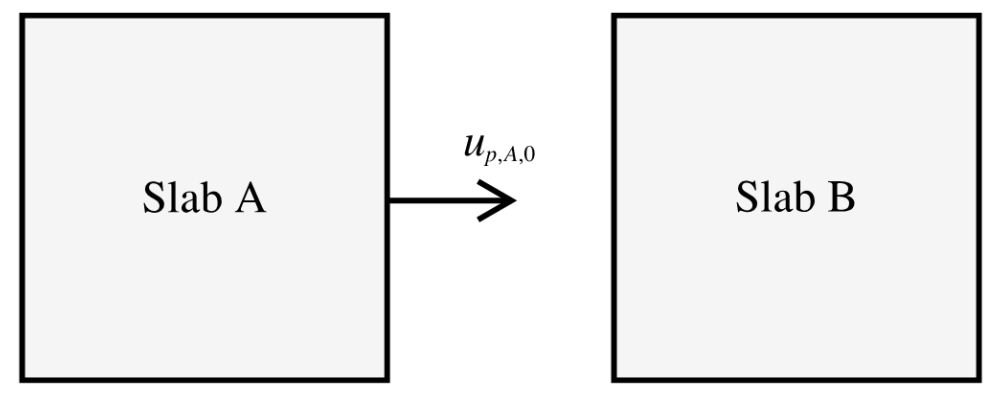

Figure 3.5: The impact of two slabs [52].

$$
P_{1}=\rho_{0} C_{0}\left(u_{1}-u_{0}\right)+\rho_{0} s\left(u_{1}-u_{0}\right)^{2} .
$$

For a left-going shock, the Eulerian $P$ - $u_{p}$ Hugoniot becomes [52]

$$
P_{1}=\rho_{0} C_{0}\left(u_{0}-u_{1}\right)+\rho_{0} s\left(u_{0}-u_{1}\right)^{2} .
$$

The Hugoniot equations of state can be used in conjunction with the Rankine-Hugoniot jump equations solve for the interaction of shock waves. This becomes especially important when trying to resolve the stress states in shocked materials with finite dimensions.

\subsection{Slab Impact Mechanics}

The mechanics of shock wave interactions will now be discussed, since ultimately spall fracture is a phenomenon that directly results from these interactions. Consider the impact of two solid slabs with finite size depicted in Figure 3.5, where slab A has an initial velocity of $u_{p, A, 0}$ and slab B is initially at rest. Prior to the impact, both slabs are at ambient conditions. At the instant of impact a shock pulse is generated at the interface between the slabs. The shock front propagates to the right in slab B and to the left in slab A. As long as the slabs maintain contact, the shocked material behind both wave fronts must be in equilibrium, meaning that the pressures and particle velocities behind the wave fronts in slab $\mathrm{A}$ and $\mathrm{B}$ are equal such that $P_{A, 1}=P_{B, 1}$ and $u_{p, A, 1}=u_{p, B, 1}$. This scenario is depicted in the position-time plot shown in Figure 3.6. From this result, the $P-u$ 


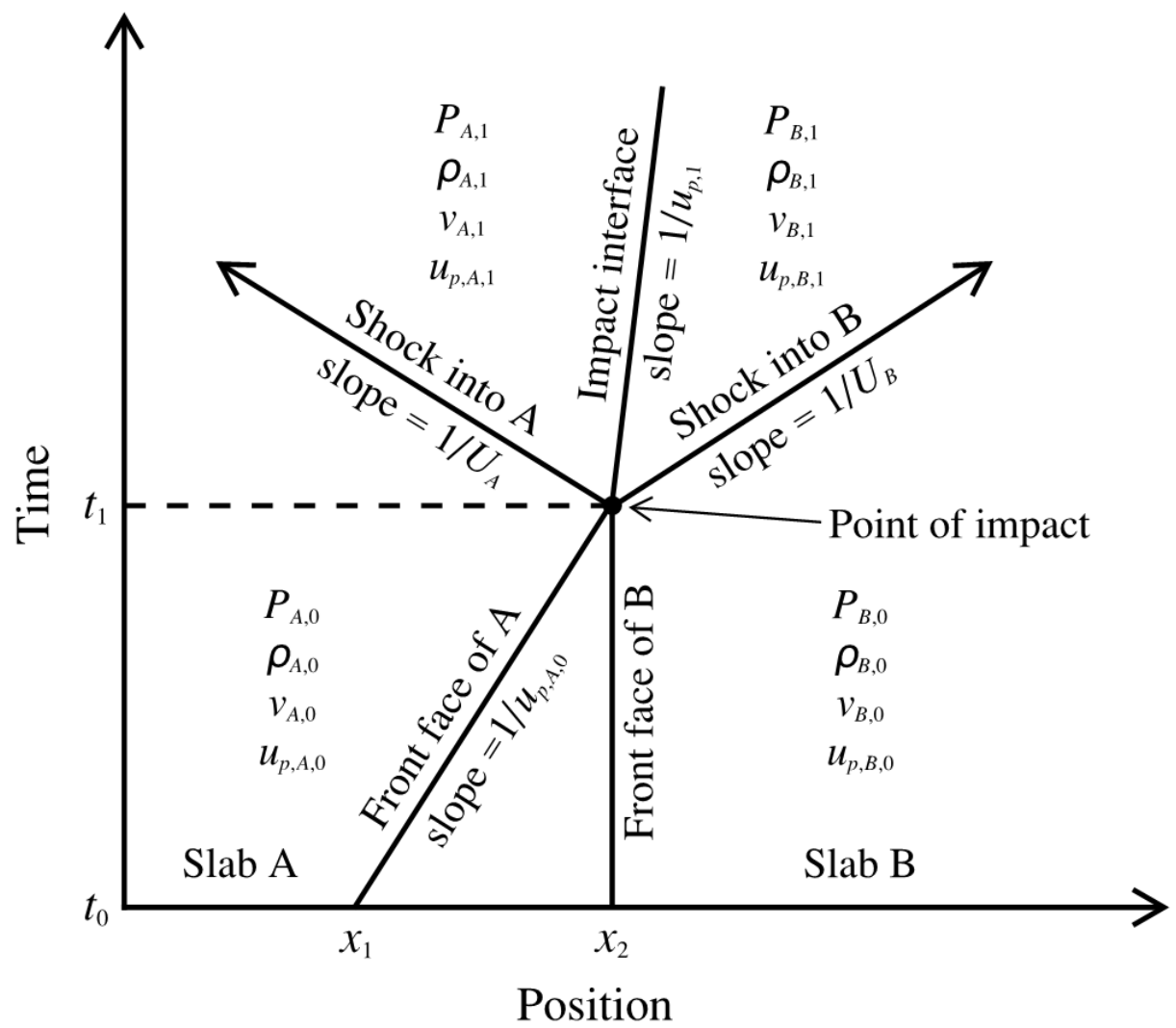

Figure 3.6: The Eulerian position-time plot resulting from the impact of two slabs [52].

Hugoniot for the right-going shock wave in slab B can be equated to the $P-u$ Hugoniot for the left-going shock wave in slab A. Since all the initial conditions of all materials are known, it is then possible to solve for the pressure and particle velocity of the shocked material. From there, the Eulerian shock velocity of slab A and B can be computed using [52]

$$
\begin{gathered}
U_{s, B}=\frac{P_{B, 1}}{\rho_{B, 0} u_{p, B, 1}}, \\
U_{s, A}=\frac{P_{A, 1}}{\rho_{A, 0}\left(u_{p, A, 1}-u_{p, A, 0}\right)}+u_{p, A, 0 .}
\end{gathered}
$$

The scenario in Figure 3.5 depicts the impact between two slabs of finite size. This means that the shock fronts propagating through slabs A and B will eventually encounter a surface that marks an interface between a solid and a gas. This interface is referred to as a free surface. At this point it is necessary to examine what happens when a shock wave meets an interface between two 


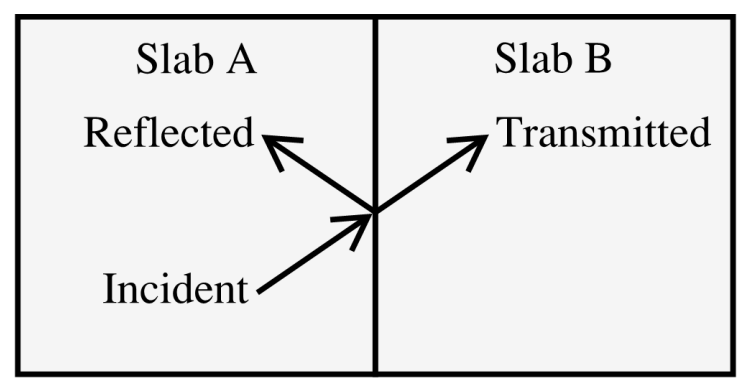

Figure 3.7: Transmission and reflection resulting from a wave/interface interaction.

different materials. Consider a shock wave passing through an interface between two conjoined slabs labeled A and B. This is depicted in Figure 3.7. When the shock wave meets the interface, it will be simultaneously transmitted and reflected. The properties of the transmitted and reflected wave depends on the relationships between the characteristic specific acoustic impedances (i.e., shock impedances) of slabs A and B. Shock impedance $(Z)$ is defined as [52]

$$
Z=\rho C
$$

where $\rho$ is density and $C$ is sound speed. Characteristic specific acoustic impedance is a measure of the opposition that a system presents to acoustic propagation resulting from the application of external pressure [54]. Conversely, shock impedance is a measure of the the opposition that a system presents to shock wave propagation. If $Z_{A}<Z_{B}$ then the incident wave will be reflected into slab A as a shock wave that further compresses the material from $P_{A, 1}$ to $P_{A, 2}$, where $P_{A, 2}>P_{A, 1}$ [52]. If, however, $Z_{A}>Z_{B}$ then a so-called rarefaction fan will be reflected into slab $\mathrm{A}$, where the rarefaction fan unloads the shocked material in slab A from $P_{A, 1}$ to $P_{A, 2}$, where $P_{A, 2}<P_{A, 1}$ [52]. A rarefaction fan is a continuous series of rarefaction waves (also known as unloading, decompression, or expansion waves) that unload a material from a state of high pressure and density to a state of low pressure and density. This unloading process is assumed to occur fast enough such that it can be considered adiabatic. Furthermore, since the process does not occur at absolute zero, thermodynamics dictates that unloading must also be isentropic [52]. Each unloading wave within the rarefaction fan travels at the local sound speed. Sound speed in solids is non-linearly 
proportional to pressure at pressures above the Hugoniot elastic limit (HEL) of the material [52]. Therefore, as the rarefaction fan continuously reduces the pressure of the material, the sound speed also decreases. Hence, the unloading waves at the front of the rarefaction fan travel faster than the unloading waves at the back, which causes the thickness of the rarefaction fan to increase over time. Rarefaction fans can be generated when a compressive shock wave meets a stress-free interface, commonly referred to as a free surface. Such a scenario can occur during an impact between two slabs of finite geometry. The arrival of the shock wave at a free surface accelerates the interface to a peak velocity, but has the corresponding effect of reflecting a rarefaction fan back into the material, which adiabatically and isentropically unloads the recently-shocked material back to ambient conditions.

Revisit, once again, Figure 3.7, but now consider what happens when the incident wave is instead a rarefaction fan. When a rarefaction fan meets an interface between two different materials, a transmission and reflection will result. Once again, the nature of the transmitted and reflected waves depend on the relationships between the shock impedances of the slabs $\mathrm{A}$ and $\mathrm{B}$. If $Z_{A}>Z_{B}$ then the incident rarefaction will be reflected back into slab A as a shock wave, re-compressing the recently-unloaded material from $P_{A, 1}$ to $P_{A, 2}$, where $P_{A, 2}>P_{A, 1}$. If, however, $Z_{A}<Z_{B}$, then the incident rarefaction fan will be reflected back into slab $\mathrm{A}$ as an intensified rarefaction fan. This will generate a tensile stress at the interface between slabs A and B. Since the slabs are merely pressed together, and the interface between them does not have a tensile strength, the slabs will separate. A summary of the interactions between shock waves and rarefaction fans with material interfaces is provided in Table 3.1 .

Now consider, yet again, the interaction of a shock wave with a free surface generated from the impact of two slabs of finite size. The shock impedance of any gas is significantly lower than that of any solid, so when the shock wave meets the free surface, a rarefaction fan will be reflected back into the slab that rapidly unloads the recently-shocked material to post-shock conditions. For the impact between slabs $\mathrm{A}$ and $\mathrm{B}$, the arrival of the left-going shock front at the free surface of A will reflect a right-going rarefaction fan back into the shocked slab. Similarly, a left-going 
Table 3.1: A summary of all the possible outcomes of the interactions between incident shock waves and rarefaction fans with a material interface.

\begin{tabular}{|l|c|c|c|}
\hline \multicolumn{4}{|c|}{ Incident Shock Wave } \\
\hline & $Z_{A}<Z_{B}$ & $Z_{A}=Z_{B}$ & $Z_{A}>Z_{B}$ \\
\hline Transmitted & Shock wave & Shock wave & Shock wave \\
\hline Reflected & Shock wave & - & Rarefaction fan \\
\hline \multicolumn{4}{|c|}{ Incident Rarefaction Fan } \\
\hline & $Z_{A}<Z_{B}$ & $Z_{A}=Z_{B}$ & $Z_{A}>Z_{B}$ \\
\hline Transmitted & Rarefaction fan & Rarefaction fan & Rarefaction fan \\
\hline Reflected & Rarefaction fan & - & Shock wave \\
\hline
\end{tabular}

rarefaction fan will be reflected from the free surface of slab B, created by the arrival of the initial right-going shock wave. We therefore know that the right-going rarefaction fan in $\mathrm{A}$ and the left-going rarefaction fan in B will eventually collide. If, for some reason, Slab B was thicker than slab A, then the collision between these two rarefaction fans would occur at a plane located somewhere within slab B. The interaction between these two planr rarefaction fans would generate a tensile stress state [8]. If this tensile stress exceeded the local dynamic tensile strength of the material, then partial or complete fracture will occur. Fracture generated in this manner is called spall fracture $[2,3,8,55,56]$. The degree of damage observed at the so-called spall plane can be used to characterize the failure as either incipient spall, partial spall, or total spall (listed in order of increasing severity) [2]. The magnitude of the minimum tensile stress that produces spall fracture (for a given loading condition) is designated as the spall strength of the material $[3,8]$. The strain rates associated with shock wave interactions, and the subsequent interactions of rarefaction fans, are quite high. Typical tensile strain rates associated with high velocity impacts between slabs range between $10^{4}$ to $10^{6} \mathrm{~s}^{-1}$ [1]. Therefore, spall fracture can be defined as dynamic uniaxial tensile failure caused by the interaction of planar rarefaction waves within a material [2].

The location of the spall plane is determined by several factors including the relationship between the shock impedances of slabs $\mathrm{A}$ and $\mathrm{B}$, the dimensions of the slabs, and the local wave speeds. Spall strength is often used in combination with parameters such as fracture toughness, ballistic limit, energy absorption, stiffness, and hardness to quantify resistance to dynamic failure caused by ballistic impact [9]. The following section describes how the analysis of the wave in- 
teractions generated from the impact of a solid flyer plate and a stationary target can be used to determine the spall strength of the target material.

\subsection{Spall Theory}

Consider a high-velocity impact between a flyer plate and a stationary target, where $Z_{\text {flyer }}=Z_{\text {target }}$. Such an impact can be described as a symmetrical impact, since it involves a collision between two materials with equal shock impedances $(Z)$. Conversely, an asymmetrical impact involves a collision between two materials with different shock impedance, where $Z_{\text {flyer }} \neq Z_{\text {target }}$. The wave interactions that result within both bodies during symmetrical and asymmetrical impacts are illustrated in Figure 3.8 and Figure 3.10, respectively. Note that material interface motion is neglected in these schematics. As discussed previously, the interactions of the right and left-going rarefaction fans will result in the generation of a large tensile stress within the target. If this tensile stress exceeds the local strength of the material, spall fracture will occur.

The location within the target where the two rarefaction fans will interact is determined by two factors: (1) the thicknesses and (2) the shock impedances of the flyer plate and the target. These factors also influence the shape of the wave fronts, and therefore effect material motion. Given that spall fracture is the result of wave interactions involving significant material motion, the spall strength of a material is determined from the analysis of the velocity history of the back (free) surface of the target. The evolution of the velocity history of the free surface are directly representative of the interactions between the shock waves, rarefaction fans, and material interfaces that occur during impact $[2,8]$. The effect of the flyer plate material on the wave interactions can be seen by comparing the features of the position-time diagrams in Figures 3.8 and 3.10, which depict a symmetrical and an asymmetrical impact, respectively. Here we see differences between the timing of the rarefaction interaction events, as well as a shift in the position of the spall plane.

The stress- $u_{p}$ Hugoniot planes associated with the impacts illustrated in Figures 3.8 and 3.10 are provided in Figures 3.9 and 3.11. States (1) and (2) represent the initial conditions of the flyer 


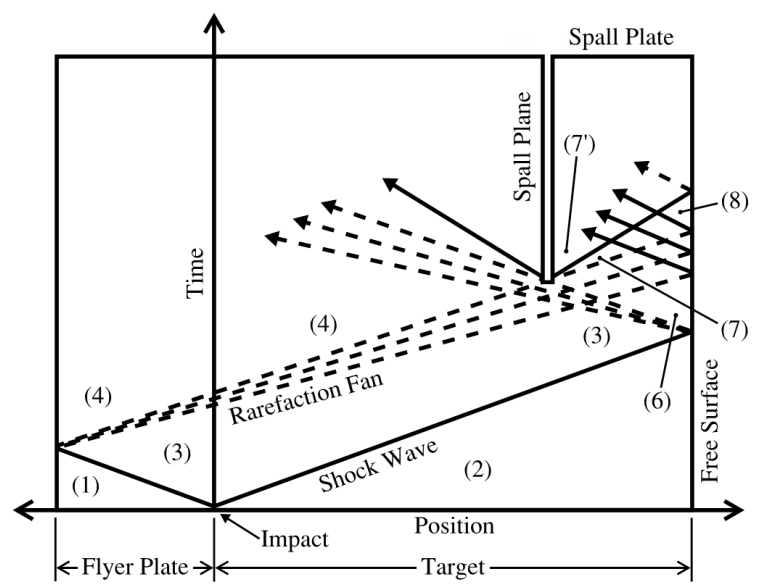

Figure 3.8: A Lagrangian position-time plot depicting the wave interactions that occur during a symmetrical plate impact $\left(Z_{\text {flyer }}=\right.$ $\left.Z_{\text {target }}\right)$. Solid lines represent shock/compression waves, while dash lines represent rarefaction wavelets [6].

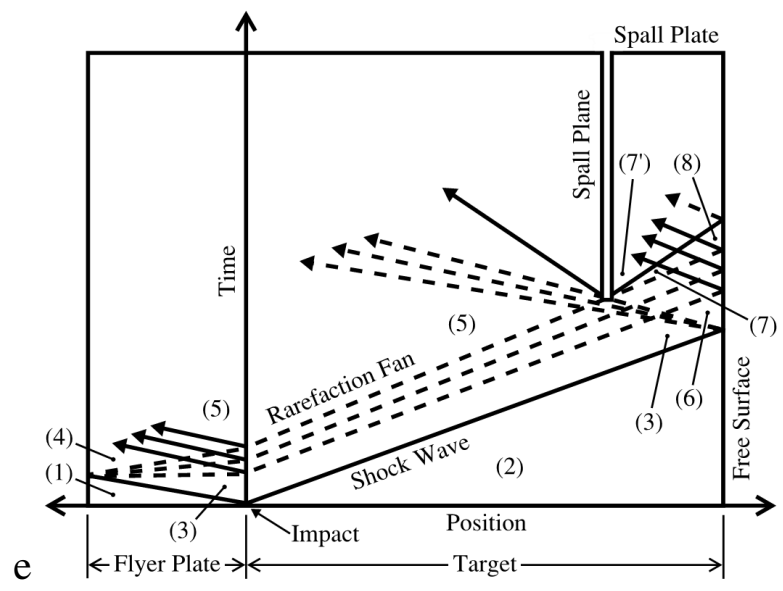

Figure 3.10: A Lagrangian position-time plot depicting the wave interactions that occur during an asymmetrical plate impact $\left(Z_{\text {flyer }}>Z_{\text {target }}\right)$. Solid lines represent shock/compression waves, while dash lines represent rarefaction wavelets [6].

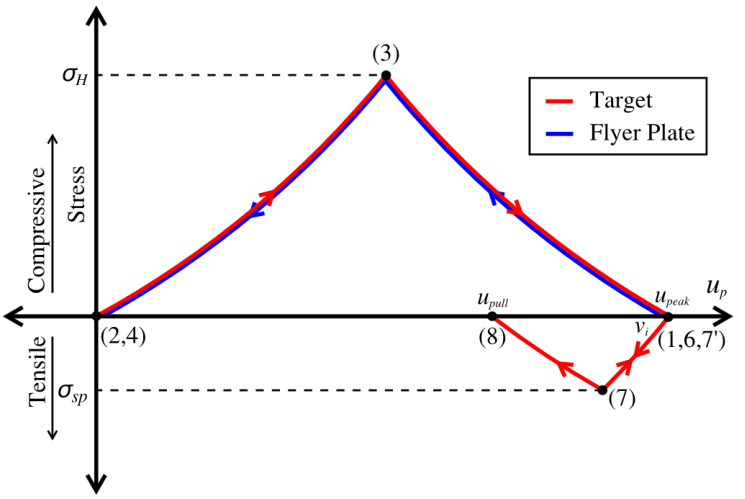

Figure 3.9: A schematic of the stress- $u_{p}$ plane that would result for the impact shown in Figure 3.8, where physical states (1)-(8) are shown in both plots to illustrate the nature of the stresses that develop in both bodies [6].

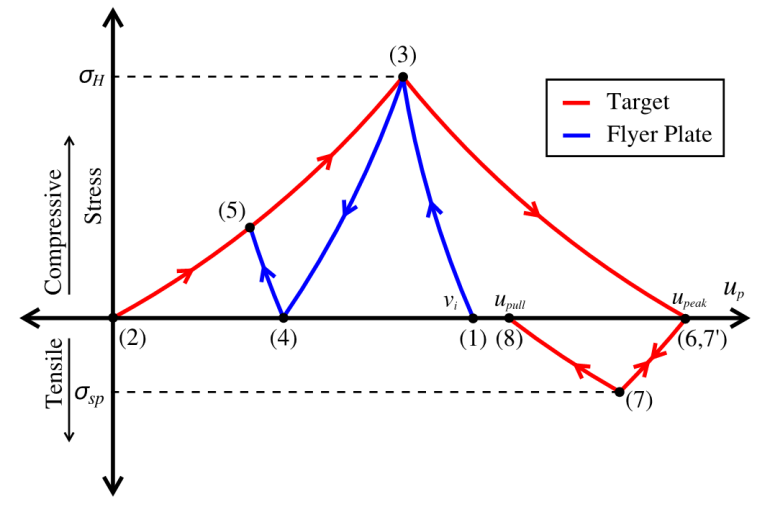

Figure 3.11: A schematic of the stress- $u_{p}$ plane that would result for the impact shown in Figure 3.10, where physical states (1)-(8) are shown in both plots to illustrate the nature of the stresses that develop in both bodies [6].

plate and target, respectively. State (3) represents the material state resulting from shock compression, where the Hugoniot stress (i.e., shock stress) is achieved. States (4) and (5) represent intermediary states of the flyer plate resulting from various wave/interface interactions. The particle velocity associated with state (6) is the peak velocity of the free surface $\left(u_{\text {peak }}\right)$. The stress 


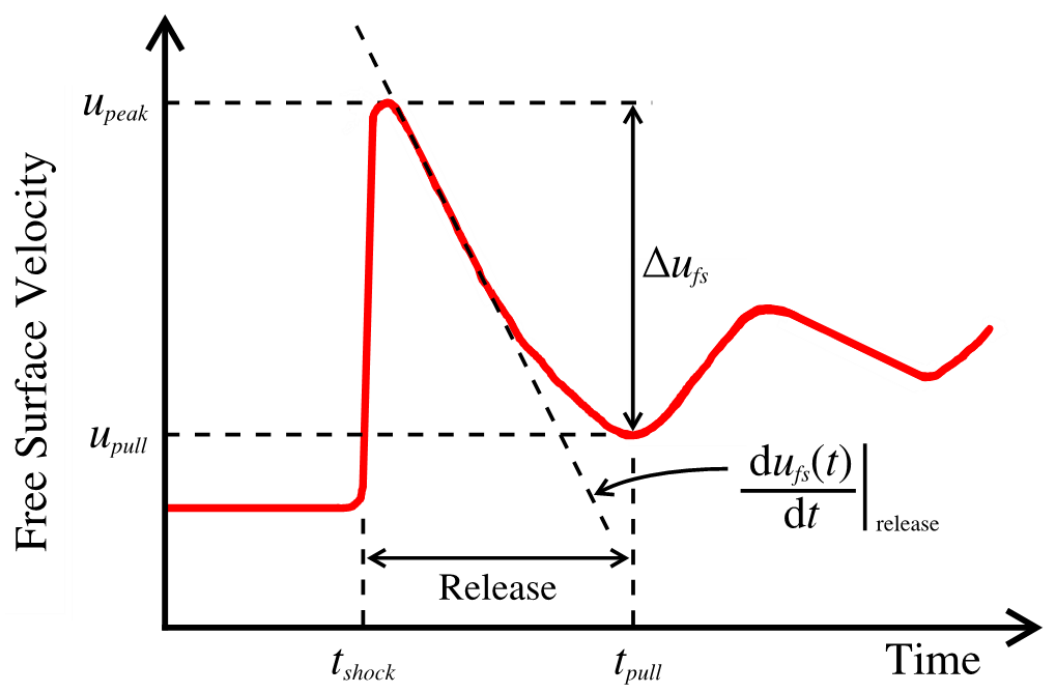

Figure 3.12: A schematic plot of a free surface velocity profile from a spall experiment.

achieved in state (7) is the tensile stress that produces incipient spall fracture of the target material. The magnitude of that stress therefore represents the spall strength of the target for that specific loading scenario. Finally, the particle velocity associated with state (8) is the pullback velocity of the free surface $\left(u_{\text {pull }}\right)$.

A generic example of a free surface velocity profile is shown in Figure 3.12. The post-shock particle velocity $\left(u_{p}\right)$ of the target (i.e., the particle velocity associated with state (3) in Figures 3.9 and 3.11), as observed from the free surface, is given by [52]

$$
u_{p} \approx \frac{1}{2} u_{\text {peak }} .
$$

Based on the wave interactions at the free surface, the velocity of the initial shock wave $\left(U_{s}\right)$ that is transmitted through the target can be determined from the transit time of the wave through the sample. In the case of a triangular shock front, $U_{s}$ more accurately describes the average velocity of the shock wave [52]. The experimentally-determined values of $U_{s}$ and $u_{p}$ can be used to generate the $U_{s}-u_{p}$ Hugoniot equation for the target material [52]. The Hugoniot state stress $\left(\sigma_{H}\right)$ and corresponding strain $\left(\varepsilon_{H}\right)$ achieved in the shocked target can be determined from the uniaxial strain condition, such that $[52,53,57]$ 


$$
\begin{gathered}
\sigma_{H}=\rho_{0} U_{s} u_{p}, \\
\varepsilon_{H}=1-\frac{\rho_{0}}{\rho_{H}}=\frac{v_{H}-v_{0}}{v_{0}}=\frac{u_{p}}{U_{s}},
\end{gathered}
$$

where $\rho_{0}$ and $v_{0}$ are bulk density and volume, respectively, and $\rho_{H}$ and $v_{H}$ are the density and volume of the shocked state, respectively. Note that it is necessary to assume steady wave propagation (i.e., where all parts of the wave front travel at identical velocities) when using Eq. (3.14) and (3.15) to analyze viscoelastic materials [41]. The spall strength $\left(\sigma_{s p}^{*}\right)$ of the target material can be predicted from the free surface velocity profile using an acoustics approach that gives the following linear approximation provided by Novikov $[8,56,58]$

$$
\sigma_{s p}^{*}=\frac{1}{2} \rho_{0} C_{L} \Delta u_{f s}
$$

where the characteristic change in the free surface velocity $\left(\Delta u_{f s}\right)$ is given by the difference between the observed peak velocity $\left(u_{\text {peak }}\right)$ and pullback velocity $\left(u_{\text {pull }}\right)$

$$
\Delta u_{f s}=u_{p e a k}-u_{p u l l}
$$

An alternative relationship for spall strength was proposed by Stepanov [59], where

$$
\sigma_{s p}=\rho_{0} C_{L} \Delta u_{f s} \frac{1}{1+\frac{C_{L}}{C_{0}}} .
$$

This relationship, rather than Eq. 3.16, is used in the present study to calculate spall strength since it better accounts for effects of elastic-plastic behaviour in the sample [8]. Therefore any value for spall strength provided henceforth in this report will have been computed using Eq. 3.18. The tensile strain rate $(\dot{\varepsilon})$ achieved during the release stage is approximated by the expression [60]

$$
\dot{\varepsilon} \approx-\left.\frac{1}{2 C_{0}} \frac{\mathrm{d} u_{f s}(t)}{\mathrm{d} t}\right|_{\text {release }}
$$


where the $\frac{\mathrm{d} u_{f s}(t)}{\mathrm{d} t}$ term is defined in Figure 3.12. Using the magnitudes of $\sigma_{s p}$ and $\dot{\varepsilon}$, it is possible to calculate the fracture toughness $\left(K_{c}\right)$ of the target using an algebraic manipulation of the model proposed by Grady for brittle spall fracture [30] such that

$$
K_{c}=\sqrt{\frac{\sigma_{s p}^{3}}{3 \rho_{0} C_{0} \dot{\varepsilon}}} .
$$

This model assumes that spall fracture in the target occurs in a brittle manner (i.e., through the lineup of a network of cracks to create an internal free surface) [30].

Significant qualitative information about the spall fracture process can also be determined from the analysis of a velocity profile obtained from a plate impact experiment. The frequency and rate of decay of the free-surface velocity oscillations, as well as features of the spall signal convey information regarding the spalling parameters. For example, the rise time of the spall signal (observed between the time of the initial velocity pullback and the secondary peak velocity) is directly coupled with the rate of fracture development [8]. Additionally, decay of the free-surface velocity oscillations within the spall plate are related to the damping characteristics of the interior fracture surface [8]. Such observations can be quantified using advanced analytical techniques that facilitate the interpretation of free surface velocity histories and spall signals [8]. 


\section{Chapter 4}

\section{Experimental Details}

\subsection{Materials}

A variety of representative materials were selected for experimentation. These include: aluminum alloy 6061-T6, polymethylmethacrylate (PMMA), neat epoxy, and an epoxy-matrix nanocomposite. The purpose of this section is to provide background information that is relevant to the study of the failure behaviour of these materials under quasi-static and dynamic loading conditions. Details of these materials are provided below.

Aluminum 6061-T6 was the first material to be tested. It was chosen because of its widespread availability and because measurements of its spall strength $\left(\sigma_{s p}\right)$ and fracture toughness $\left(K_{c}\right)$ are well-documented, which meant that the results of the impact experiments could be compared to values previously reported in the literature. Successful identification of these parameters would validate the experimental method used to generate the data and the analytical method used to process it. Load frame testing was not performed for aluminum 6061-T6 under quasi-static loading conditions, since its mechanical properties are well-documented.

The next material to be tested was PMMA, which is a transparent, amorphous thermoplastic more commonly referred to as acrylic or Plexiglas ${ }^{\circledR}$. It was tested as a means of validating our experimental techniques for use on polymers by comparing our results with data reported previously in the literature. Load frame testing was not performed for PMMA under quasi-static loading conditions, since values of its $\sigma_{\max }$ and $K_{c}$ are well-documented [61, 62]. 


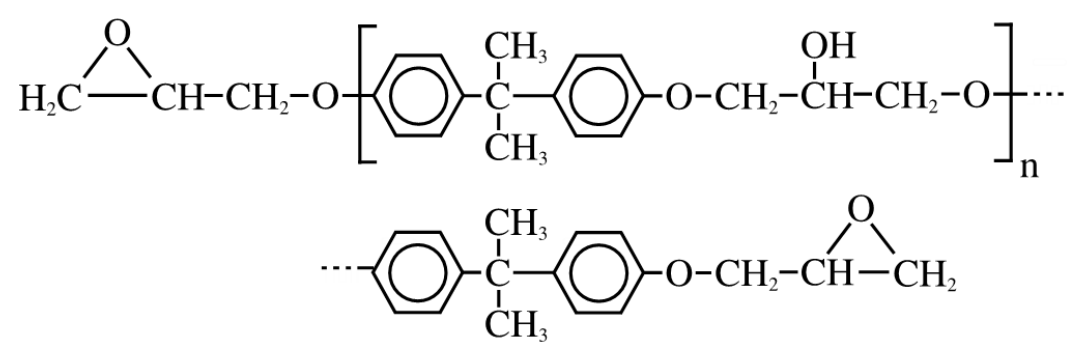

Figure 4.1: The molecular structure of EPON 828 resin [64].

The next materials that were tested were two variants of a bisphenol-A epoxy. Epoxies are thermosetting polymers that see widespread use in industry due to their favourable mechanical properties, which include high modulus and adhesion strength, low creep at elevated temperatures, and ease of application [63]. Epoxies are comprised of two parts: a resin and a curing agent. Mixing these two parts initiates a chemical reaction that yields a polymer with a highly-crosslinked epoxy network [64]. The kinetics of this reaction have a direct effect on the mechanical properties of the resulting epoxy, whereby variables such as cure temperature, mixing rate, stoichiometric ratio, and setting time all influence the polymerization process $[39,64]$. Recent developments in composite technology have led to a resurgence in research relating to the dynamic characterization of epoxies [6, 45, 65-69].

The epoxy systems that were chosen for experimentation comprised of EPON 828 resin cured with two different curing agents. EPON 828 is an undiluted, clear, difunctional, bisphenol-A liquid epoxy resin with an epoxy equivalent weight (EEW) of 185-192 g/mol. The structure of the base EPON 828 resin molecule is shown in Figure 4.1 [64]. The first curing agent used in this study was EPIKURE 3223, which is an unmodified aliphatic diethylenetriamine (DETA). The second curing agent was EPIKURE 3233, which is an unmodified aliphatic T-403 polyoxypropylenetriamine. A concise description of the physical meanings associated with the nomenclature of each compound is provided by Razack and Varghese [39]. All epoxy materials (curing agents and resin) were sourced from Hexion Inc. For convenience, the epoxy systems that resulted from curing EPON 828 resin with EPIKURE 3223 and EPIKURE 3233 will henceforth be referred to as EPON 828A and EPON 828-B, respectively. The bulk epoxy was made at the laboratory facilities of the 
National Research Council of Canada (NRC) by Meysam Rahmat. It was created by mixing the required quantities of the resin and curing agent with a planetary mixer (Thinky ARE 310) for two minutes. The EPON 828-A specimens comprised of a resin-to-curing agent mix ratio of 100:12 by weight, while the EPON 828-B specimens comprised of a resin-to-curing agent mix ratio of 100:43 by weight. The epoxy mixture was poured into open molds that were allowed to cure at room temperature for two days inside an autoclave with a minimum pressure of 85 psi. Once cured, the specimens were removed from their molds and then tempered at $60^{\circ} \mathrm{C}$ for two hours. After the post-cure tempering, the specimens were removed from the mold. Rough edges and excess material were removed by wet polishing with sandpaper.

The final material tested in the plate impact experiments was an epoxy/carbon nanotube composite. This material was fabricated by combining EPON 828-A epoxy (described in the previous paragraph) with pristine $\mathrm{NC} 7000^{\mathrm{TM}}$ industrial-grade multi-walled carbon nanotubes (MWCNT) supplied by Nanocyl SA. The nanotubes were produced via a catalytic chemical vapor deposition (CCVD) process. According to the manufacturer specifications, the nanotubes had a carbon purity of $90 \%$, an average length of $1.5 \mu \mathrm{m}$, an average diameter of $9.5 \mathrm{~nm}$, a density of $1.7 \mathrm{~g} \cdot \mathrm{cm}^{-3}$, and an average Brunauer-Emmett-Teller (BET) surface area of $250-300 \mathrm{~m}^{2} \cdot \mathrm{g}^{-1}$. This specific composite system was selected because of its suitability for acting as a stand-in for examining the effects of nano-scale inclusions on the shock response and dynamic failure behaviour of other, more sophisticated polymer-matrix composites. Results obtained from studying this epoxy/carbon nanotube composite could be used to optimize the microstructures of other nanocomposites with potential for genuine commercial application.

The bulk nanocomposite used in this study was fabricated at the laboratory facilities of the National Research Council of Canada (NRC) by Meysam Rahmat. Before adding the curing agent, a planetary mixer (Thinky ARE 310) was used to integrate the nanotubes into the resin. The required quantities of dry nanotubes and EPON 828 resin were combined and then mixed for three, three-minute cycles at $2000 \mathrm{rpm}$. After integration, the EPIKURE 3223 curing agent was added at a resin-to-curing agent weight ratio of 100:12 and mixed for two minutes. The same 
molding and curing procedures described earlier for preparing neat epoxy specimens was used for preparing nanocomposite specimens. The final nanocomposite contained $1 \mathrm{wt} \%$ pristine multiwalled carbon nanotubes.

\subsection{Load Frame Testing}

Tensile and compressive experiments were conducted using a Fullam Substage load frame to provide quasi-static data that would compliment the results of the plate impact experiments discussed in the following section. This testing allowed for the determination of several material properties, including tensile modulus $(E)$, yield strength $\left(\sigma_{y}\right)$, strain at yield stress $\left(\varepsilon_{y}\right)$, maximum tensile strength $\left(\sigma_{\max }\right)$, strain at maximum tensile stress $\left(\varepsilon_{\max }\right)$, strain energy density $\left(U_{t}\right)$, and fracture toughness $\left(K_{c}\right)$. The results of this work were analyzed in parallel with the results of the plate impact experiments to investigate failure at low and high strain rates (from $10^{-4}$ to $10^{4} \mathrm{~s}^{-1}$ ). All uniaxial tensile testing with the load frame was performed by Meysam Rahmat at the National Research Council Canada (NRC) in Ottawa. These tensile tests were conducted with dogbone specimens of each material according to ISO 527-2 (Type 1BB), where samples with a gauge

length of $12.0 \mathrm{~mm}$ were loaded at an extension rate of $2 \mathrm{~mm} \cdot \mathrm{min}^{-1}$. Testing was performed at $21^{\circ} \mathrm{C}$. The results of these uniaxial tensile tests were generously shared by Meysam Rahmat from the NRC. The complete set of results can be seen in Chapter 5, Table 5.1.

Plane-strain fracture toughness $\left(K_{C}\right)$ measurements were also taken for all material systems under quasi-static conditions. Fracture toughness defines the critical stress intensity of a material, above which brittle fracture will occur. Fracture toughness can be used to parameterize the ability of a material to resist critical crack propagation, which would result in total fracture. All fracture toughness testing with the load frame (set to compression) was performed by Behnam Ashrafi at the National Research Council Canada (NRC) in Ottawa. Rectangular specimens (2 mm x $4 \mathrm{~mm} \mathrm{x}$ $20 \mathrm{~mm}$ ) for each material were prepared in a Teflon ${ }^{\circledR}$ mould. A precision saw was used to create a notch on the samples. The notch was then sharpened with a razor blade. Preparation of the 


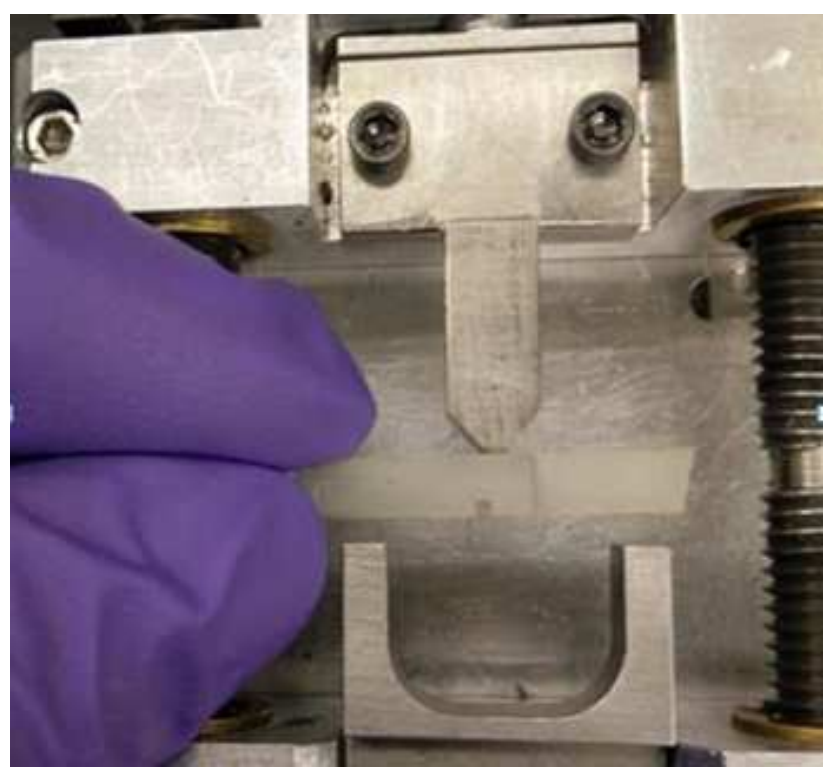

Figure 4.2: The load frame configured to compression for a fracture toughness test with a neat epoxy specimen. Photo courtesy of Behnam Ashrafi (NRC).

notches and testing were conducted according to ASTM D 5945 at $21^{\circ} \mathrm{C}$. The compression rate was $3 \mathrm{~mm} \cdot \mathrm{min}^{-1}$. A photo of the load frame used to conduct the fracture toughness tests is shown in Figure 4.2. The results of these fracture toughness tests were generously shared by Behnam Ashrafi from the NRC. The complete set of results can be seen in Chapter 5, Table 5.1.

\subsection{Plate Impact Experiments}

Three generalized experimental techniques have been devised for studying spall fracture in solids, each with their own virtues and shortcomings. They include: high-velocity plate impact, explosive shock-loading, and pulsed laser-deposition shock-loading [8]. For the present study, the highvelocity plate impact method was used. It is a tried-and-true technique of generating reliable experimental results, and was used in the present study due to the availability of the required facilities and equipment. The experimental setup used to conduct the plate impact experiments, which consisted of a single-stage light gas gun, a photonic-Doppler velocimeter, and a data acquisition system, is discussed in detail below. 
Table 4.1: Acoustic properties of all relevant materials, where $\rho_{0}$ is bulk density, $C_{0}$ is bulk sound speed, $C_{L}$ is longitudinal sound speed, and $Z$ is shock impedance.

\begin{tabular}{lcccc}
\hline Material & $\begin{array}{c}\rho_{0} \\
\left(\mathrm{~g} \cdot \mathrm{cm}^{-3}\right)\end{array}$ & $\begin{array}{c}C_{0} \\
\left(\mathrm{~km} \cdot \mathrm{s}^{-1}\right)\end{array}$ & $\begin{array}{c}C_{L} \\
\left(\mathrm{~km} \cdot \mathrm{s}^{-1}\right)\end{array}$ & $\begin{array}{c}Z \\
\left(\mathrm{GPa} \cdot \mathrm{s} \cdot \mathrm{m}^{-1}\right)\end{array}$ \\
\hline 6061-T6 & $2.70^{\mathrm{a}}$ & $5.35^{\mathrm{c}}$ & $6.40^{\mathrm{c}}$ & 14.44 \\
PMMA & $1.193^{\mathrm{b}}$ & $2.60^{\mathrm{c}}$ & $2.72^{\mathrm{c}}$ & 3.08 \\
EPON 828-A & $1.185^{\mathrm{c}}$ & $2.64^{\mathrm{c}}$ & $2.63^{\mathrm{c}}$ & 3.13 \\
EPON 828-A + P-CNT & $1.185^{\mathrm{c}}$ & $2.64^{\mathrm{c}}$ & $2.63^{\mathrm{c}}$ & 3.13 \\
EPON 828-B & $1.185^{\mathrm{c}}$ & $2.64^{\mathrm{c}}$ & $2.63^{\mathrm{c}}$ & 3.13 \\
\hline
\end{tabular}

${ }^{a}$ Glemco Inc. [70]

${ }^{\mathrm{b}}$ Arkema Inc. [61]

${ }^{\mathrm{c}}$ Carter and Marsh [33]

A summary of the acoustic properties of the materials tested in the plate impact experiments are listed in Table 4.1. It is vital to note that the values of $C_{L}$ and $C_{0}$ reported in this table were not obtained from our own ultrasonic (pulse-echo transit) measurements, but rather, were taken directly from the shock Hugoniot data for EPON 828 presented by Carter and Marsh [33]. These sound speeds listed for EPON 828-A and EPON 828-B will be assumed to be independent of the curing agent used to set the resin, and therefore valid for calculations of spall strength. It was also assumed that the addition of $1 \mathrm{wt}$ \% carbon nanotubes (CNT) to EPON 828-A had a negligible effect on the bulk and longitudinal sound speeds of the epoxy system.

\subsubsection{Gas Gun}

A single-stage light gas gun with a $64 \mathrm{~mm}$ smooth internal bore was used for the plate impact experiments. Access to the gas gun laboratory, located in Montréal, QC, at McGill University was granted by Andrew Higgins. A schematic of the gas gun is shown in Figure 4.3. The device operated by accelerating a projectile along a barrel using pressure imparted by the rapid expansion of compressed gas (typically air, nitrogen, or helium). The gas gun achieved projectile impact velocities between 118 and $722 \mathrm{~m} \cdot \mathrm{s}^{-1}$. Prior to launching the projectile, the catch tank was evacuated to a pressure of approximately $150 \mathrm{~Pa}$. This helped minimize the aerodynamic drag acting on the projectile, thereby allowing higher impact velocities to be achieved. The projectiles comprised of a plastic (nylon or PVC) sabot fitted with a flyer plate made from either aluminum 6061-T6 or 


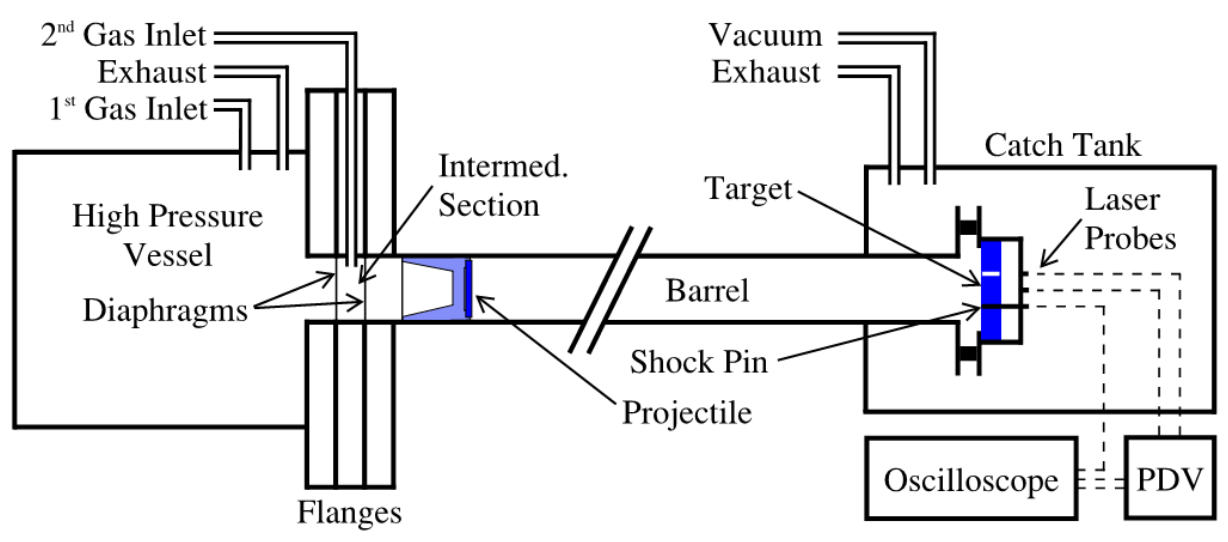

Figure 4.3: A schematic of the single-stage light gas gun.

PMMA. The aluminum and PMMA flyer plates $(60 \mathrm{~mm}$ diameter) were laser cut from $1.96 \mathrm{~mm}$ and $1.55 \mathrm{~mm}$ sheet stock, respectively. A relief port was drilled through the wall of the sabot into an air gap located directly behind the flyer plate. The purpose of this relief port was to allow the gas pressure on both sides of the flyer plate to equilibrate during the evacuation of the catch tank, which helped to eliminate any undesired flexing/loading. The material specimens, commonly referred to as targets, were fixed to the target mount. The target mount was secured to the muzzle of the gas gun barrel to help ensure planarity between the flyer plate and target during impact.

\subsubsection{Target Fabrication}

The aluminum 6061-T6 targets used in the plate impact experiments were machined into discs from cold-rolled plate stock. The plate stock was sourced from local metal retailers. The surfaces of the targets were were not polished after machining Chapter 5, Table 5.2. Aluminum is infraredreflective, so it was not necessary to introduce a buffer material to the back surface of each target to reflect the infrared laser emitted the photonic Doppler velocimeter (discussed in the following section). A photo of a fully-instrumented aluminum 6061-T6 target set up in the target mount is shown in Figure 4.4. Results from the plate impact experiments with aluminum 6061-T6 are presented in Chapter 5, section 5.1.

The PMMA targets were machined from $9.53 \mathrm{~mm}$ (3/8 inch) sheet stock of Plexiglas ${ }^{\circledR} \mathrm{G}$ [61]. Only one side of the discs were faced in order to preserve the quality of the factory finish. The final 


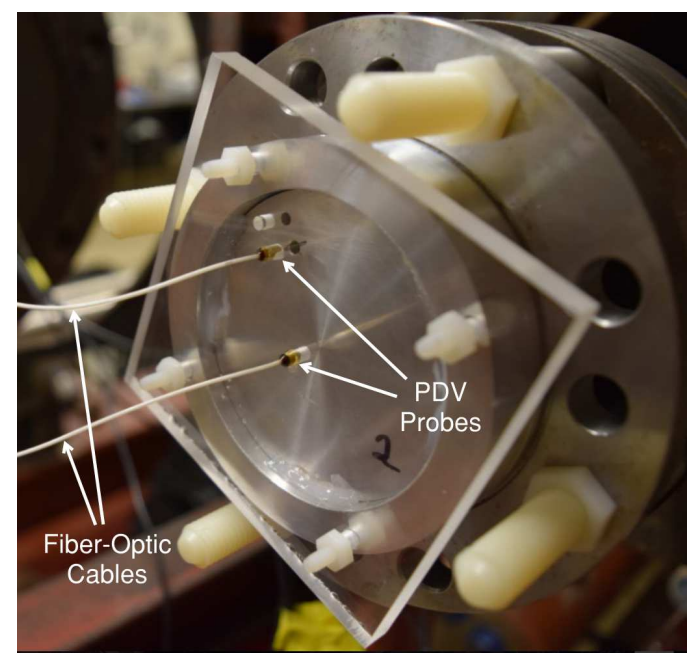

Figure 4.4: The target mount with a fullyinstrumented aluminum 6061-T6 target.

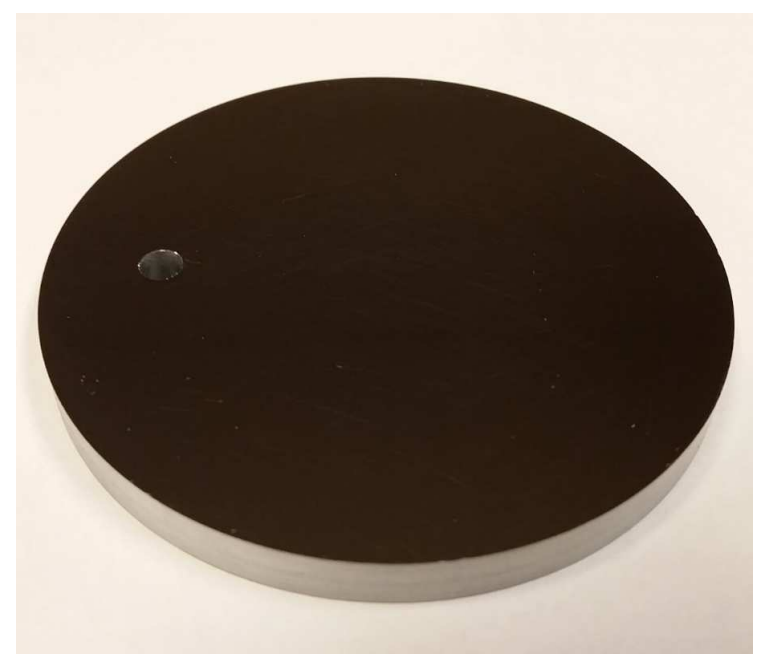

Figure 4.5: The sputter-coated side of a PMMA target showing a mirror finish.

thicknesses of each target $\left(w_{t}\right)$ are reported in Chapter 5, Table 5.2. The not-machined side of the targets were subjected to the aluminum sputter-coating process described in the following section. A photo of a typical sputter-coated PMMA target is shown in Figure 4.5. Results from the plate impact experiments with PMMA are presented in Chapter 5, section 5.2.

The neat epoxy targets were machined from molded plates (made using the process described in section 4.1). The surfaces of the EPON 828-A targets were not polished after machining (i.e., kept in their as-machined state), whereas the front and back surfaces of the EPON 828-B targets were polished with a stepwise process that used up to 400 grit sandpaper to remove any visible surface imperfections. The thicknesses of the targets $\left(w_{t}\right)$ used in the experiments are listed in Chapter 5, Table 5.2. Results from the plate impact experiments with EPON 828-A and EPON 828-B targets are presented in Chapter 5, section 5.3.

The nanocomposite targets were also machined from molded plates (made using the process described in section 4.1). The front and back surfaces of the targets were polished with a stepwise process that used up to 400 grit sandpaper to remove any visible surface imperfections. The thicknesses of the targets $\left(w_{t}\right)$ used in the experiments are listed in Chapter 5, Table 5.2.

A small square of 0.002 inch thick (200 gauge) aluminized Mylar® foil was super-glued to the back surface of most of the nanocomposite targets. This was done to reflect the infrared laser 


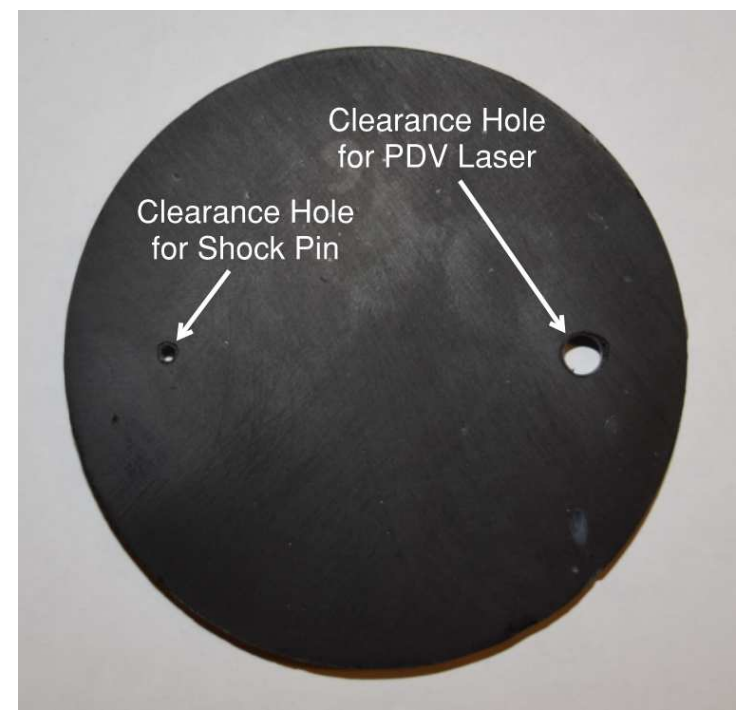

Figure 4.6: An EPON 828-A + P-CNT target for use in the plate impact experiments.

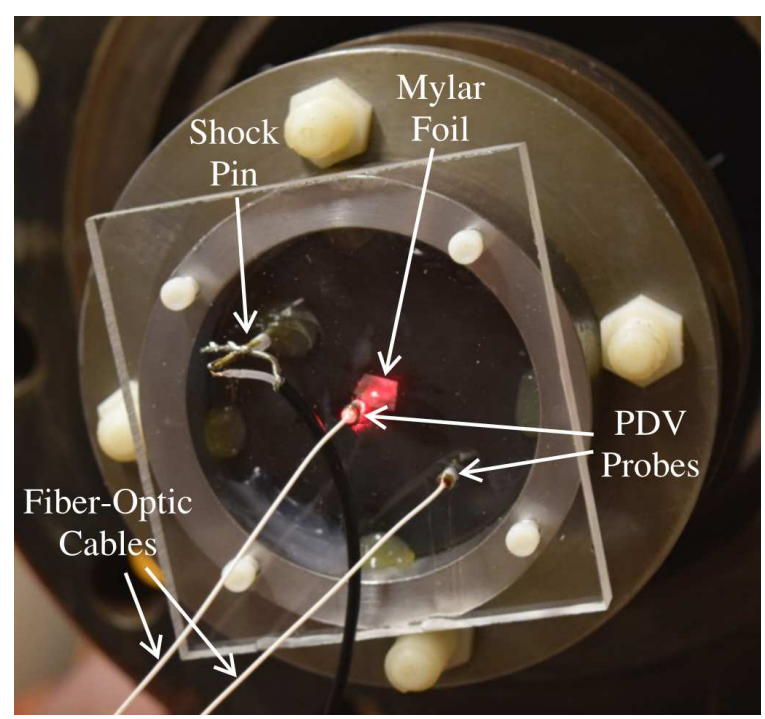

Figure 4.7: The target mount with a fullyinstrumented EPON 828-A + P-CNT target.

emitted the photonic Doppler velocimeter (discussed in detail the following section). Mylar® is a DuPont® polyester (PET) film. Results from the plate impact experiments with nanocomposite targets are presented in Chapter 5, section 5.4. A photo of a typical nanocomposite target used in the impact experiments is shown in Figure 4.6. A photo of a fully-instrumented nanocomposite target is shown in Figure 4.7.

\subsubsection{Target Instrumentation}

The targets were instrumented with a piezoelectric (shock) pin and laser collimators connected to a two-channel photonic-Doppler velocimeter (PDV) via fiber optic cables. A description of compact PDV systems is provided by Strand et al. [71]. A schematic of the target assembly used in the plate impact experiments is provided in Figure 4.8. Note that an alternate assembly was used that did not instrument the targets with a shock pin. A photo of this alternate assembly is shown in Figure 4.9.

The shock pins (model CA-1135) were purchased from Dynasen Inc. They were mounted flush with the front face of the target. Upon impact, the piezoelectric crystal on the tip of the pin was compressed, which generated a voltage signal that was registered by the oscilloscope. The instant 


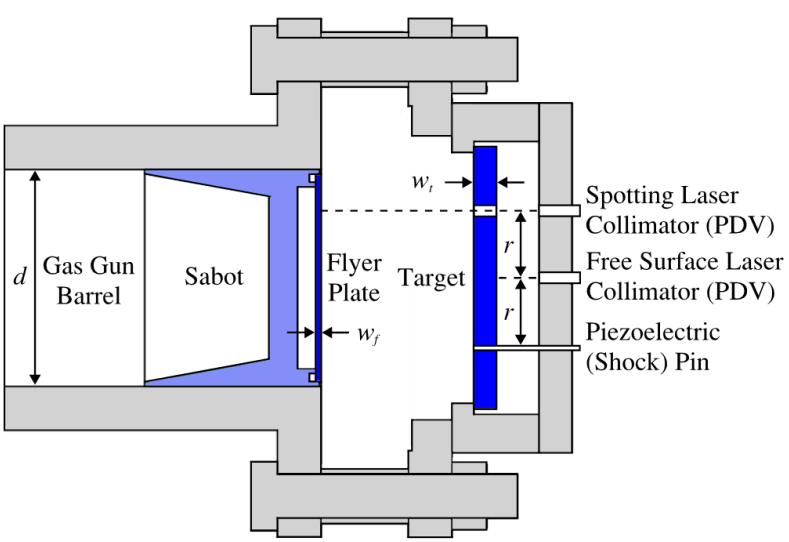

Figure 4.8: A schematic of the target assembly, where $d=64 \mathrm{~mm}, r=25.4 \mathrm{~mm}, w_{f}=1.96$ or $1.55 \mathrm{~mm}$, and $w_{t}=3.02 \sim 6.60 \mathrm{~mm}$.

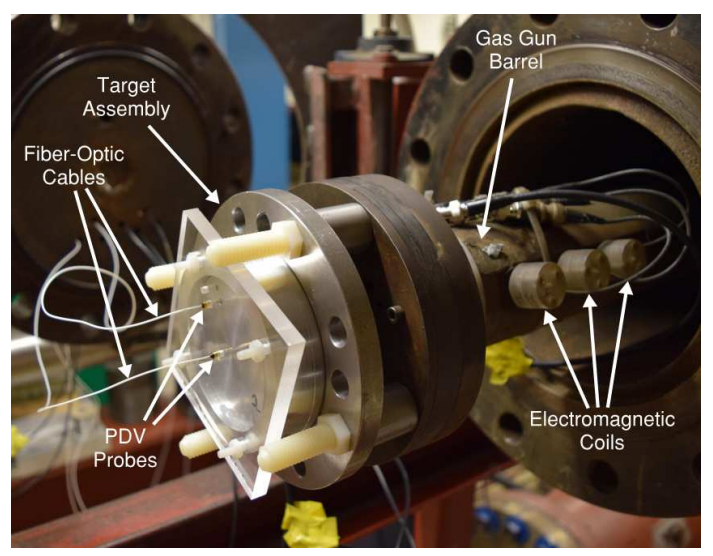

Figure 4.9: A labeled photo of the target assembly. This particular setup did not include a shock pin.

of initial compression marked the time of impact (TOI) of the flyer plate against the target. The TOI was used with information from the PDV to determine the shock transit time in the target.

The PDV was used to simultaneously measure the velocity of the flyer plate and the back surface of the target with high temporal resolution. To generalize, a PDV operates by projecting a colimated laser at a reflective surface [71]. When the surface moves, the frequency of the reflected light is altered via a process known as Doppler-shifting [71]. The reflected light is then recombined with the reference laser light in the detector. Since the reflected light has a different frequency than the reference light, combining them will produce a beat frequency [71]. If the surface accelerates, then the beat frequency will change. Therefore fluctuations in the beat frequency can be linked to accelerations of the surface, while specific beat frequencies can be linked to specific velocities. A schematic that illustrates the fundamental components of a PDV is shown in Figure 4.10.

As mentioned earlier, two separate collimators were used (see Figure 4.8). One collimator projected the laser on the back surface of the target, while the other projected the laser at the flyer plate (through a clearance hole in the target). The laser directed at the flyer plate (referred to as the spotting laser) was used to measure the velocity of the projectile immediately before impact.

A secondary method for measuring impact velocity was also employed during the early stages of testing. This method utilized a series electromagnetic coil gauges embedded at $40 \mathrm{~mm}$ intervals $\left(x_{\text {coil }}\right)$ along the gas gun barrel (at the muzzle). A magnet was mounted on each projectile. As a 


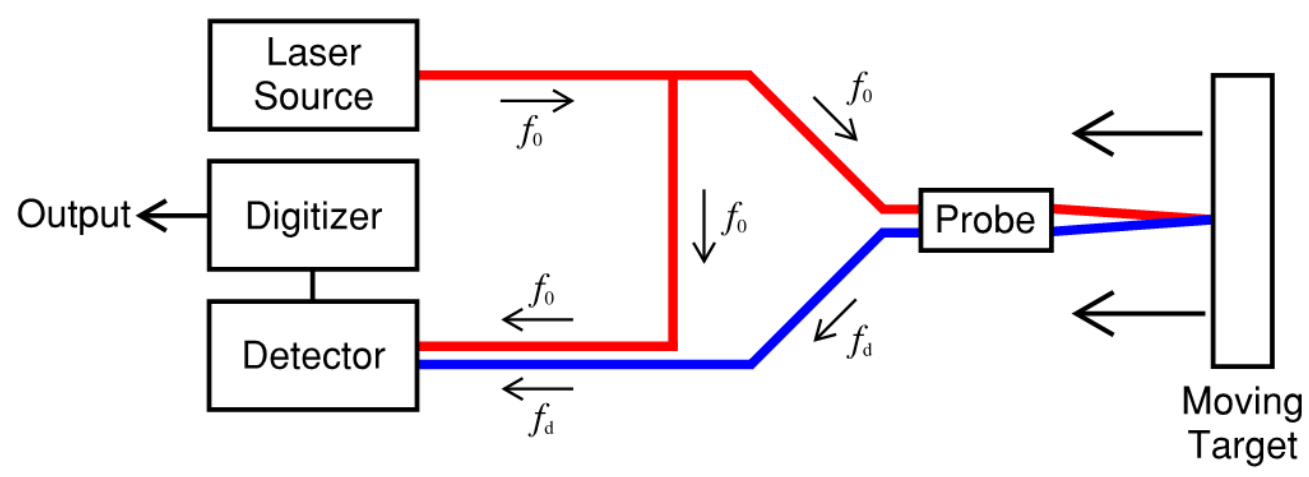

Figure 4.10: A schematic of a PDV system that depicts how the reference light (with frequency $f_{0}$ ) and the reflected light (with frequency $f_{d}$ ) are recombined at the detector [71].

projectile translated along the barrel, the magnet would generate a current in the electromagnetic coils in sequential order. A plot of the voltage signals produced by the coils can be seen in Figure 4.11. Each signal has a distinct voltage spike that is associated with the arrival of the projectile. The timing of each voltage spike $(t)$ was recorded, which allowed for the impact velocity of the projectile $\left(v_{i}\right)$ to be determined using

$$
v_{i}=\frac{1}{2}\left(\frac{x_{\text {coil }}}{t_{2}-t_{1}}+\frac{x_{\text {coil }}}{t_{3}-t_{2}}\right) .
$$

The specific PDV system used in the present study had been validated previously by Professor Oren Petel. Surface velocities measured with the PDV system were found to be in close agreement with velocities measured using the coil gauge technique described earlier, with an associated error of $\pm 10 \mathrm{~m} \cdot \mathrm{s}^{-1}$. The PDV system used a $1550 \mathrm{~nm}$ infrared laser. In order for the PDV to obtain accurate measurements, the target material had to be infrared reflective. Unfortunately, the polymers that were tested in the present study were not infrared-reflective. This made it impossible to take direct measurements of the free surface velocity during impact. For these materials it was necessary to introduce a reflective buffer to the back face of each target. This was achieved with two different methods. The first method involved using a semicore inline sputtering system to coat the back surface with a 40-60 nm layer of aluminum. This required the samples to be held in a vacuum chamber for several hours to allow for sufficient degassing of the material, followed by the coating process, which occurred at $50^{\circ} \mathrm{C}$. It was found that the quality of the coating improved 


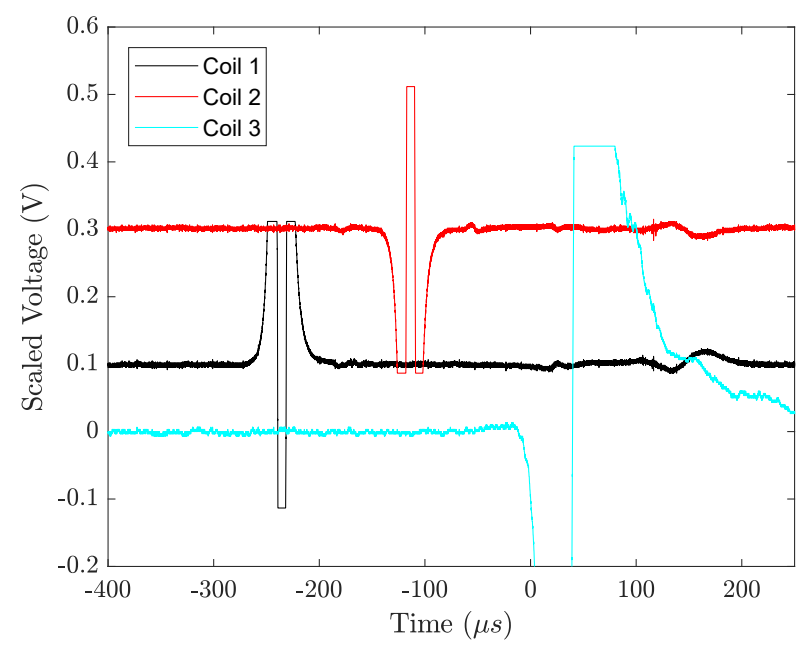

Figure 4.11: An example of the voltage signals obtained from the electromagnetic coils during a shot with the gas gun.

if the samples were polished prior to subjecting them to the sputtering system. The second method involved adhering a small square of 0.002 inch thick aluminized Mylar® foil to the back face of the target with superglue. This method was found to generate spectrographic velocity histories with improved spatial resolution when compared to signals obtained using the aluminum sputter-coating method, with the added benefit of being more cost-efficient.

\subsubsection{Signal Processing}

The shock pin and PDV signals were recorded using an oscilloscope at a sampling rate of 40 GS $\cdot \mathrm{s}^{-1}$, which was sufficient to yield a temporal resolution that allowed for the observation of spall phenomena $[31,71]$. A temporal deflection of $200 \mathrm{mV} \cdot \mathrm{div}^{-1}$ was used. The oscilloscope deflection for the PDV signals was set to $500 \mathrm{mV} \cdot \mathrm{div}^{-1}$. A typical shock pin signal is shown in Figure 4.12(a). An example of a waveform retrieved from the raw PDV data is shown in Figure 4.12(b). The waveforms obtained by the oscilloscope were processed with a windowed Fourier transform that returned a spectrogram showing a distribution of laser beat frequencies, where the dominant frequency was associated with the motion of the target [71]. The procedure described in section 3.4 can be used to determine the dynamic mechanical properties of the target material from 
the analysis of the free surface velocity profiles observed on the spectrograms. The spectrogram that corresponds to the waveform presented in Figure 4.12(b) is shown in Figure 4.12(c). The Matlab scripts used to process the PDV data, shock pin data, and coil gauge data are provided in Appendix A. 


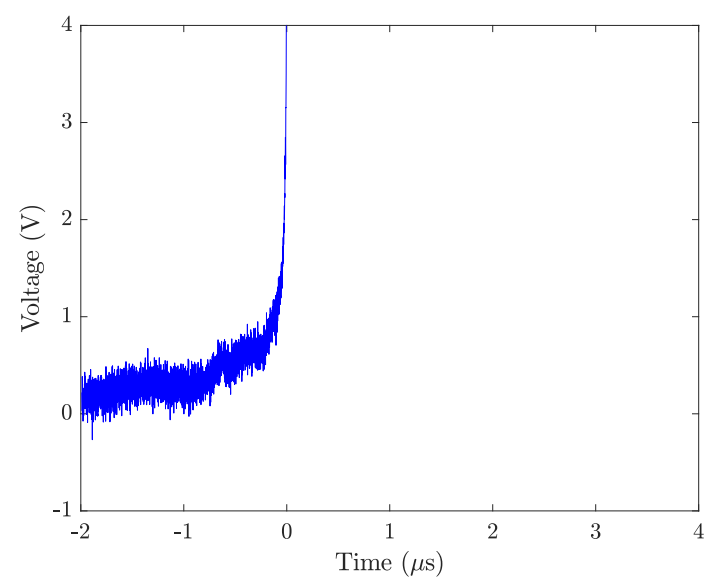

(a)

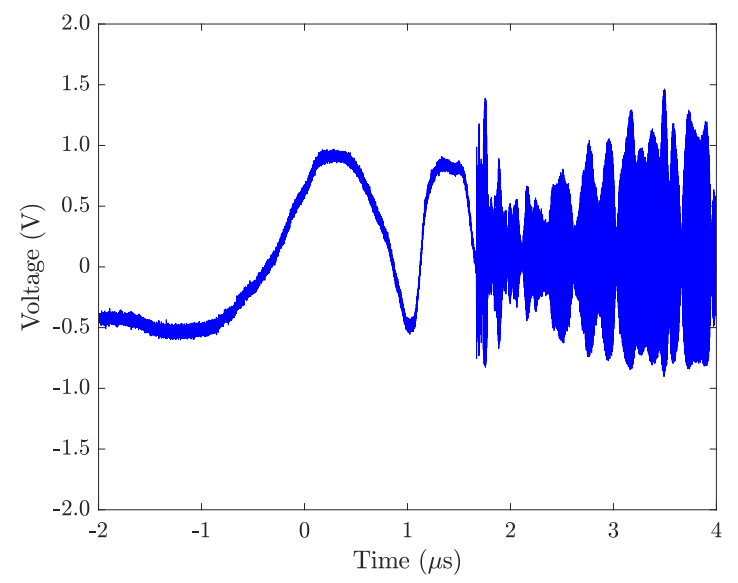

(b)

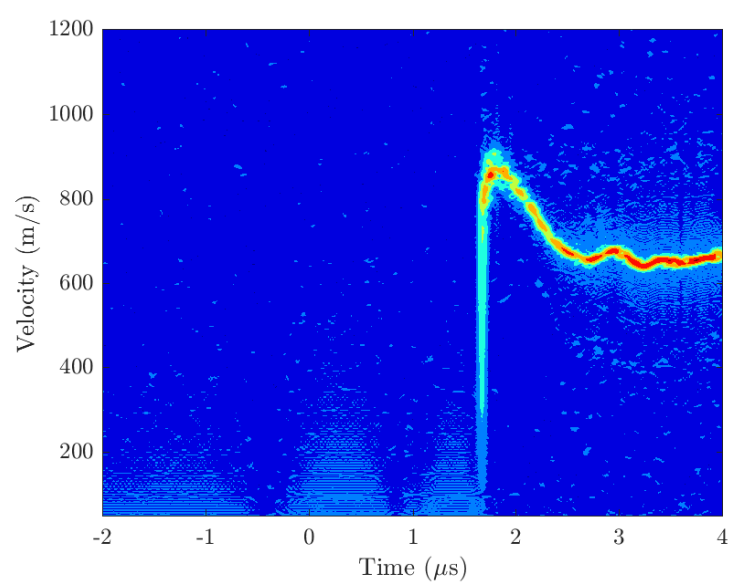

(c)

Figure 4.12: (a) The shock pin signal of an EPON 828 / EPIKURE 3223 epoxy + 1 wt.\% PCNT target impacted with an aluminum flyer plate at $617 \mathrm{~m} \cdot \mathrm{s}^{-1}$. (b) The waveform generated by the PDV system for the aforementioned impact, and (c) its associated spectrogram. 


\section{Chapter 5}

\section{Results and Discussion}

This section contains the complete set of results from the load frame testing (Table 5.1) and the plate impact testing (Table 5.2). The

results are presented in tabulated form to facilitate cross-referencing. The results from each experiment will now be discussed on a material-by-material basis in the following sections of this report.

Table 5.1: The results of the quasi-static uniaxial tensile experiments and the quasi-static fracture toughness experiments conducted by Meysam Rahmat and Behnam Ashrafi, respectively. STD refers to the standard deviation.

\begin{tabular}{|c|c|c|c|c|c|c|c|c|c|c|c|c|c|c|c|}
\hline Material & $\begin{array}{c}\text { Date } \\
(\mathrm{mm} / \mathrm{yy})\end{array}$ & $\begin{array}{c}E \\
(\mathrm{GPa})\end{array}$ & $\begin{array}{c}\text { Std } \\
(\mathrm{MPa})\end{array}$ & $\begin{array}{c}\sigma_{y} \\
(\mathrm{MPa})\end{array}$ & $\begin{array}{c}\text { Std } \\
(\mathrm{MPa})\end{array}$ & $\varepsilon_{y}$ & Std & $\begin{array}{c}\sigma_{\max } \\
(\mathrm{MPa})\end{array}$ & $\begin{array}{c}\text { Std } \\
(\mathrm{MPa})\end{array}$ & $\varepsilon_{\max }$ & Std & $\begin{array}{c}U_{t} \\
\mathrm{~J} \cdot \mathrm{m}^{-3}\end{array}$ & $\underset{\mathrm{J} \cdot \mathrm{m}^{-3}}{\mathrm{Std}}$ & $\begin{array}{c}K_{c} \\
\left(\mathrm{MPa} \cdot \mathrm{m}^{1 / 2}\right)\end{array}$ & $\begin{array}{c}\mathrm{Std} \\
\left(\mathrm{MPa} \cdot \mathrm{m}^{1 / 2}\right)\end{array}$ \\
\hline 6061-T6 & - & $68.9^{\mathrm{a}}$ & - & $276^{\mathrm{a}}$ & - & - & - & $310^{\mathrm{a}}$ & - & $0.120^{\mathrm{a}}$ & - & - & - & $29^{\mathrm{a}}$ & - \\
\hline PMMA & - & $3.10^{\mathrm{b}}$ & - & $72.4^{\mathrm{b}}$ & - & - & - & $72.4^{\mathrm{b}}$ & - & $0.050^{\mathrm{b}}$ & - & - & - & $1.55^{\mathrm{c}}$ & - \\
\hline EPON 828-A & Old & 1.93 & 21 & 25.4 & 2.8 & 0.015 & 0.002 & 69.1 & 3.7 & 0.069 & 0.016 & 3.10 & 1.10 & 1.00 & 0.08 \\
\hline EPON 828-A & $08 / 16$ & 1.94 & 91 & 27.7 & 2.4 & 0.016 & 0.002 & 49.1 & 3.7 & 0.047 & 0.008 & 1.52 & 0.42 & 1.05 & 0.20 \\
\hline EPON 828-A & $03 / 17$ & 2.65 & 95 & 29.7 & 5.8 & 0.013 & 0.002 & 65.0 & 2.5 & 0.046 & 0.007 & 1.91 & 0.45 & 1.02 & 0.14 \\
\hline EPON 828-B & 03/17 & 2.85 & 81 & 38.1 & 10.0 & 0.015 & 0.003 & 58.6 & 1.5 & 0.037 & 0.001 & 1.88 & 0.15 & 2.07 & 0.24 \\
\hline EPON 828-A + P-CNT & $08 / 16$ & 1.87 & 39 & 27.3 & 2.6 & 0.017 & 0.001 & 44.1 & 13.3 & 0.035 & 0.015 & 1.00 & 0.62 & 1.34 & 0.05 \\
\hline
\end{tabular}


Table 5.2: The results of the plate impact experiments.

\begin{tabular}{|c|c|c|c|c|c|c|c|c|c|c|c|c|c|c|c|}
\hline Shot \# & Target & $\begin{array}{c}w_{t} \\
(\mathrm{~mm})\end{array}$ & Flyer Plate & $\begin{array}{c}w_{f} \\
(\mathrm{~mm})\end{array}$ & $\begin{array}{c}v_{i} \\
\left(\mathrm{~m} \cdot \mathrm{s}^{-1}\right)\end{array}$ & $\begin{array}{c}u_{\text {peak }} \\
\left(\mathrm{m} \cdot \mathrm{s}^{-1}\right)\end{array}$ & $\begin{array}{c}u_{\text {pull }} \\
\left(\mathrm{m} \cdot \mathrm{s}^{-1}\right)\end{array}$ & $\begin{array}{c}\sigma_{s p} \\
(\mathrm{MPa})\end{array}$ & $\begin{array}{c}\mathrm{d} u_{f s}(t) / \mathrm{d} t \\
\left(\mathrm{~m} \cdot \mathrm{s}^{-2}\right)\end{array}$ & $\begin{array}{c}\mathcal{E}_{r} \\
\left(\mathrm{~s}^{-1}\right)\end{array}$ & $\begin{array}{c}K_{c} \\
\left(\mathrm{MPa} \cdot \mathrm{m}^{1 / 2}\right)\end{array}$ & $\begin{array}{c}u_{p} \\
\left(\mathrm{~km} \cdot \mathrm{s}^{-1}\right)\end{array}$ & $\begin{array}{c}U_{s} \\
\left(\mathrm{~km} \cdot \mathrm{s}^{-1}\right)\end{array}$ & $\begin{array}{c}\sigma_{H} \\
(\mathrm{GPa})\end{array}$ & $\varepsilon_{H}$ \\
\hline 1 & 6061-T6 & 9.35 & 6061-T6 & 1.96 & 580 & 550 & 354 & 1542 & $-4.89 \times 10^{8}$ & $4.57 \times 10^{4}$ & 43.0 & 0.28 & $5.72^{\mathrm{a}}$ & $4.25^{\mathrm{a}}$ & $0.05^{\mathrm{a}}$ \\
\hline 2 & 6061-T6 & 9.47 & 6061-T6 & 1.96 & 633 & 633 & 463 & 1338 & $-4.80 \times 10^{8}$ & $4.49 \times 10^{4}$ & 35.1 & 0.32 & 4.76 & 4.07 & 0.07 \\
\hline 3 & 6061-T6 & 9.71 & 6061-T6 & 1.96 & 667 & 656 & 463 & 1519 & $-4.16 \times 10^{8}$ & $3.89 \times 10^{4}$ & 45.6 & 0.33 & $5.79^{\mathrm{a}}$ & $5.13^{\mathrm{a}}$ & $0.06^{\mathrm{a}}$ \\
\hline 4 & PMMA & 6.55 & 6061-T6 & 1.96 & 208 & 328 & 231 & 153 & $-4.13 \times 10^{8}$ & $7.95 \times 10^{4}$ & 2.21 & 0.16 & 2.86 & 0.56 & 0.06 \\
\hline 5 & PMMA & 6.53 & 6061-T6 & 1.96 & 502 & 703 & 589 & 180 & $-3.10 \times 10^{8}$ & $5.96 \times 10^{4}$ & 3.24 & 0.35 & 3.52 & 1.47 & 0.10 \\
\hline 6 & PMMA & 8.26 & 6061-T6 & 1.96 & 606 & 774 & 668 & 168 & $-2.65 \times 10^{8}$ & $5.09 \times 10^{4}$ & 3.16 & 0.39 & 3.28 & 1.51 & 0.12 \\
\hline 7 & PMMA & 8.45 & 6061-T6 & 1.96 & 656 & 812 & 686 & 200 & $-8.21 \times 10^{8}$ & $1.58 \times 10^{5}$ & 2.33 & 0.41 & 3.29 & 1.59 & 0.12 \\
\hline 8 & PMMA & 6.55 & 6061-T6 & 1.96 & 722 & 1010 & 895 & 181 & $-3.86 \times 10^{8}$ & $7.43 \times 10^{4}$ & 2.94 & 0.51 & 3.33 & 2.00 & 0.15 \\
\hline 9 & PMMA & 6.55 & PMMA & 1.55 & 203 & 3188 & 3084 & 164 & $-1.99 \times 10^{8}$ & $3.83 \times 10^{4}$ & 3.53 & 0.10 & $2.98^{\mathrm{b}}$ & $0.35^{\mathrm{b}}$ & $0.03^{b}$ \\
\hline 10 & PMMA & 6.55 & PMMA & 1.55 & 252 & 3216 & 3112 & 164 & $-1.76 \times 10^{8}$ & $3.39 \times 10^{4}$ & 3.75 & 0.11 & $3.00^{\mathrm{b}}$ & $0.38^{\mathrm{b}}$ & $0.04^{\mathrm{b}}$ \\
\hline 11 & PMMA & 6.60 & PMMA & 1.55 & 357 & 3385 & 3285 & 158 & $-5.36 \times 10^{8}$ & $1.03 \times 10^{5}$ & 2.03 & 0.18 & $3.12^{\mathrm{b}}$ & $0.66^{\mathrm{b}}$ & $0.06^{\mathrm{b}}$ \\
\hline 12 & PMMA & 6.50 & PMMA & 1.55 & 498 & 498 & 398 & 158 & $-1.77 \times 10^{8}$ & $3.40 \times 10^{4}$ & 3.53 & 0.25 & $3.20^{\mathrm{b}}$ & $0.95^{\mathrm{b}}$ & $0.08^{b}$ \\
\hline 13 & PMMA & 6.53 & PMMA & 1.55 & 563 & 3644 & 3443 & 159 & $-2.88 \times 10^{8}$ & $5.54 \times 10^{4}$ & 2.81 & 0.28 & $3.22^{\mathrm{b}}$ & $1.06^{\mathrm{b}}$ & $0.09^{\mathrm{b}}$ \\
\hline 14 & EPON 828-A & 6.50 & 6061-T6 & 1.96 & 219 & 345 & 86 & 404 & $-1.70 \times 10^{8}$ & $3.22 \times 10^{4}$ & 14.8 & 0.17 & 2.77 & 0.57 & 0.06 \\
\hline 15 & EPON 828-A & 5.38 & 6061-T6 & 1.96 & 363 & 561 & 276 & 445 & $-2.11 \times 10^{8}$ & $4.00 \times 10^{4}$ & 15.3 & 0.28 & 2.79 & 0.93 & 0.10 \\
\hline 16 & EPON 828-A & 6.58 & 6061-T6 & 1.96 & 503 & 771 & 435 & 525 & $-2.39 \times 10^{8}$ & $4.53 \times 10^{4}$ & 18.4 & 0.39 & 3.11 & 1.42 & 0.12 \\
\hline 17 & EPON 828-A & 6.43 & 6061-T6 & 1.96 & 671 & 1023 & 657 & 571 & $-2.92 \times 10^{8}$ & $5.53 \times 10^{4}$ & 19.0 & 0.51 & 3.32 & 2.01 & 0.15 \\
\hline 18 & EPON 828-A & 6.27 & 6061-T6 & 1.96 & 715 & 1109 & 734 & 585 & $-4.03 \times 10^{8}$ & $7.64 \times 10^{4}$ & 16.7 & 0.56 & 3.38 & 2.22 & 0.16 \\
\hline 19 & EPON 828-A & 5.44 & PMMA & 1.55 & 136 & 3113 & 2984 & N.S. ${ }^{c}$ & - & - & - & 0.07 & - & - & - \\
\hline 20 & EPON 828-A & 6.50 & PMMA & 1.55 & 585 & 605 & 465 & S.I. ${ }^{\mathrm{d}}$ & - & - & - & 0.30 & - & - & - \\
\hline 21 & EPON 828-B & 6.58 & 6061-T6 & 1.96 & 118 & 193 & 0 & N.S. ${ }^{c}$ & - & - & - & 0.10 & 2.38 & 0.27 & 0.04 \\
\hline 22 & EPON 828-B & 6.58 & 6061-T6 & 1.96 & 318 & 450 & 233 & 339 & $-1.63 \times 10^{8}$ & $3.08 \times 10^{4}$ & 11.6 & 0.23 & 2.72 & 0.73 & 0.08 \\
\hline 23 & EPON 828-B & 6.60 & 6061-T6 & 1.96 & 471 & 697 & 443 & 397 & $-2.94 \times 10^{8}$ & $5.57 \times 10^{4}$ & 10.9 & 0.35 & 3.43 & 1.42 & 0.10 \\
\hline 24 & EPON 828-B & 6.68 & PMMA & 1.55 & 344 & 350 & 133 & 339 & $-2.73 \times 10^{8}$ & $5.17 \times 10^{4}$ & 8.95 & 0.18 & 2.87 & 0.60 & 0.06 \\
\hline 25 & EPON 828-B & 6.60 & PMMA & 1.55 & 484 & - & - & S.I. ${ }^{\mathrm{d}}$ & - & - & - & - & - & - & - \\
\hline 26 & EPON 828-B & 6.53 & PMMA & 1.55 & 638 & 685 & 434 & 392 & $-3.70 \times 10^{8}$ & $7.00 \times 10^{4}$ & 9.57 & 0.34 & $3.17^{\mathrm{e}}$ & $1.29^{\mathrm{e}}$ & $0.11^{\mathrm{e}}$ \\
\hline 27 & EPON 828-A + P-CNT & 5.08 & 6061-T6 & 1.96 & 617 & 873 & 655 & 325 & $-3.73 \times 10^{8}$ & $7.06 \times 10^{4}$ & 7.23 & 0.23 & 3.70 & 0.98 & 0.06 \\
\hline 28 & EPON 828-A + P-CNT & 4.37 & PMMA & 1.55 & 346 & 356 & 135 & 342 & - & - & - & 0.18 & 2.67 & 0.56 & 0.07 \\
\hline 29 & EPON 828-A + P-CNT & 3.02 & PMMA & 1.55 & 353 & 349 & 161 & 291 & $-3.95 \times 10^{8}$ & $7.48 \times 10^{4}$ & 5.95 & 0.18 & 1.96 & 0.40 & 0.09 \\
\hline 30 & EPON 828-A + P-CNT & 3.07 & PMMA & 1.55 & 483 & 473 & 400 & 113 & - & - & - & 0.24 & 1.82 & 0.51 & 0.13 \\
\hline 31 & EPON 828-A + P-CNT & 4.57 & PMMA & 1.55 & 499 & 495 & 290 & 317 & $-3.57 \times 10^{8}$ & $6.76 \times 10^{4}$ & 7.13 & 0.25 & 1.62 & 0.47 & 0.15 \\
\hline 32 & EPON 828-A + P-CNT & 5.21 & PMMA & 1.55 & 625 & 585 & 367 & 337 & $-3.63 \times 10^{8}$ & $6.88 \times 10^{4}$ & 7.75 & 0.29 & 5.04 & 1.73 & 0.06 \\
\hline
\end{tabular}

${ }^{\mathrm{a}} U_{s}$ was calculated using the empirical polynomial $U_{s}=1.34 u_{p}+5.35$, a polynomial fitted to experimental data Marsh [72].

${ }^{\mathrm{b}} U_{s}$ was calculated using the empirical polynomial $U_{s}=6.486 u_{p}^{3}-7.823 u_{p}^{2}+3.549 u_{p}+2.703$ provided by Jordan et al. for $u_{p} \leq 0.4 \mathrm{~km} \cdot \mathrm{s}^{-1}[32]$.

c N.S. refers to a "no-spall" impact, where the characteristic pullback signal was not observed on the velocity profile.

d S.I. refers to a "scattered impact", where the flyer plate is presumed to have fractured prior to striking the target.

${ }^{\mathrm{e}} U_{s}$ was calculated using the empirical polynomial $U_{s}=0.848 u_{p}^{3}-2.221 u_{p}^{2}+3.287 u_{p}+2.275$, a polynomial fitted to experimental data by Carter and Marsh for $u_{p} \leq 1.0 \mathrm{~km} \cdot \mathrm{s}^{-1}$ [33]. 


\subsection{Aluminum 6061-T6}

The experimental setup used for the plate impact experiments with aluminum 6061-T6 targets did not include shock pins. This meant that the time of impact (TOI) of the flyer plate was not identified, making it impossible to accurately determine shock transit times in the specimens. Fortunately, the Hugoniot state particle velocities $\left(u_{p}\right)$ were measured from the back surface of the targets using the PDV system. Since the $U_{s}-u_{p}$ Hugoniot of aluminum 6061-T6 is available in the literature [72], it was possible to predict the shock velocities associated with Hugoniot state particle velocities measured for these impacts. The shock velocities were calculated using the empirical polynomial $U_{s}=5.35+1.34 u_{p}$ provided by Marsh [72] for aluminum 6061-T6. The $U_{s}$ and $u_{p}$ data can be seen in Table 5.2.

Three spectrograms were obtained from the plate impact experiments with aluminum 6061T6. One of these is shown in Figure 5.1 for convenience, while the other two can be seen in Appendix B. Notable features of the velocity profile seen in Figure 5.1 include: (a) the arrival of the elastic precursor wave at the free surface at $107.3 \mu \mathrm{s}$, (b) the arrival of the shock front at the free surface at $107.5 \mu \mathrm{s}$ that rapidly accelerates the free surface to the peak velocity $\left(u_{\text {peak }}\right)$ at $107.8 \mu \mathrm{s}$, (c) the velocity pullback at $108.2 \mu \mathrm{s}$, which indicates that a spall surface was formed somewhere inside the target, and (d) the oscillations in the velocity profile that follow the spall pulse. The oscillatory motion occurs as result of shock waves and rarefactions reverberating within the spall plate. The spall plate is the segment of material that exists between the free surface and the fracture surface created at the spall plane, located at some position within the target. The period of the oscillation in the velocity profile is indicative of the thickness of the spall plate. The amplitude decay is related to the damping characteristics of the interior fracture surface, where "cleaner" fracture surfaces resulting from brittle spall fracture serve to prolong the decay process [8].

An analysis of the velocity profiles obtained from experimentation was conducted to determine the dynamic mechanical properties of aluminum 6061-T6. The spall strengths measured for this material ranged between $1.34 \mathrm{GPa}$ and $1.54 \mathrm{GPa}$ for impact velocities between 580 and $667 \mathrm{~m} \cdot \mathrm{s}^{-1}$. These measured spall strengths are consistent with spall strengths ranging from 1000 to $2300 \mathrm{MPa}$ 


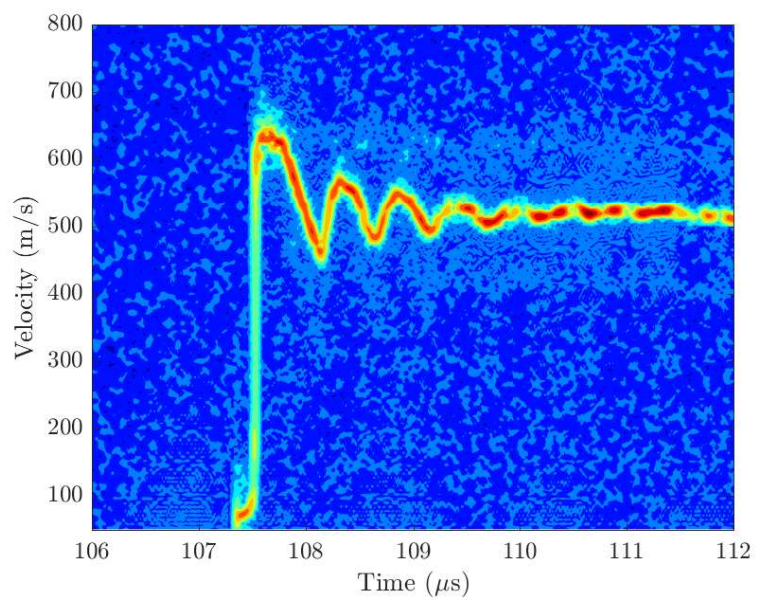

Figure 5.1: The spectrogram obtained from a plate impact experiment with an aluminum 6061T6 target impacted with an aluminum 6061-T6 flyer plate at a velocity of $633 \mathrm{~m} \cdot \mathrm{s}^{-1}$.

that are reported in the literature $[21,56]$. The large discrepancy between the maximum and minimum spall strengths reported in the literature can be attributed to the large variance of the dynamic strain rates (and shock stress) associated with the strength measurements. After all, spall strengths tend to increase with increasing strain rate and shock stress (i.e. Hugoniot state stress). The spall strengths measured for aluminum 6061-T6 are plotted against Hugoniot state stress $\left(\sigma_{H}\right)$ and tensile strain rate $(\dot{\varepsilon})$ in Figures 5.2 and 5.3, respectively.

The fracture toughness measurements of aluminum 6061-T6 ranged between 35.1 and 45.6 $\mathrm{MPa} \cdot \mathrm{m}^{1 / 2}$ for strain rates on the order of $10^{4} \mathrm{~s}^{-1}$. These values are consistent with measurements ranging from 33.0 to $48.7 \mathrm{MPa} \cdot \mathrm{m}^{1 / 2}$ reported in the literature [73, 74]. The fracture toughness measurements are plotted against Hugoniot state stress $\left(\sigma_{H}\right)$ and tensile strain rate $(\dot{\varepsilon})$ in Figures 5.4 and 5.5, respectively.

Variations in the measured spall strengths and fracture toughnesses reported here are attributed to the different suppliers that were used to source the cold-rolled aluminum plate. The aluminum plate used for shots number 1 and 3 in Table 5.2 was obtained from a separate supplier than the aluminum plate used for shot number 2 . It is possible that the plate used for shot number 2 was actually a different temper of 6061 aluminum alloy, which could explain the $\sim 190$ MPa difference in spall strength. 


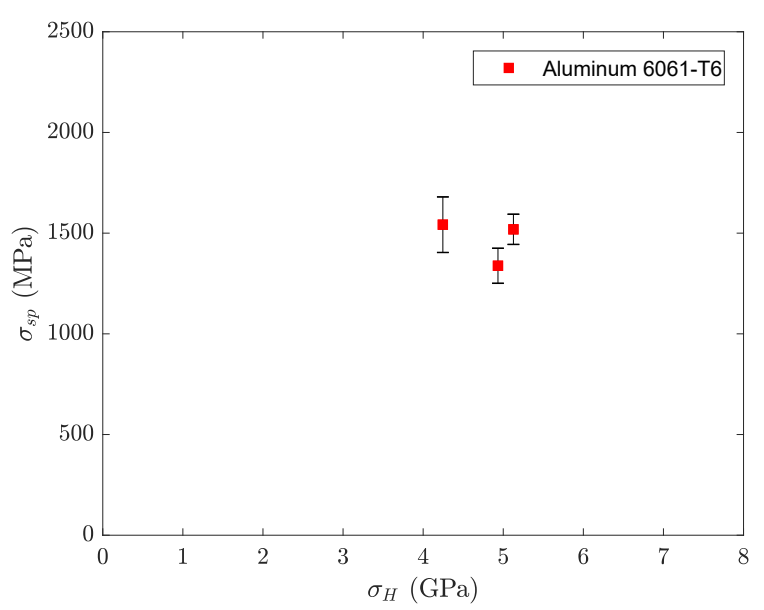

Figure 5.2: Strength measurements for aluminum 6061-T6 plotted against Hugoniot state stress.

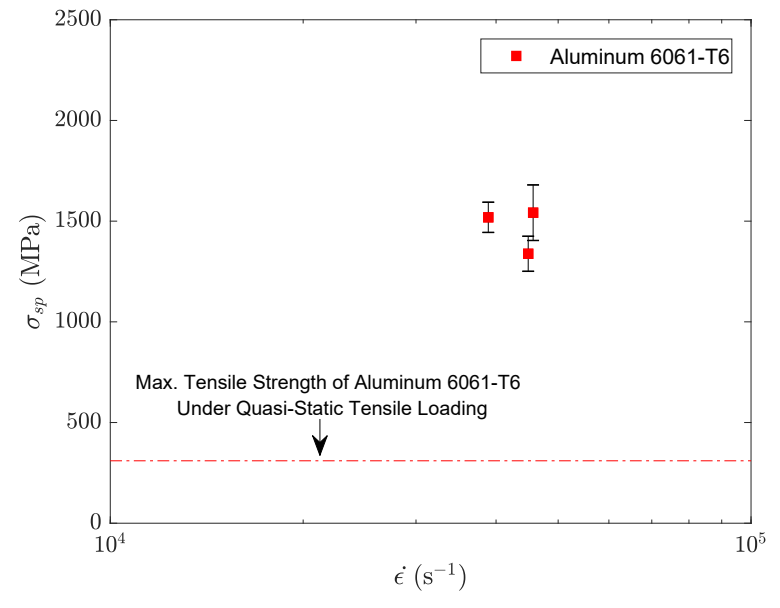

Figure 5.3: Strength measurements for aluminum 6061-T6 at low and high strain rates.

A significant difference between the quasi-static and dynamic mechanical properties of aluminum 6061-T6 can be observed in Figures 5.3 and 5.5, where values of tensile strength and fracture toughness increase dramatically between the low and high strain rate domains. Strain rate effects in aluminum 6061-T6 are well-documented [75]. Significant strengthening observed at strain rates exceeding $10^{3} \mathrm{~s}^{-1}$ is attributed to a change in the dominant dislocation motion mechanism [76]. Plastic deformation at low strain rates in crystalline materials is a energy-activated process, where microstructural obstacles (such as grain boundaries and secondary phases), as well as the Peierls stress barrier control dislocation mobility [76]. Increases in local energy can allow pinned dislocations to escape from one barrier and glide to the next. Conversely, plastic deformation at high strain rates is controlled by viscous phonon drag $[76,77]$. Phonon drag describes an increase in the relative mass of electrons caused by an increased interaction with the crystal lattice through which the electrons move [78]. As electrons translate past atoms hosted in the lattice, their charge polarizes the adjacent lattice [78]. This causes a decrease in electron mobility and a subsequent reduction in dislocation mobility, which serves to increase the material's inherent resistance to plastic deformation. As this occurs, the formation of cracks or voids within the bulk of the target is prolonged, raising the maximum tensile stress that is achieved from the interaction of the rarefaction fans. Therefore the increase in tensile strength that is observed when jumping 


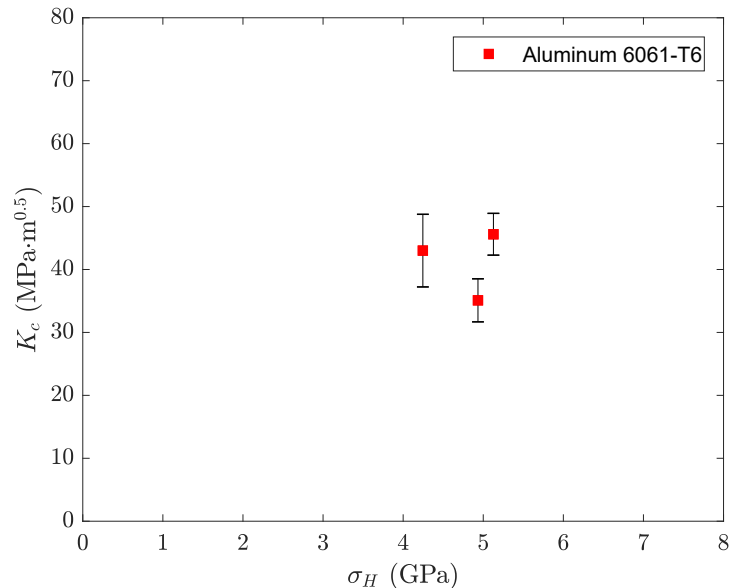

Figure 5.4: Fracture toughness measurements for aluminum 6061-T6 plotted against Hugoniot state stress.

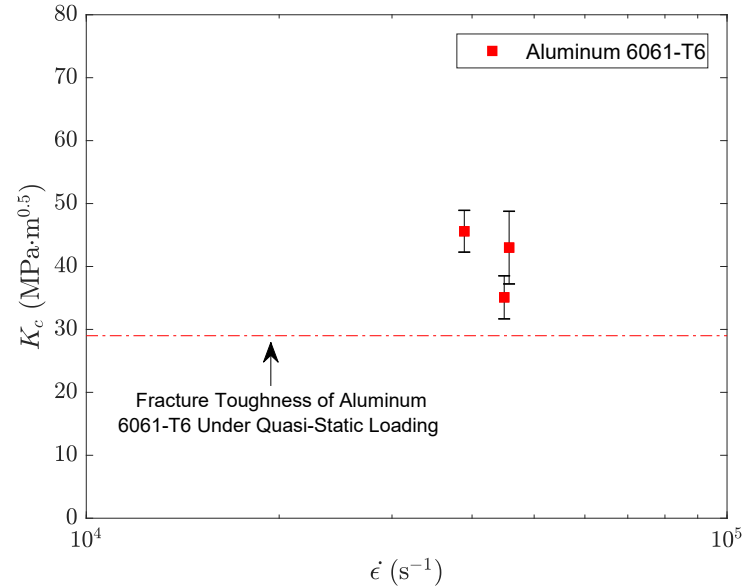

Figure 5.5: Fracture toughness measurements for aluminum 6061-T6 at low and high strain rates.

from low to high strain rates can be attributed to reduction in dislocation mobility caused by the rate-induced change in the dominant dislocation motion mechanism.

The successful identification of the spall strengths and fracture toughnesses for aluminum 6061-T6 validated the experimental and analytical methods used in this study. With these methods established, the focus of the research shifted towards investigating the shock response and dynamic failure behaviour of polymethylmethacrylate (PMMA). The results of the plate impact experiments conducted with PMMA are presented in the following section.

\subsection{Polymethylmethacrylate}

The experimental setup used for several of the experiments with PMMA targets did not included shock pins. This meant that, for those shots, the time of impact (TOI) of the flyer plate was not identified, making it impossible to accurately determine shock transit times in the specimens from experimentation. Fortunately, the Hugoniot state particle velocities $\left(u_{p}\right)$ were measured from the back surface of the targets using the PDV system. Since the $U_{s}-u_{p}$ Hugoniot of PMMA is available in the literature [32], it was possible to predict the shock velocities associated with the Hugoniot 


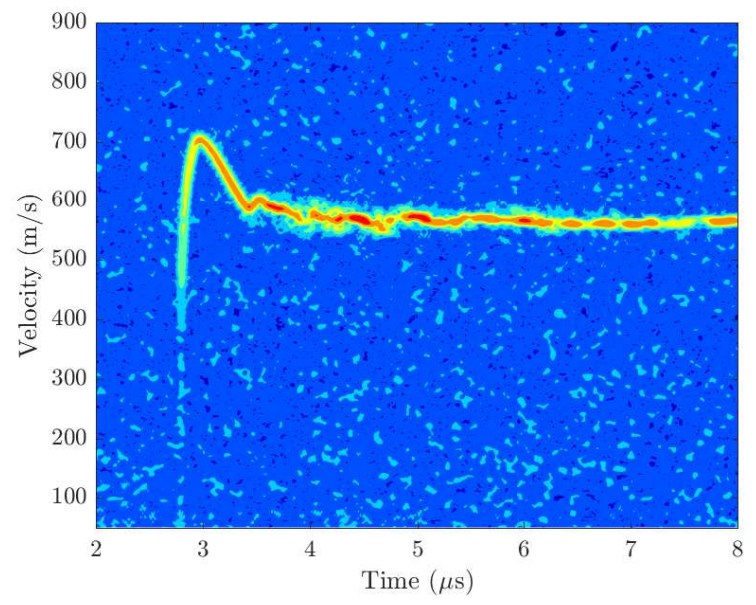

Figure 5.6: The spectrogram of a PMMA target impacted with an aluminum 6061-T6 flyer plate at a velocity of $502 \mathrm{~m} \cdot \mathrm{s}^{-1}$.

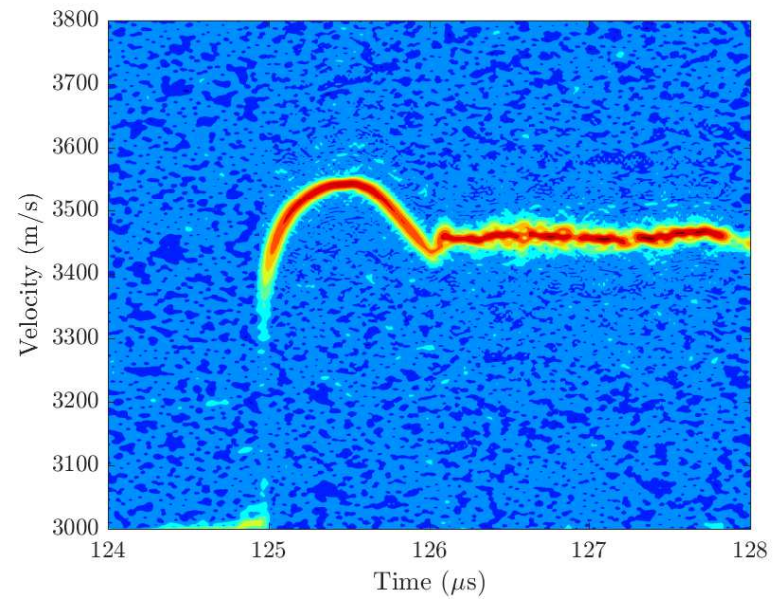

Figure 5.7: The spectrogram of a PMMA target impacted with a PMMA flyer plate at a velocity of $563 \mathrm{~m} \cdot \mathrm{s}^{-1}$.

state particle velocities measured for these impacts. The shock velocities for shots performed without shock pins were calculated using the empirical polynomial for PMMA, $U_{s}=6.486 u_{p}^{3}-$ $7.823 u_{p}^{2}+3.549 u_{p}+2.703$ for $u_{p}<0.4 \mathrm{~km} \cdot \mathrm{s}^{-1}$ provided by Jordan et al. [72]. The measured and theoretically-predicted $U_{s}$ and $u_{p}$ data for PMMA can be seen in Table 5.2.

While the complete collection of spectrograms obtained for shots with PMMA can be viewed in Appendix B, two images from that collection with features of particular interest are shown here in Figures 5.6 and 5.7. The velocity profile seen in Figure 5.6 shows spall behaviour that is typical for amorphous polymers, where the slight velocity recovery after the velocity pullback at $3.4 \mu \mathrm{s}$ is followed by minimal velocity oscillation from the spall plate effect. The velocity profile seen in Figure 5.7 displays similar behaviour, with its distinguishing feature being the increased plastic shock wave rise time. Here, rise time was defined as the interval measured between the time of the end of the elastic precursor wave and the time at which $75 \%$ of the peak velocity was achieved on the free surface velocity profile. The rise time for this impact represented by Figure 5.7 is approximately $50 \pm 10 \mathrm{~ns}$, whereas the rise time for the impact represented by Figure 5.6 is $25 \pm 10$ ns. This is attributed to the use of a PMMA flyer plate, which generated a symmetrical impact $\left(Z_{\text {flyer }}=Z_{\text {target }}\right)$. This phenomenon is also observed for the plate impact experiments conducted with epoxy targets discussed in the next section. 


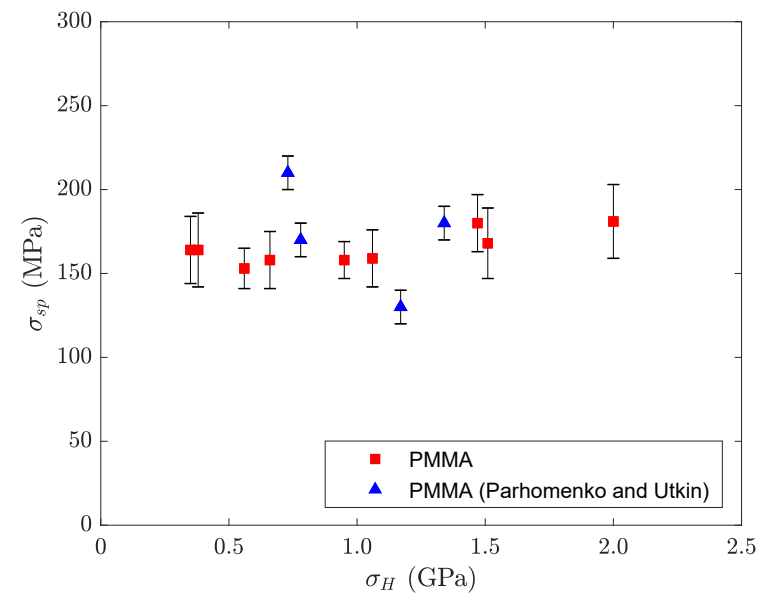

Figure 5.8: Strength measurements for PMMA plotted against Hugoniot state stress.

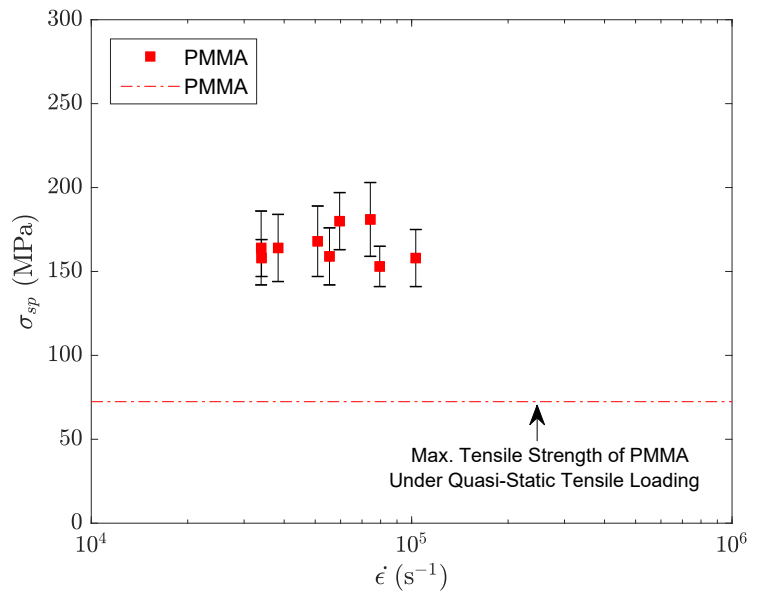

Figure 5.9: Strength measurements for PMMA at low and high strain rates.

Parhomenko and Utkin [43] conducted several spall experiments with PMMA specimens using plate impact and explosive shock-loading techniques. The free surface velocity profiles obtained from their experiments possess features that are effectively identical to those seen on the velocity profiles in Figures 5.6 and 5.7. An analysis of the velocity profiles retrieved from experimentation was conducted to determine the dynamic tensile properties of PMMA. The spall strengths measured for this material ranged between 153 and $181 \mathrm{MPa}$ for impact velocities between 195 and $722 \mathrm{~m} \cdot \mathrm{s}^{-1}$. It should be noted that the spall strength measured for shot number 7 in Table 5.2 was rejected due to the lack of a clear pullback velocity on the velocity profile. The spectrogram for this shot can be seen in Figure 5.10. These values are consistent with spall strengths of 130 to 210 MPa reported by Parhomenko and Utkin [43]. The spall strengths for PMMA are plotted against Hugoniot state stress in Figure 5.8 and against strain rate in Figure 5.9.

The fracture toughness of PMMA was found to range between 2.21 and $3.75 \mathrm{MPa} \cdot \mathrm{m}^{1 / 2}$ for strain rates on the order of $10^{4} \mathrm{~s}^{-1}$. These values are consistent with fracture toughnesses of 2.5 to 4.1 $\mathrm{MPa} \cdot \mathrm{m}^{1 / 2}$ published by Weerasooriya et al. [62] for PMMA. The fracture toughnesses are plotted against Hugoniot state stress in Figure 5.11 and against strain rate in Figure 5.12.

Large differences between the dynamic and quasi-static mechanical properties of PMMA can be observed in Figures 5.9 and 5.12. A thorough explanation for this behaviour is provided in 


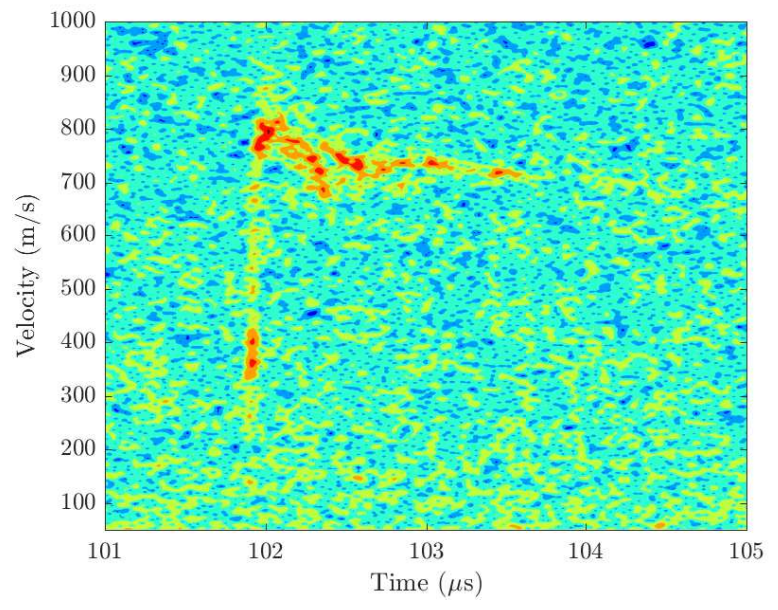

Figure 5.10: A PMMA target impacted with an aluminum 6061-T6 flyer plate at a velocity of $656 \mathrm{~m} \cdot \mathrm{s}^{-1}$.

section 5.3.1, which attributes the observed phenomenon to a strengthening effect caused by a strain rate-induced restriction of molecular mobility.

The present measurements of spall strength and fracture toughness in PMMA are in agreement (within experimental uncertainty) with the values reported in the literature [43, 62]. From here, the focus of the experimental investigation shifted towards studying the shock response and dynamic failure behaviour of materials whose properties were previously unknown. These materials included a bisphenol-A epoxy resin cured with two different curing agents, as well as an epoxy/carbon nanotube composite. The results of these experiments are presented in the following sections.

\subsection{EPON 828 Epoxy}

The shock transit times measured in the epoxy targets from the plate impact experiments were used to determine the shock Hugoniots of the test materials. The shock Hugoniot data for the two EPON 828 epoxy systems are plotted against data obtained by Carter and Marsh [33] (curing agent not specified) in Figure 5.13. These results demonstrate that the shock Hugoniot is not particularly sensitive to the curing agent in this epoxy. The particle velocity was determined via two methods 


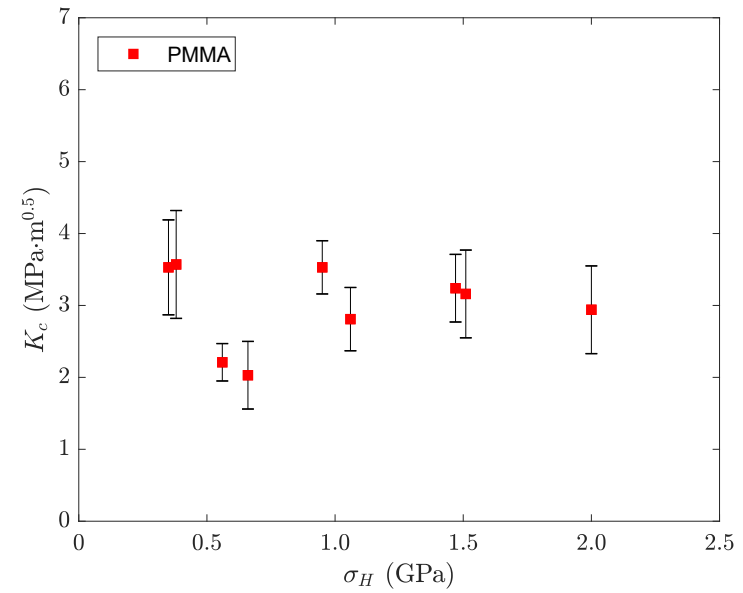

Figure 5.11: Fracture toughness measurements for PMMA plotted against Hugoniot state stress.

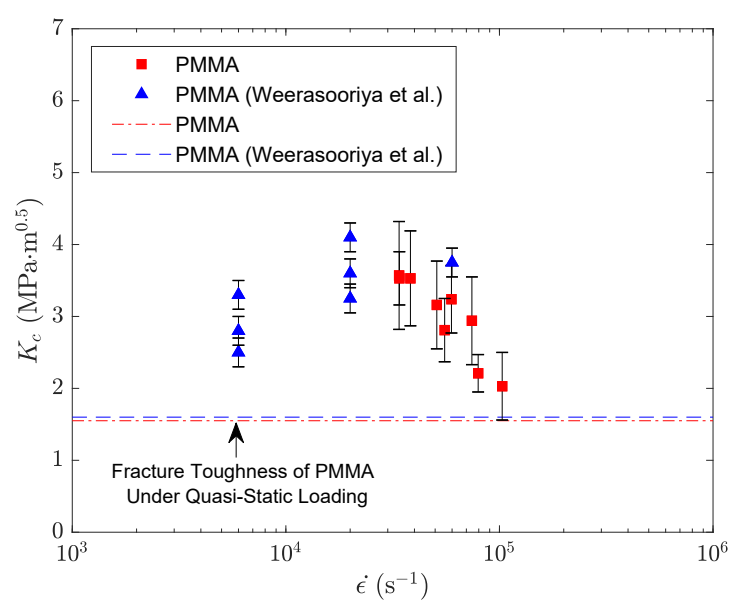

Figure 5.12: Fracture toughness measurements for PMMA at low and high strain rates.

for verification. Direct measurement from the back face PDV was compared to values calculated from an analytical impedance-matching technique [52] using the approach described by Petel and Higgins [79]. This approach implements the idea that $u_{\text {peak }}$ measured for a symmetrical (i.e., impedance-matched) impact should be equal to the impact velocity $\left(v_{i}\right)$ of the flyer plate. The resulting particle velocity would then equal half of the peak velocity, according to the intersection of the left-going $P-u_{p}$ Hugoniot in the flyer plate and right-going $P-u_{p}$ Hugoniot in the target. Overall, these two approaches were in close agreement.

The shock Hugoniot measurements from the present study (Figure 5.13) are in agreement, within experimental uncertainty, with data reported previously for EPON 828 [33]. This implies that the choice of curing agent had little or no effect on the shock response of the epoxy system. This is consistent with the work of Munson and May [41], where it was shown that variations in the molecular structure of the polymer chains (caused by changing the curing agent) had no effect on the high-pressure compression response of the resulting epoxy systems. They deduced from their results that the curing agents had no appreciable effect on the equilibrium positions of the backbone chains. Similar reasoning can be used to explain the results of the present study. One can use the $U_{s}-u_{p}$ relationship to comparatively discuss material compressibility, so the results can be interpreted as confirming a lack of variation in the equilibrium spacing of adjacent backbone 


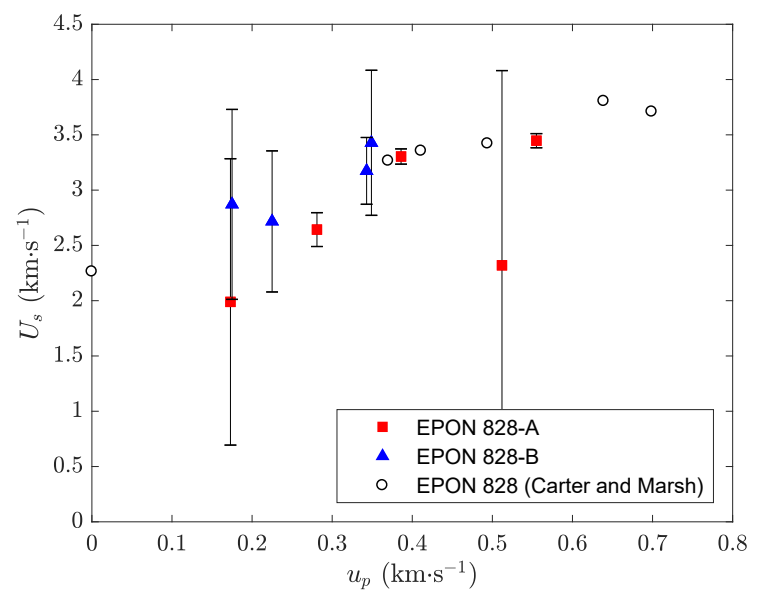

Figure 5.13: $U_{s}$ and $u_{p}$ measurements for both EPON 828 variants plotted with data by Carter and Marsh for comparison [33].

chains between EPON 828-A and EPON 828-B.

Four representative spectrograms are given in Figures 5.14, 5.15, 5.16, and 5.17. Asside from the dominant signal, the spectrograms feature high-velocity horizontal signal bands at the free surface following shock arrival. These bands are reminiscent of PDV signals for ejecta plumes and are interpreted as ejecta from the sputtered aluminum coating on the target [80]. Reducing the surface roughness by wet polishing the EPON 828 specimens with sandpaper prior to sputtering was found to lessen the severity of the ejecta plume.

There are two main types of results observed in the spectrogram images: sub-critical (i.e., non-spall) impacts and spall events. Figure 5.14 is considered a non-spall event, identified by the oscillatory velocity profile and the absence of a characteristic spall (i.e., velocity pullback) signal on the free surface velocity profile. Figures 5.15, 5.16, and 5.17, however, are all spectrograms of spall events that exhibit the same features of the idealized signal shown in Figure 3.12 (see Chapter 3).

An analysis of all the velocity profiles retrieved from experimentation with EPON 828-A and EPON 828-B was conducted to determine their dynamic mechanical properties. The spall strengths for EPON 828-A and EPON 828-B were found to range from 404 to $585 \mathrm{MPa}$ and from 339 to $397 \mathrm{MPa}$, respectively. The fracture toughness of EPON 828-A ranged between 14.8 and 


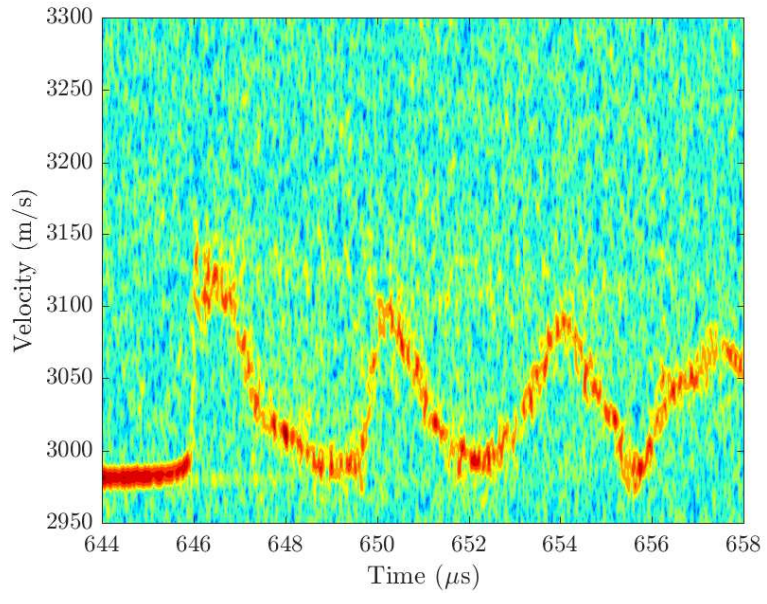

Figure 5.14: An EPON 828-A target impacted with a PMMA flyer plate at a velocity of $136 \mathrm{~m} \cdot \mathrm{s}^{-1}$. A baseline velocity offset of $2984 \mathrm{~m} \cdot \mathrm{s}^{-1}$ was applied.

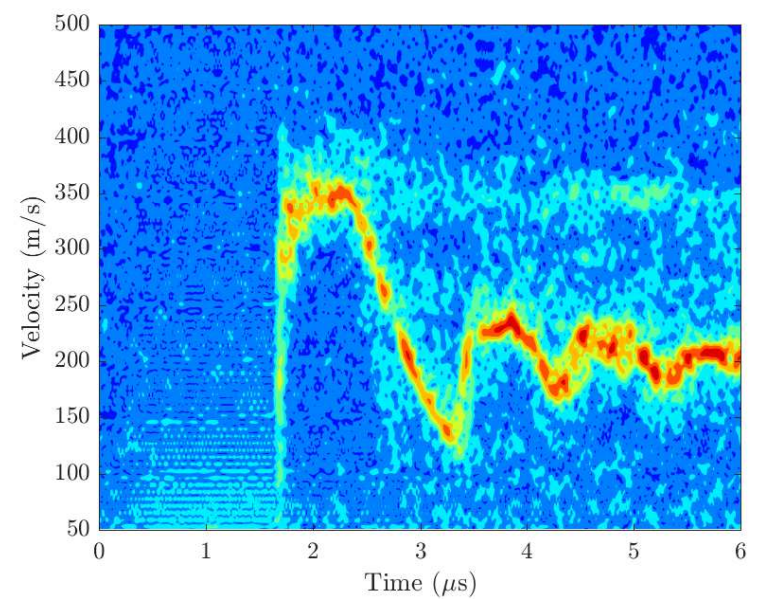

Figure 5.16: An EPON 828-B target impacted with a PMMA flyer plate at a velocity of $344 \mathrm{~m} \cdot \mathrm{s}^{-1}$.

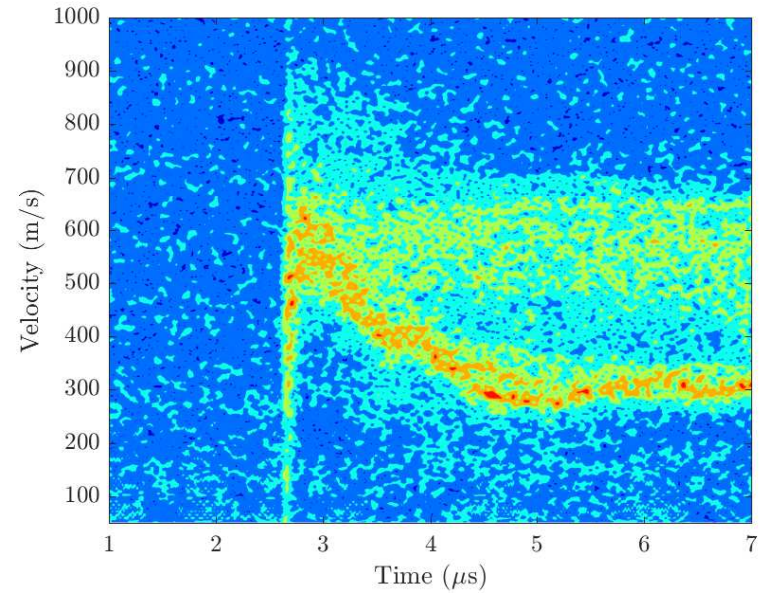

Figure 5.15: An EPON 828-A target impacted with an aluminum 6061-T6 flyer plate at a velocity of $363 \mathrm{~m} \cdot \mathrm{s}^{-1}$.

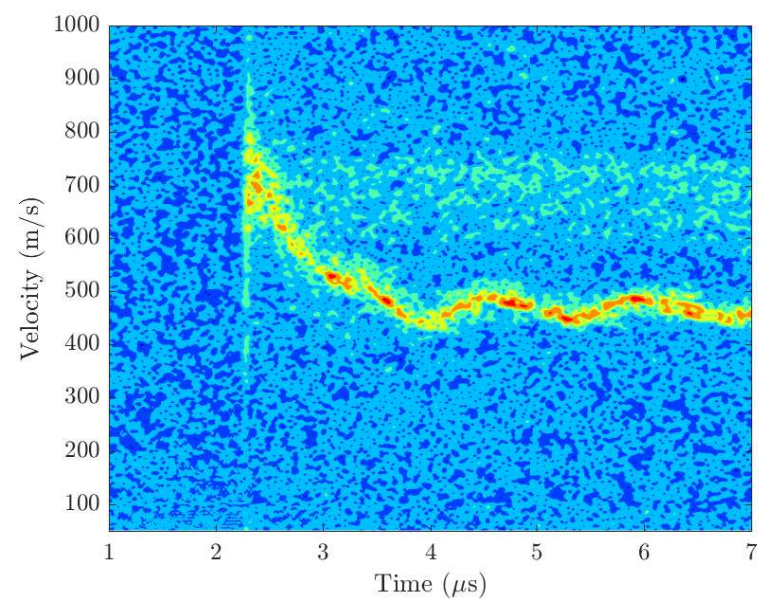

Figure 5.17: An EPON 828-B target impacted with an aluminum 6061-T6 flyer plate at a velocity of $471 \mathrm{~m} \cdot \mathrm{s}^{-1}$.

19.0 MPa $\cdot \mathrm{m}^{1 / 2}$ for strain rates on the order of $10^{4} \mathrm{~s}^{-1}$, whereas the values for EPON 828-B were slightly lower, ranging between 8.95 and $11.6 \mathrm{MPa} \cdot \mathrm{m}^{1 / 2}$. The complete set of the experimental results for EPON 828-A and B can be found in Table 5.2.

From Figure 5.18, the spall strengths measured in both EPON 828-A and EPON 828-B were found to be considerably higher than those of PMMA. These results also demonstrate that the spall strengths of the epoxies are strongly dependent on the curing agent. In Figure 5.19, the results 


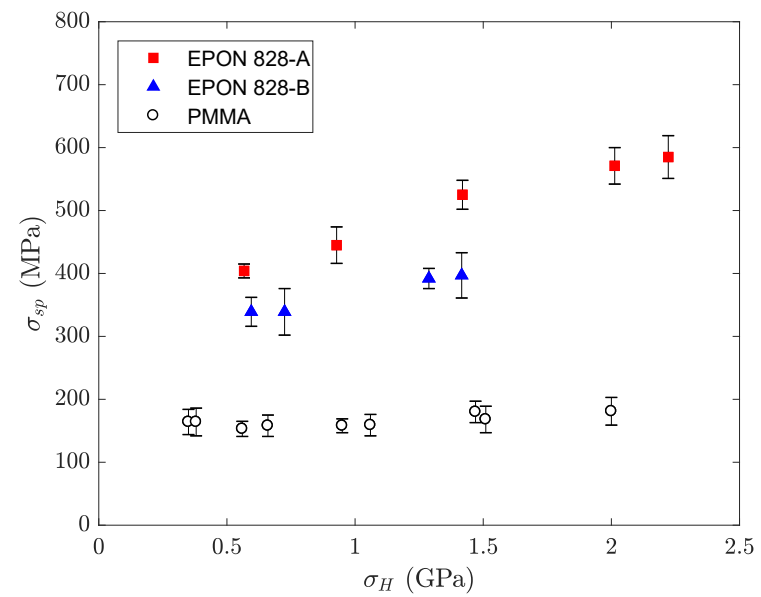

Figure 5.18: Strength measurements for EPON 828-A, EPON 828-B, and PMMA plotted against Hugoniot state stress.

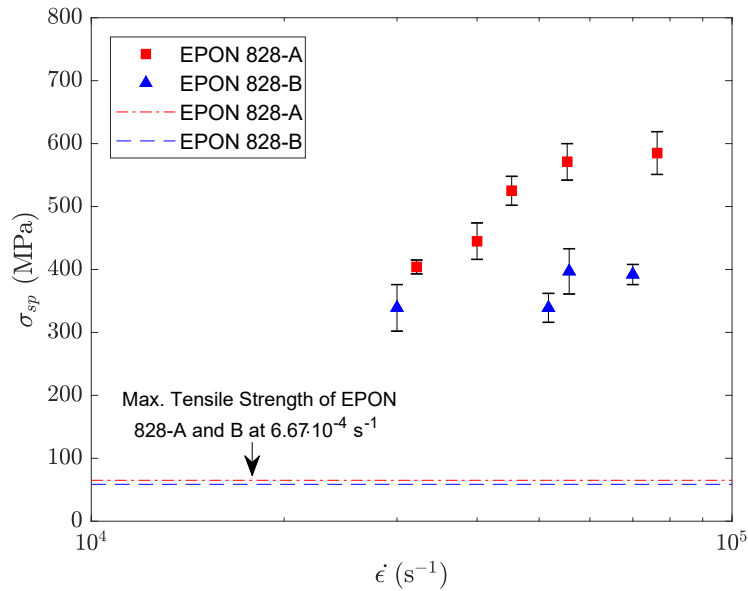

Figure 5.19: Strength measurements for EPON 828-A and EPON 828-B at low and high strain rates.

demonstrate a strain-rate dependence of the spall strengths for both epoxies, which again differ from the response of PMMA.

The spall strength of EPON 828 has been previously reported to be $76 \mathrm{MPa}$ [44, 81], which is significantly lower than the average value of $444 \mathrm{MPa}$ reported here. There are a few possible explanations for this discrepancy between the results of the present study and those reported by Guess [44]. The strong influence of the curing agent on the spall strength is evident from Figures 5.18 and 5.19, where the spall strength of EPON 828-A was, on average, 29\% higher than that of EPON 828-B. Such a considerable difference between contemporary curing agents for a resin system (which have been optimized throughout years of research) may explain the even larger variation between studies resulting from the choice of curing agent for the epoxy.

An alternative explanation is the influence of the flyer plate material on the interpretation of the spall signal. The choice of flyer plate material will influence the free surface velocity profile, which can lead to the misidentification of the pullback velocity $\left(u_{\text {pull }}\right)$. The common approach to spall testing involves impacting the specimen with a thin metallic flyer plate. When the test material is a metal, the backface velocity signal is often identical to the idealized velocity profile shown in Figure 3.12 in Chapter 3, with a sharp increase in the velocity to signal the spall event. The backface velocity profiles of spall tests with polymers often feature only a slight velocity increase 


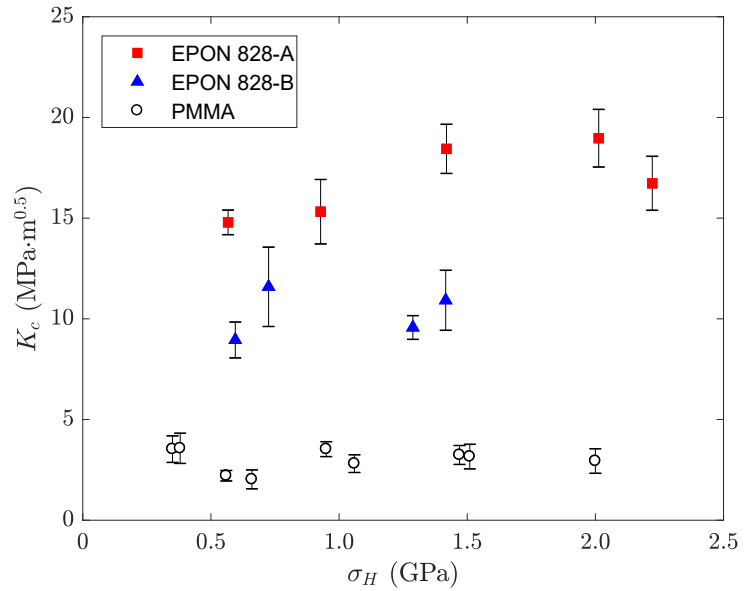

Figure 5.20: Fracture toughness measurements for EPON 828-A, EPON 828-B, and PMMA plotted against Hugoniot state stress.

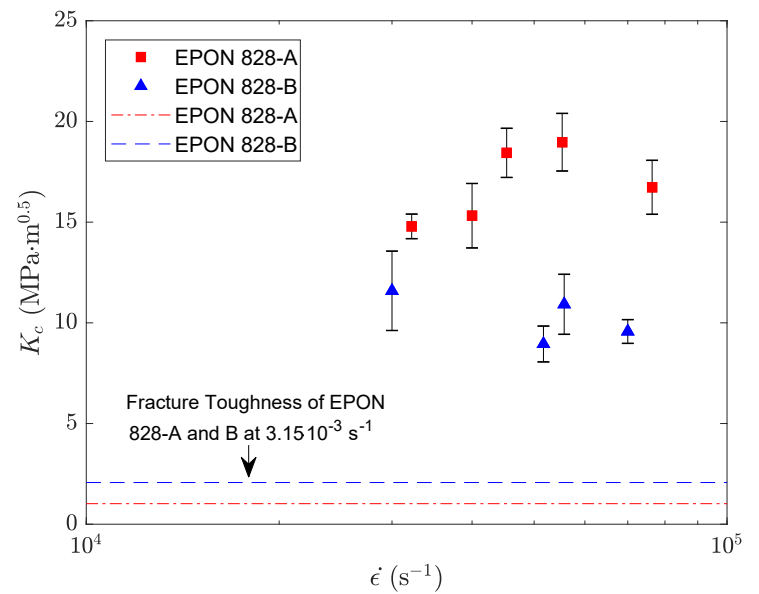

Figure 5.21: Fracture toughness measurements for EPON 828-A and EPON 828-B at low and high strain rates.

following a spall event, which is seen in Figure 5.15.

Another feature of note that is seen in both Figures 5.15 and 5.17 are the velocity plateaus in the release signal from the test material. In Figure 5.15, this feature is seen at $3.5 \mu \mathrm{s}$ and a velocity near $400 \mathrm{~m} \cdot \mathrm{s}^{-1}$, while it is seen at $3 \mu \mathrm{s}$ and $520 \mathrm{~m} \cdot \mathrm{s}^{-1}$ in Figure 5.17 . These features are only seen for an aluminum flyer and are not present in the release velocity profile in tests with the PMMA flyer plate (Figure 5.16), resulting in a less ambiguous spall signal. An analysis of the wave dynamics within the test system will show that the plateaus seen for the aluminum flyer tests are the result of the finite thickness of a flyer plate with an impedance that is significantly higher than the test material and are not indicative of the spall event. The first minimum in backface velocity, rather than a plateau, was taken for the spall strength estimate. Using two different flyer plates for the same impact velocity range (see shot 13 and shot 16 in Table 5.2), shows that this approach results in consistent measurements. Choosing the plateau velocity from tests with aluminum flyer plates resulted in spall strengths that were much closer, but still greater than the strength published by Guess [44], however the results were inconsistent with PMMA flyer plate tests. The effect of the flyer plate material on the interpretation of spall signals is a possible cause of the discrepancies between the values reported in the present study and those published previously [44]. 


\subsubsection{Strain Rate Effects}

A significant difference between the quasi-static and dynamic mechanical properties of EPON 828A and EPON 828-B can be observed in Figures 5.19 and 5.21, where values of tensile strength and fracture toughness increase by approximately one order of magnitude between the low and high strain rate domains. The same response is seen for PMMA, albeit to a lesser extent. Interestingly, while the quasi-static tensile strength of PMMA is slightly higher than that of either EPON 828 variant (Table 4.1), the opposite is seen among the spall strengths of the materials, where the EPON 828 variants have higher dynamic tensile strengths. A pronounced strain rate dependency is also observed in the fracture toughness measurements, where the epoxies are significantly tougher than PMMA at high strain rates, but perform similarly to it under quasi-static loading conditions.

Pronounced microstructure-dependent strain rate effects are well-documented for thermoplastic and thermosetting polymers [5, 62, 82, 83]. The Ree-Eyring theory [84] is quite applicable here, which is an analytical model that describes the flow of a material as being controlled by the simultaneous interactions of multiple rate-activated processes. When applying this theory to plasticity in polymers, these processes are assumed to be related to specific degrees of freedom of the backbone chains. From this, the transition in yield behaviour observed from quasi-static to dynamic loading conditions is explained in terms of molecular mobility. Resistance to material deformation in the context of a high strain rate deformation of a polymer has been attributed to the restriction of the degrees of freedom within a polymer chain [62]. Thus, the strengthening and toughening effects observed in EPON 828-A, EPON 828-B, and PMMA can be attributed to the restriction of the mobility of the individual molecular chains at high strain rate. A similar effect is seen in dense particle suspensions under high-strain-rate loading, where the sudden jamming of their structure results in a significant increase in stiffness [79, 85].

\subsubsection{Effect of Curing Agent}

The results of the present study clearly demonstrates that the choice of curing agent has a direct effect on the quasi-static and dynamic mechanical properties of the resultant epoxy, despite its 
negligible influence on shock wave propagation. Among the EPON 828 variants, EPON 828-A has consistently higher tensile strength than EPON 828-B at any given strain rate. In terms of fracture toughness, the results are more complex. While EPON 828-B has a higher fracture toughness than EPON 828-A under quasi-static loading conditions, the opposite trend is seen for fracture toughness, with EPON 828-A exhibiting a larger fracture toughness under dynamic loading.

The distinguishing features of these two curing agents may provide an explanation of this

behaviour. Millett et al. [36] demonstrated that variation in the atoms attached to the polymer backbone in thermoplastics can change their macroscopic dynamic behaviour. Functionalizing the backbone chains via the introduction of a curing agent to encourage crosslinking, reduction of free volume, or reduction of the characteristic spacing could potentially result in variation in the binding forces experienced between adjacent backbone chains. Variation in the binding forces would likely translate to differences in macroscopic mechanical properties, including spall strength and fracture toughness. This provides a possible explanation for the observed behaviour, where one curing agent may promote more or less crosslinking between backbone chains than another, which would result in variation in the dynamic mechanical properties between the different epoxy systems. Following this explanation leads to the likely conclusion that EPON 828-A has greater crosslinking between adjacent polymer chains than EPON 828-B.

\subsection{Epoxy/Carbon Nanotube Composite}

The shock transit times measured from the plate impact experiments were used to determine the shock velocities within the nanocomposite targets, and subsequently establish the $U_{s}-u_{p}$ Hugoniot. The $U_{s}$ and $u_{p}$ measurements shown in Figure 5.22 are plotted alongside measurements for neat EPON 828-A epoxy, and with measurements published by Carter and Marsh [33].

As seen in Table 5.2, the measured particle velocities $\left(u_{p}\right)$ were equivalent to the impact velocities (within experimental error) for shots conducted with PMMA flyer plates. This was expected due to the similar shock impedances $(Z)$ of PMMA and EPON 828-A. The shock velocities $\left(U_{s}\right)$, 


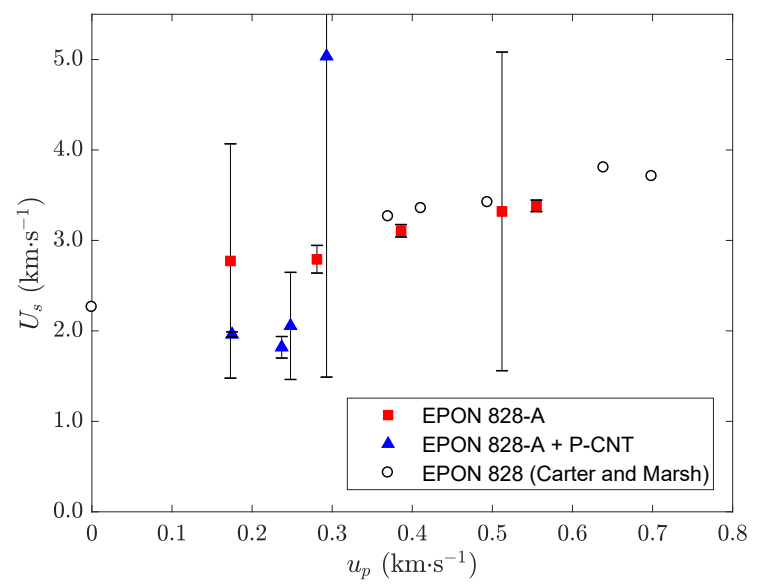

Figure 5.22: $U_{s}$ and $u_{p}$ measurements for EPON 828-A and the EPON 828-A + P-CNT composite plotted with data by Carter and Marsh for comparison [33].

however, show significant scatter. This is attributed to the relatively thin targets that were used. The ideal flyer plate-to-target thickness ratio $\left(w_{f}: w_{t}\right)$ for a symmetrical impact is approximately $1: 5$ [8]. This is established to help ensure the formation of a triangular shock front, which is an assumption inherant to Stepanov's model for spall strength (see Eq. 3.18) [59]. The average $w_{f}: w_{t}$ ratio for the impacts conducted with the epoxy/carbon nanotube composite was $1: 2.6$. The undersized targets also enhanced the error associated with measuring of the shock transit times, an error that compounded further when computing the shock velocities. Therefore, due to significant error in the measurement of shock transit times, a $U_{s}-u_{p}$ Hugoniot was not provided for the epoxy/carbon nanotube composite, since it would not accurately represent the shock-response of the material.

A total of six spectrograms were obtained from the plate impact experiments conducted with nanocomposite targets. While the complete collection of these spectrograms can be seen in Appendix B, two representative spectrograms are shown here in Figures 5.23 and 5.24. Unfortunately, only four of the six total spectrograms were used to examine the failure behaviour of the material. This is because issues with the experimental setup used for shots number 28 and 30 (see Table 5.2) were noticed only after examining their velocity profiles, which are shown in Figures 5.25 and 5.26, respectively. For shot number 28 (Figure 5.25), the free surface of the target was coated with a white enamel spray paint to make the material visible to the PDV system. Dominant beat 


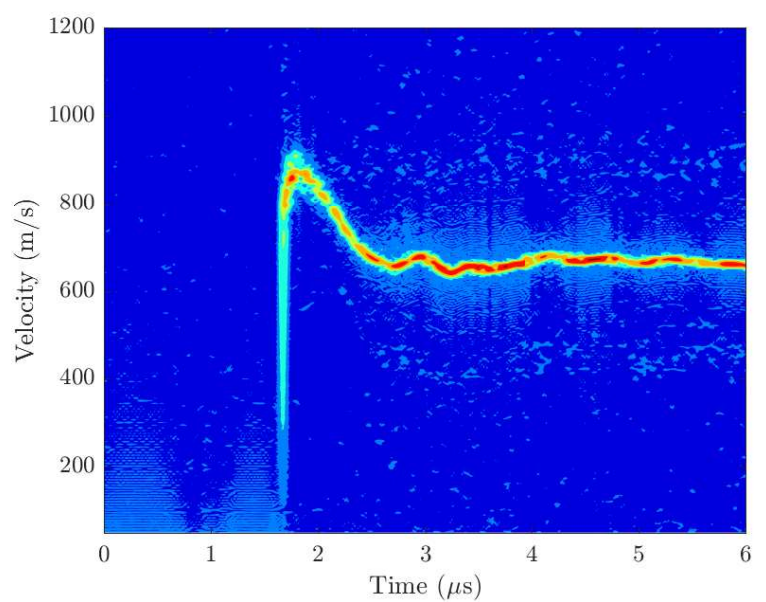

Figure 5.23: An EPON 828-A + P-CNT composite target impacted with an aluminum 6061-T6 flyer plate at a velocity of 617 $\mathrm{m} \cdot \mathrm{s}^{-1}$.

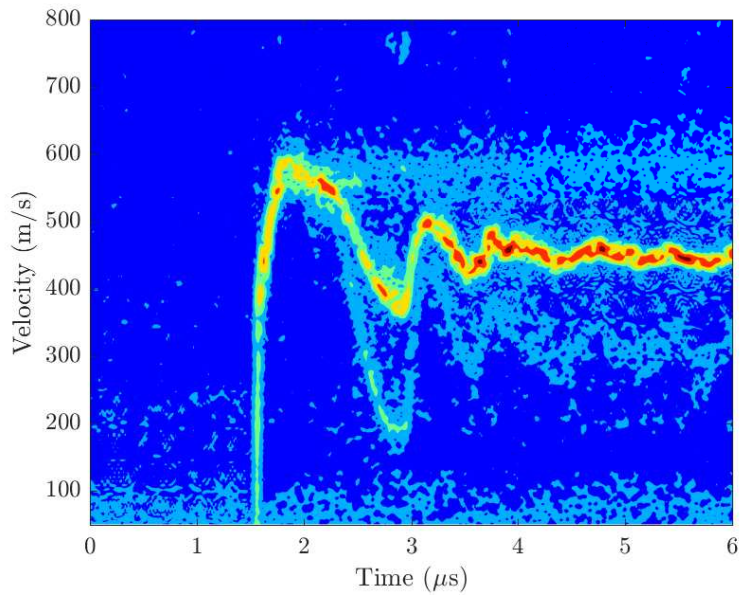

Figure 5.24: An EPON 828-A + P-CNT composite target impacted with a PMMA flyer plate at a velocity of $625 \mathrm{~m} \cdot \mathrm{s}^{-1}$.

frequencies associated with the motion of paint ejected from the free surface (upon arrival of the initial shock front) are visible on the spectrogram for this shot. This indicates that the paint formed a relatively weak bond with the composite surface. The lack of a dominant well-resolved velocity profile made it impossible to confidently identify the peak and pullback velocities. The spall strength computed for this shot using these velocities therefore had significant error. Hence, this spall strength was not considered when examining the failure behaviour of the nanocomposite.

The spectrogram associated with shot number 30 (Figure 5.26) was also deemed unacceptable due to the relatively low magnitude of the $\Delta u_{f s}$ parameter obtained from analysis of the velocity profile. For this particular shot, a sheet of aluminized Mylar® foil was adhered to the free surface of the nanocomposite target using a five-minute epoxy (Loctite ${ }^{\circledR}$ EA E-05CL), rather than superglue. It is suspected that the five-minute epoxy formed a weak bond between the Mylar® foil and the composite, resulting in the premature ejection of the foil from the free surface. Therefore, the pullback velocity observed on the spectrogram from this shot is believed to represent spall fracture of the five-minute epoxy, rather than spall fracture of the nanocomposite. Therefore the analysis of this velocity profile would not provide useful information. This hypothesis is supported by the rapid period of the oscillations in the velocity history observed between 2.7 and $3.3 \mu$ s, which 


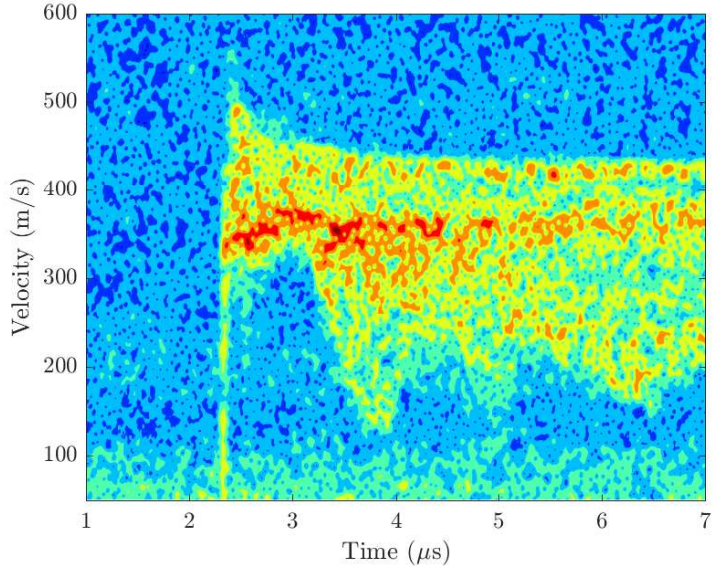

Figure 5.25: An EPON 828 / EPIKURE 3223 epoxy + 1 wt. \% P-CNT composite target impacted with a PMMA flyer plate at a velocity of $346 \mathrm{~m} \cdot \mathrm{s}^{-1}$.

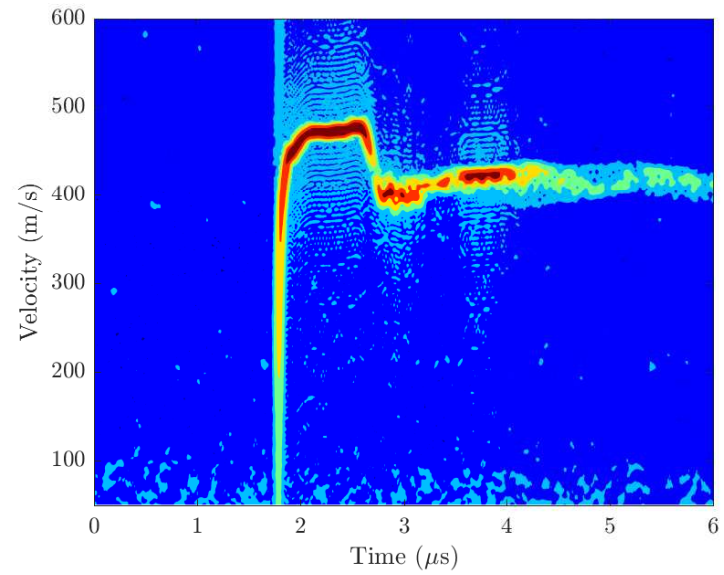

Figure 5.26: An EPON 828 / EPIKURE 3223 epoxy +1 wt. \% P-CNT composite target impacted with a PMMA flyer plate at a velocity of $483 \mathrm{~m} \cdot \mathrm{s}^{-1}$.

indicates that the PDV registered shock wave and rarefaction reflections within an unusually thin spall plate, or more likely: the sheet of ejected aluminized Mylar® foil. For this reason, all measurements obtained from this shot (spall strength, fracture toughness, etc.) were not considered when studying the dynamic tensile failure behaviour of the nanocomposite.

An analysis of the velocity profiles retrieved from experimentation was conducted to determine the dynamic tensile properties of the epoxy/carbon nanotube composite. The spall strengths measured for this material ranged between 291 and $392 \mathrm{MPa}$ for impact velocities between 353 and $625 \mathrm{~m} \cdot \mathrm{s}^{-1}$. These spall strengths are plotted against Hugoniot state stress $\left(\sigma_{H}\right)$ and tensile strain rate $(\dot{\varepsilon})$ in Figures 5.27 and 5.28, respectively. Spall strength measurements published by Bie et al. [5] for a similar bisphenol-A epoxy (neat) and a nanocomposite comprising of bisphenol-A epoxy mixed with 1 wt.\% pristine multi-walled carbon nanotubes (P-CNT) are also plotted in Figure 5.27. Those strengths range from 206 to $222 \mathrm{MPa}$ for impacts conducted with epoxy flyer plates at velocities between 171 and $220 \mathrm{~m} \cdot \mathrm{s}^{-1}$, respectively. The carbon nanotubes used in their study were $1 \mu \mathrm{m}$ to $10 \mu \mathrm{m}$ in length, and had outer and inner diameters of $15 \mathrm{~nm}$ and $4 \mathrm{~nm}$, respectively (making them larger than the nanotubes used in the present study).

Bie et al. [5] also tested epoxy nanocomposite fabricated with nanotubes that had been func- 


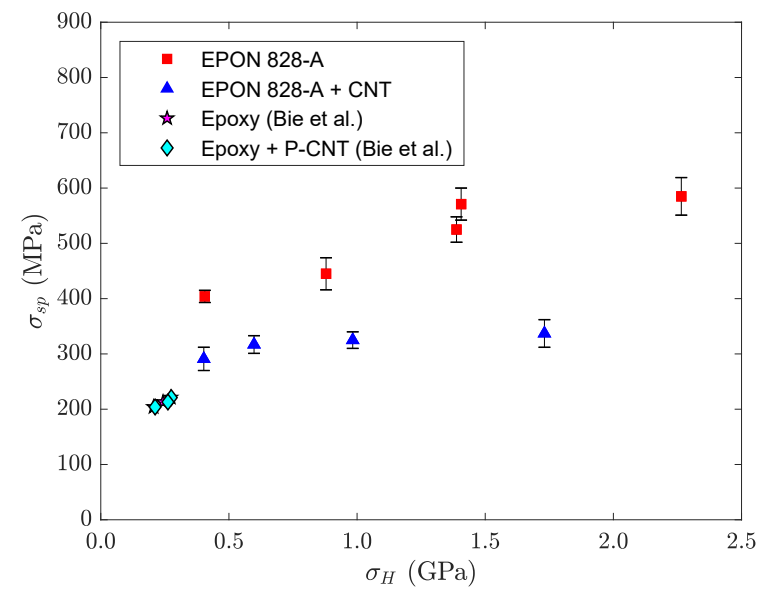

Figure 5.27: Strength measurements for neat EPON 828-A and EPON 828-A + P-CNT composite plotted against Hugoniot state stress.

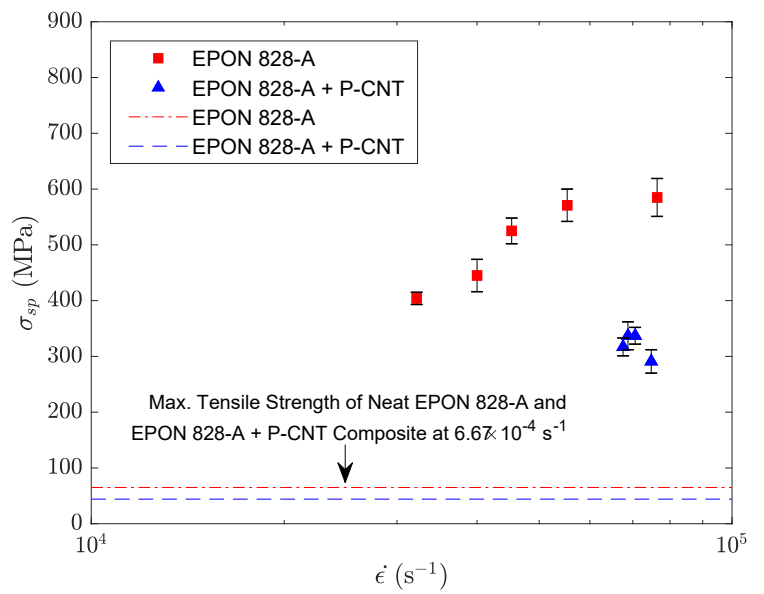

Figure 5.28: Strength measurements for neat EPON 828-A and EPON 828-A + P-CNT composite at low and high strain rates.

tionalized with ozone (henceforth referred to as F-CNT). The intention of this treatment was to improve the bond strength of the epoxy/carbon nanotube interface. The spall strengths measured for the F-CNT nanocomposite ranged from 174 to $210 \mathrm{MPa}$, which indicates that functionalizing the nanotubes with ozone had a slight detrimental effect on the spall strength of the nanocomposite.

The fracture toughness measurements of EPON 828-A + P-CNT ranged between 5.95 and 7.75 $\mathrm{MPa} \cdot \mathrm{m}^{1 / 2}$. These measurements are plotted against Hugoniot state stress $\left(\sigma_{H}\right)$ and tensile strain rate $(\dot{\varepsilon})$ in Figures 5.29 and 5.30, respectively. Fracture toughness measurements published by Bie et al. for the P-CNT nanocomposite described earlier are also plotted in Figure 5.29 [5]. Measurements for the P-CNT nanocomposite ranged from 2.58 to $2.81 \mathrm{MPa} \cdot \mathrm{m}^{1 / 2}$, while measurements for the F-CNT nanocomposite ranged from 2.04 to $2.65 \mathrm{MPa} \cdot \mathrm{m}^{1 / 2}$.

Aside from the differences in strength and fracture toughness observed between the two neat epoxy systems (explained by the different curing agents that were used), several important observations can be made. The spall strength measurements plotted in Figure 5.27 and Figure 5.28 demonstrate a clear effect of the nanotubes. While the neat epoxy has higher spall strengths than the epoxy/CNT composite at elevated shock stresses, the strengths of both materials appear to converge at lower shock stresses ( $200 \mathrm{MPa})$, providing evidence for a potential shock stress-state-activated 


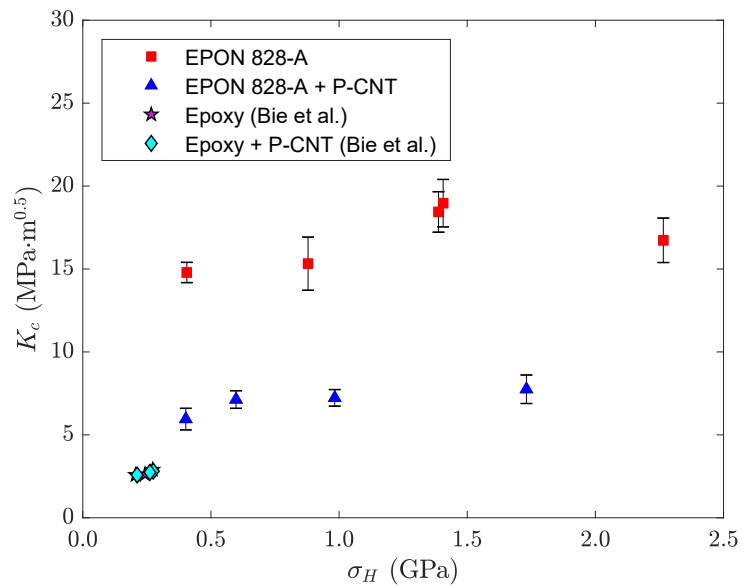

Figure 5.29: Fracture toughness measurements for neat EPON 828-A and EPON 828$\mathrm{A}+\mathrm{P}-\mathrm{CNT}$ composite plotted against Hugoniot state stress.

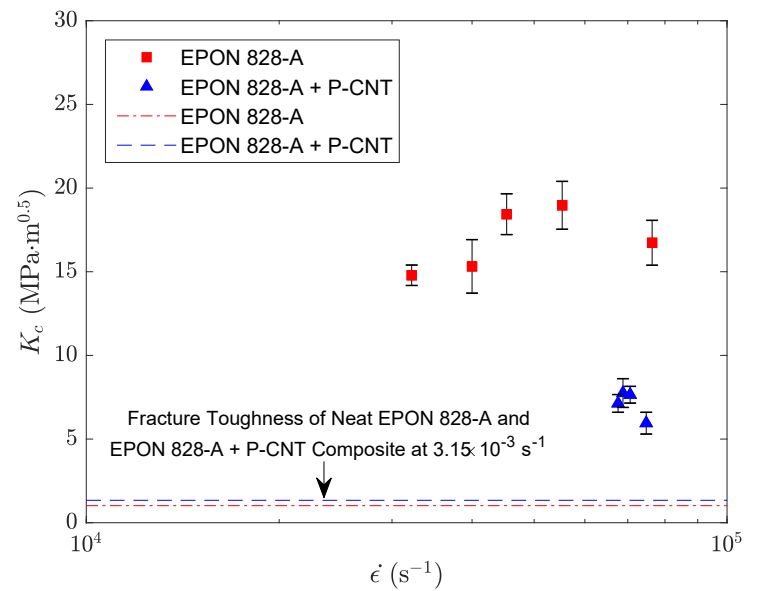

Figure 5.30: Fracture toughness measurements for neat EPON 828-A and EPON 828$\mathrm{A}+\mathrm{P}-\mathrm{CNT}$ composite at low and high strain rates.

transition between dominant failure mechanisms. The apparent convergence of the measured spall strengths at low shock stress is encouraged by the results of Bie et al. [5], who observed little difference between spall strengths measured for neat bisphenol-A epoxy and epoxy/CNT composite at shock stresses around $250 \mathrm{MPa}$. An examination of the strain rates associated with these impacts may provide a different explanation. The difference in the spall strengths measured for neat epoxy and epoxy/CNT composite at low shock stress is perhaps simply offset by the difference in tensile strain rate, where the strain rates for low-shock-stress impacts with epoxy/CNT composite were much higher than those measured for low-shock-stress impacts with the neat epoxy. Other variables that might have influenced performance (but were not tested in these experiments) include the weight fraction of the nanotubes in the composite, the aspect ratio of the nanotubes, and the use of single-walled rather than multi-walled carbon nanotubes.

Bie et al. [5] found that the F-CNT nanocomposite specimens had lower average spall strengths than the P-CNT nanocomposite specimens, indicating that functionalizing the nanotubes with ozone had a slight detrimental effect on dynamic tensile strength. This result is counterintuitive, since it was originally believed that increasing the bond strength between the nanotubes and the matrix via ozone-functionalization would increase the spall strengths. 
Functionalizing carbon sheets (graphene or nanotubes) with ozone is an established method that is intended to encourage covalent bonding between the carbon atoms in the nanotube and polar organic substances [86] (i.e., the epoxy matrix). Other strategies for functionalization exist [87] that might be better suited for improving the bond strength in epoxy/carbon nanotube composite intended for use in high-strain rate applications.

\subsubsection{Fracture Analysis}

Understanding the interactions between the carbon nanotubes and the epoxy matrix is critical for determining which fracture mechanisms played an active role in the dynamic failure of the nanocomposite. To investigate this further, a scanning electron microscope (SEM) was used to image internal fracture surfaces from fragments of a nanocomposite target recovered from a plate impact experiment. While the complete collection of SEM images can be viewed in Appendix C, two images from that collection with features of particular interest are shown here in Figures 5.31 and 5.32. A photo showing several of the nanocomposite fragments is provided in Figure 5.33.

The fracture surface shown in Figure 5.31 reveals a relatively even distribution of the randomlyoriented nanotubes throughout the epoxy matrix with few zones of agglomeration. Close inspection of the surface reveals voids left behind by several nanotubes that were pulled out during tensile failure. Failure by nanotube pull-out can also be observed on the fracture surface shown in Figure 5.32, where several nanotubes remain partially-embedded in the epoxy matrix.

Two previous investigations by Tang et al. $[88,89]$ have identified three fracture mechanisms in epoxy/carbon nanotube composite under quasi-static tensile loading. These include: nanotube pull-out, sliding fracture, and immediate fracture. SEM images presented by Bie et al. [5] showed that these fracture mechanisms are also active for epoxy/carbon nanotube composite subjected to plate impact loading. Sliding fracture, characterized by partial nanotube pull-out and subsequent nanotube fracture, is the most effective mechanism for strengthening [5]. Next is immediate frac-

ture, where only nanotube fracture occurs with no nanotube sliding, followed by nanotube pull-out, where no nanotube fracture occurs. It therefore follows that a composite material that exhibits in- 


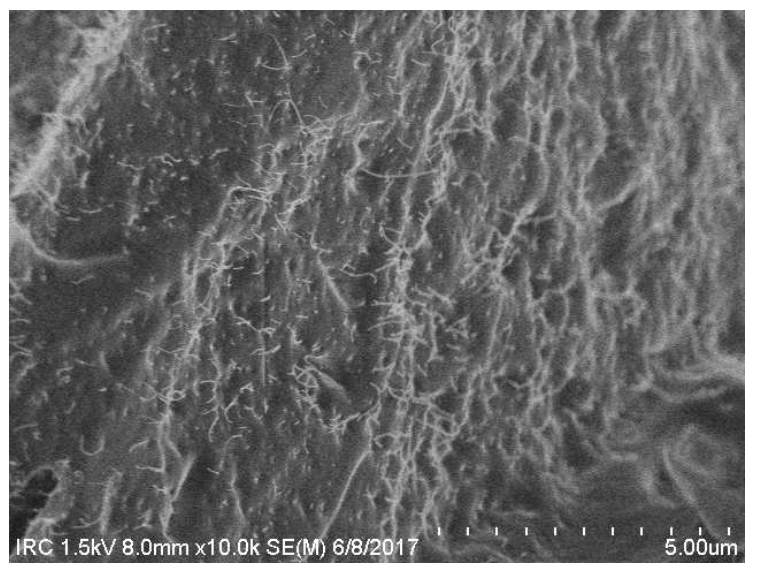

Figure 5.31: SEM of a recovered nanocomposite target showing a top-down view of a fracture surface.

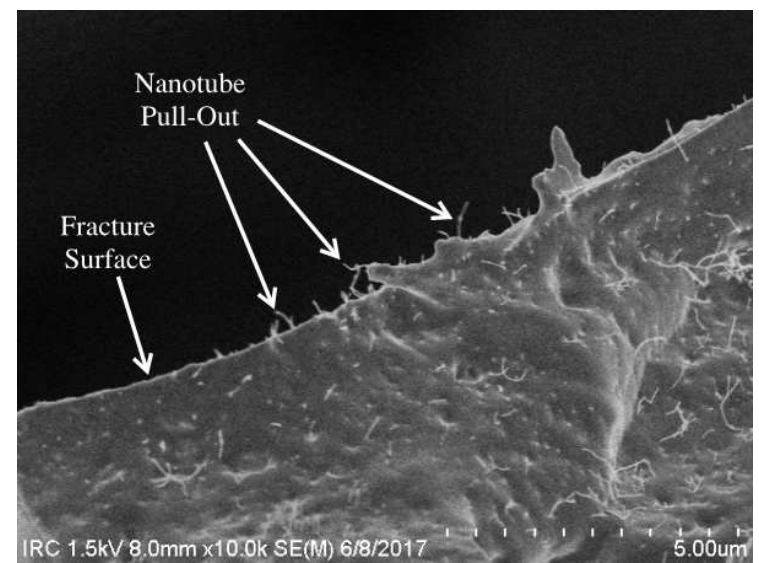

Figure 5.32: SEM of a recovered nanocomposite target showing a profile view of a fracture surface.

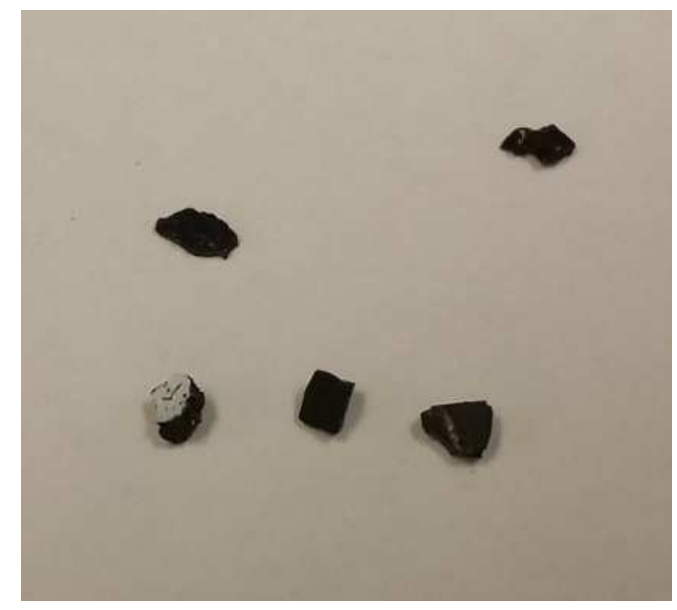

Figure 5.33: Fragments of a nanocomposite target recovered from a plate impact experiment. The free surface was spray painted with white enamel prior to impact. This made it easy to distinguish between internal fracture surfaces and the external free surface.

creased failure by sliding fracture and/or immediate fracture would be more likely to delay the onset of tensile failure under quasi-static and dynamic loading.

Examination of the fracture surfaces of the EPON 828-A + P-CNT fragments revealed that failure occurred primarily via nanotube pull-out. Not a single definitive instance of sliding fracture or immediate fracture was observed (although it is possible that they still occurred). This indicates that, rather than serving to support the large dynamic tensile loads, the nanotubes likely acted as initiation sites for spall failure between the nanotubes and the epoxy matrix. After nanotube pullout occurred, the voids left behind in the weak epoxy matrix would have acted as zones of stress 


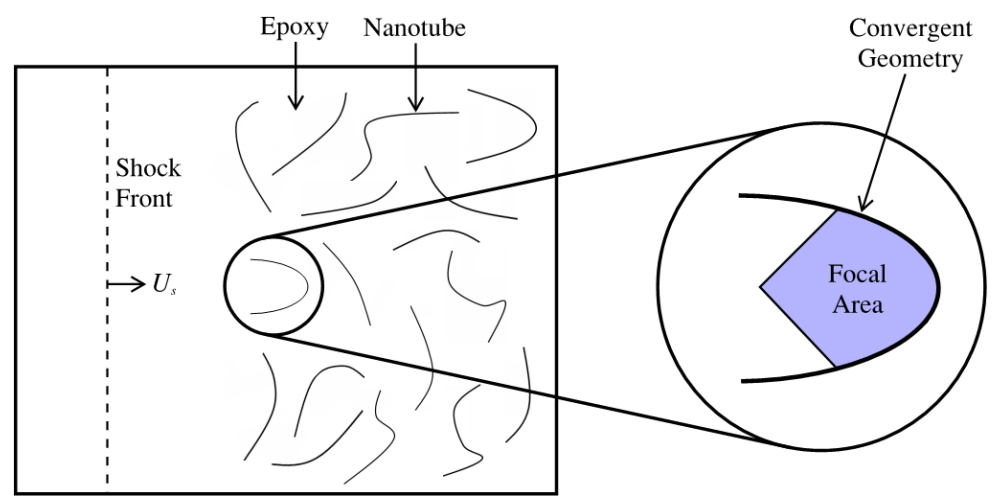

Figure 5.34: Geometry-induced shock strengthening in the focal area of a concave nanotube embedded in an epoxy matrix [92].

concentration. The holes would have grown and coalesced with further application of tensile stress, eventually resulting in catastrophic tensile failure of the bulk composite.

For the case of a nanocomposite with excellent bonds between its nanotubes and the matrix, the transfer of tensile stress from the matrix to the nanotubes would increase, resulting in a higher frequency of instances of sliding or immediate fracture. A strengthening effect would therefore be observed. This could explain why Bie et al. [5] observed no significant reduction in strength between the specimens of neat epoxy and the specimens of P-CNT composite, and why the neat EPON 828-A specimens had higher spall strengths than the EPON 828-A + P-CNT specimens.

An alternative (albeit purely speculative) explanation for the results can be found by examining the morphology of the nanotubes (as seen in Figure 5.31). Specifically, many of the nanotubes possess curved or angular geometries. Previous investigations [90-92] of 2D liquid-solid shock interactions with convergent (concave) geometries have provided evidence for a shock-strengthening effect in the focal region, resulting in cavitation at the liquid-solid interface. Knowing that cavitation in liquids caused by dynamic tensile loading is qualitatively equivalent to spall fracture in solids [7], it may be reasonable to extend that phenomenon to 2D solid-solid shock interactions with convergent geometries.

Consider the schematic shown in Figure 5.34, which depicts a zone of shock intensification created from the interaction between a planar shock and a concave nanotube embedded in an epoxy matrix. The shock impedance of carbon is greater than that of epoxy $\left(Z_{\text {carbon }}>Z_{\text {epoxy }}\right)$. The re- 
flected wave would therefore return as an intensified compressive shock wave, where the angle of the walls of the nanotube would direct the reflected shock front towards itself. The matter located at the focal region would experience a rapid jump in compressive stress and particle velocity that would be significantly higher than that of the surrounding epoxy matrix. Stress and velocity gradients would develop between the focal region and the adjacent matter, which would generate shear at the interface between the nanotube and the epoxy. If the shear stress achieved at that interface were to exceed the shear strength of the bond between the two materials, then the bond would fail and the nanotube would delaminate from the matrix. This is critical, since the fractured interface would therefore become a preferential site for void nucleation upon the application of a tensile stress. Hence, spallation would likely initiate at these "pre-failed" interfaces.

Once again, the proposed failure process is purely speculative, but if it were true, then it would explain the reduction in spall strength that is observed from the addition of nanotubes to neat epoxy. It would also explain the differences between the spall strengths reported here and those reported by Bie et al. [5], since it is possible that the epoxy used in their nanocomposite created nanotube/matrix bonds with higher dynamic strength than the EPON 828 epoxy used in this study. Future work would be necessary to prove/disprove this failure process. A series of spall experiments could be conducted with various nanocomposite materials, each using the same nanotubes, but with differing matrix materials to test for effects of surface chemistry and adhesion strength. 


\section{Conclusions}

The objective of this work was to investigate dynamic failure in a variety of materials subjected to uniaxial, high-strain-rate tensile loading, with a focus on studying the behaviour of a nanomodified polymer-matrix composite. Performance was evaluated in terms of strength and fracture toughness. Separate experiments with a load frame and a gas gun were conducted to examine failure at quasi-static and dynamic strain rates, respectively. Differences in the magnitudes of mechanical properties measured at low and high strain rates provided evidence for significant strainrate-dependency for all materials.

Spall strengths ranged from 1.34 to $1.54 \mathrm{GPa}$ in aluminum 6061-T6, and from 153 to $200 \mathrm{MPa}$ in polymethylmethacrylate (PMMA). Fracture toughnesses ranged from 35.1 to $45.6 \mathrm{MPa} \cdot \mathrm{m}^{1 / 2}$ in aluminum 6061-T6, and from 2.03 to $3.75 \mathrm{MPa} \cdot \mathrm{m}^{1 / 2}$ in PMMA at tensile strain rates on the order of $10^{4} \mathrm{~s}^{-1}$. Successful identification of the spall strengths and fracture toughnesses in these two materials validated the experimental and analytical methods used in this investigation.

Spall strengths ranged from 404 to $585 \mathrm{MPa}$ in EPON 828-A and from 339 to $397 \mathrm{MPa}$ in EPON 828-B. Fracture toughnesses ranged from 14.8 to $19.0 \mathrm{MPa} \cdot \mathrm{m}^{1 / 2}$ in EPON 828-A and from 8.95 to $11.6 \mathrm{MPa} \cdot \mathrm{m}^{1 / 2}$ in EPON 828-B at strain rates on the order of $10^{4} \mathrm{~s}^{-1}$. An effect of the curing agent on the dynamic mechanical properties, failure behaviour, and shock response of an epoxy system was identified. These results were attributed to micromechanical interactions between adjacent polymer chains, where the binding forces and equilibrium spacing were speculated to have the largest influence on failure behaviour.

The addition of pristine multi-walled carbon nanotubes to neat epoxy had a detrimental effect on dynamic mechanical performance. Spall strengths ranged from 113 to $342 \mathrm{MPa}$, while 
fracture toughnesses ranged from 5.95 to $7.75 \mathrm{MPa} \cdot \mathrm{m}^{1 / 2}$. Fracture surfaces of recovered nanocomposite fragments were imaged using a scanning electron microscope (SEM). Very few instances of nanotube agglomeration were observed in these images. Nanotube pull-out appeared to be the dominant failure mechanism, while instances of sliding and immediate fracture were not directly observed. Finally, an effect relating to nanotube morphology was used to propose a failure process under shock compression. It is believed that the interfaces between the nanotubes and the epoxy matrix acted as preferential zones for void nucleation during tensile loading. The reduction in spall strength was attributed to weak bonding at these interfaces. 


\section{References}

[1] T. H. Antoun, L. Seaman, and D. R. Curran, "Dynamic failure of materials," tech. rep., Defense Special Weapons Agency, Alexandria, 1998.

[2] M. A. Meyers and C. T. Aimone, "Dynamic fracture (spalling) of metals," Prog. Mater. Sci., vol. 28, no. 1, pp. 1-96, 1983.

[3] G. I. Kanel, S. V. Razorenov, and V. E. Fortov, Shock-wave phenomena and the properties of condensed matter. New York: Springer Science \& Business Media, 1st ed., 2004.

[4] N. H. Murray, N. K. Bourne, Z. Rosenberg, and J. E. Field, "The spall strength of alumina ceramics," J. Appl. Phys., vol. 84, no. 2, p. 734, 1998.

[5] B. X. Bie, J.-H. Han, L. Lu, X. M. Zhou, M. L. Qi, Z. Zhang, and S. N. Luo, "Dynamic fracture of carbon nanotube/epoxy composites under high strain-rate loading," Compos. Part A Appl. Sci. Manuf., vol. 68, pp. 282-288, 2015.

[6] F. Yuan, L. Tsai, V. Prakash, A. M. Rajendran, and D. P. Dandekar, "Spall strength of glass fiber reinforced polymer composites," Int. J. Solids Struct., vol. 44, no. 24, pp. 7731-7747, 2007.

[7] D. C. Erlich, D. C. Wooten, and R. C. Crewdson, "Dynamic tensile failure of glycerol," J. Appl. Phys., vol. 42, no. 13, pp. 5495-5502, 1971.

[8] T. Antoun, L. Seaman, D. R. Curran, G. I. Kanel, S. V. Razorenov, and A. V. Utkin, Spall Fracture. New York: Springer-Verlag, 1st ed., 2003.

[9] P. J. Hazell, Armour: materials, theory, and design. Boca Raton: CRC Press, 1st ed., 2015.

[10] "Hypervelocity Impact," 2013.

[11] M. Grujicic, W. C. Bell, and B. Pandurangan, "Design and material selection guidelines and strategies for transparent armor systems," Mater. Des., vol. 34, pp. 808-819, 2012.

[12] B. Hopkinson, Brittleness and ductility. Sheffield: Cambridge University Press, 1910.

[13] J. S. Rinehart, "Scabbing of metals under explosive attack: multiple scabbing," J. Appl. Phys., vol. 23, no. 11, pp. 1229-1233, 1952.

[14] B. M. Butcher, L. M. Barker, D. E. Munson, and C. D. Lundergan, "Influence of stress history on time-dependent spall in metals," AIAA J., vol. 2, no. 6, pp. 977-990, 1965. 
[15] A. L. Stevens and F. R. Tuler, "Effect of shock precompression on the dynamic fracture strength of 1020 steel and 6061-T6 aluminum," J. Appl. Phys., vol. 42, no. 13, pp. 5665$5670,1971$.

[16] A. L. Stevens, L. Davison, and W. E. Warren, "Spall fracture in aluminum monocrystals: a dislocation-dynamics approach,” J. Appl. Phys., vol. 43, no. 12, pp. 4922-4927, 1972.

[17] L. Davison and A. L. Stevens, "Continuum measures of spall damage," J. Appl. Phys., vol. 43, no. 3, pp. 988-994, 1972.

[18] L. Davison and A. L. Stevens, “Thermomechanical constitution of spalling elastic bodies," J. Appl. Phys., vol. 44, no. 2, pp. 668-674, 1973.

[19] L. E. Pope and A. L. Stevens, Wave propagation in beryllium single crystals. New York: Plenum Press, 1st ed., 1973.

[20] L. Davison, A. L. Stevens, and M. E. Kipp, "Theory of spall damage accumulation in ductile metals," J. Mech. Phys. Solids, vol. 25, no. 1, pp. 11-28, 1977.

[21] L. Davison and R. A. Graham, "Shock compression of solids," Phys. Rep., vol. 55, no. 4, pp. 255-379, 1979.

[22] D. E. Grady, "Local inertial effects in dynamic fragmentation," J. Appl. Phys., vol. 53, no. 1, pp. 322-325, 1982.

[23] B. R. Breed, C. L. Mader, and D. Venable, "Technique for the determination of dynamictensile-strength characteristics," J. Appl. Phys., vol. 38, no. 8, pp. 3271-3275, 1967.

[24] J. N. Johnson, "Dynamic fracture and spallation in ductile solids," J. Appl. Phys., vol. 52, no. 4, pp. 2812-2825, 1981.

[25] D. R. Curran, D. A. Shockey, and L. Seaman, "Dynamic fracture criteria for a polycarbonate," J. Appl. Phys., vol. 44, no. 9, pp. 4025-4038, 1973.

[26] L. Seaman, D. R. Curran, and D. A. Shockey, "Computational models for ductile and brittle fracture," J. Appl. Phys., vol. 47, no. 11, pp. 4814-4826, 1976.

[27] L. Seaman, D. R. Curran, and R. C. Crewdson, "Transformation of observed crack traces on a section to true crack density for fracture calculations," J. Appl. Phys., vol. 49, no. 10, pp. 5221-5229, 1978.

[28] D. V. Keller and J. G. Trulio, "Mechanism of spall in lucite," J. Appl. Phys., vol. 34, no. 1, pp. 172-175, 1963.

[29] A. L. Stevens and L. E. Pope, Metallurgical effects at high strain rates. New York: Springer US, 1973.

[30] D. E. Grady, "The spall strength of condensed matter," J. Mech. Phys. Solids, vol. 36, no. 3, pp. 353-384, 1988. 
[31] L. M. Barker and R. E. Hollenbach, "Shockwave studies of PMMA, fused silica , and sapphire," J. Appl. Phys., vol. 41, no. 10, pp. 4208-4226, 1970.

[32] J. L. Jordan, D. Casem, and M. Zellner, “Shock response of polymethylmethacrylate," J. Dyn. Behav. Mater., vol. 2, no. 3, pp. 372-378, 2016.

[33] W. J. Carter and S. P. Marsh, "Hugoniot equation of state of polymers," tech. rep., Los Alamos National Laboratory, Los Alamos, 1995.

[34] W. Chen, F. Lu, and M. Cheng, "Tension and compression tests of two polymers under quasistatic and dynamic loading," Polym. Test., vol. 21, no. 2, pp. 113-121, 2002.

[35] A. Faye, V. Parmeswaran, and S. Basu, "Mechanics of dynamic fracture in polycarbonate," Procedia Mater. Sci., vol. 3, pp. 1304-1309, 2014.

[36] J. C. F. Millett, E. N. Brown, G. T. Gray, N. K. Bourne, D. C. Wood, and G. Appleby-Thomas, "The effects of changing chemistry on the shock response of basic polymers," J. Dyn. Behav. Mater, vol. 2, no. 3, pp. 326-336, 2016.

[37] J. C. F. Millett, N. K. Bourne, and N. R. Barnes, "The behavior of an epoxy resin under one-dimensional shock loading," J. Appl. Phys., vol. 92, no. 11, pp. 6590-6594, 2002.

[38] J. C. F. Millett and N. K. Bourne, "The deviatoric response of polymethylmethacrylate to one-dimensional shock loading," J. Appl. Phys., vol. 88, no. 12, pp. 7037-7040, 2000.

[39] N. A. Razack and L. A. Varghese, "The effect of various hardeners on the mechanical and thermal properties of epoxy resin," Int. J. Eng. Reseach Technol., vol. 3, no. 1, pp. 2662-2665, 2014.

[40] R. Jain, P. Kukreja, A. K. Narula, and V. Chaudhary, "Studies of the curing kinetics and thermal stability of epoxy resins using a mixture of amines and anhydrides," J. Appl. Polym. Sci., vol. 100, no. 5, pp. 3919-3925, 2006.

[41] D. E. Munson and R. P. May, "Dynamically determined high-pressure compressibilities of three epoxy resin systems," J. Appl. Phys., vol. 43, no. 3, pp. 962-971, 1972.

[42] V. K. Golubev, S. A. Novikov, and Y. S. Sobolev, "Effect of temperature on spall of polymer materials," J. Appl. Mech. Tech. Phys., vol. 23, no. 1, pp. 134-141, 1982.

[43] I. P. Parhomenko and A. V. Utkin, Matter under extreme conditions. Moscow: IVTAN, 1990.

[44] T. R. Guess, "Some dynamic mechanical properties of an epoxy," tech. rep., Sandia Laboratories, Albuquerque, 1968.

[45] E. Zaretsky, G. DeBotton, and M. Perl, "The response of a glass fibers reinforced epoxy composite to an impact loading," Int. J. Solids Struct., vol. 41, no. 2, pp. 569-584, 2004.

[46] J. C. F. Millett, N. K. Bourne, Y. J. E. Meziere, R. Vignjevic, and A. A. Lukyanov, "The effect of orientation on the shock response of a carbon fibre-epoxy composite," Compos. Sci. Technol., vol. 67, no. 15-16, pp. 3253-3260, 2007. 
[47] A. A. Lukyanov, "An equation of state of a carbon-fibre epoxy composite under shock loading," Eur. Phys. J. B, vol. 74, no. 1, pp. 35-45, 2010.

[48] S. M. Peiris and G. J. Piermarini, Static compression of energetic materials. Berlin: SpringerVerlag, 1st ed., 2008.

[49] J. C. F. Millett and N. K. Bourne, "Shock response of the elastomer, polychloroprene," J. Appl. Phys., vol. 89, no. 5, pp. 2576-2579, 2001.

[50] X. Xiao, "Dynamic tensile testing of plastic materials," Polym. Test., vol. 27, no. 2, pp. 164178, 2008.

[51] F. E. Gunawan, "Dynamic debonding strength of fiber glass composite," Procedia Eng., vol. 10, pp. 25-30, 2011.

[52] P. W. Cooper, Explosives engineering. New York: Wiley-VCH, 4th ed., 2002.

[53] M. Rice, R. McQueen, and J. Walsh, "Compression of solids by strong shock waves," Solid State Phys., vol. 6, pp. 1-63, 1958.

[54] L. E. Kinsler, A. R. Frey, A. B. Coppens, and J. V. Sanders, Fundamentals of acoustics, vol. 1. New York: Wiley-VCH, 4 ed., 1999.

[55] P. Chevrier and J. R. Klepaczko, "Spall fracture: mechanical and microstructural aspects," Eng. Fract. Mech., vol. 63, no. 3, pp. 273-294, 1999.

[56] G. I. Kanel, "Spall fracture: methodological aspects, mechanisms and governing factors," Int. J. Fract., vol. 163, no. 1, pp. 173-191, 2010.

[57] M. A. Meyers, Dynamic behavior of materials. New York: Wiley-InterScience, 1st ed., 1992.

[58] S. A. Novikov, I. I. Divinov, and A. G. Ivanov, "Failure of steel, aluminum and copper under explosive shock loading," Phys. Met. Metallogr., vol. 21, no. 4, pp. 122-128, 1966.

[59] G. V. Stepanov, "Spall fracture of metals by elastic-plastic loading waves," Probl. Prochnosti, vol. 8, pp. 66-70, 1976.

[60] S. N. Luo, Q. An, T. C. Germann, and L. B. Han, "Shock-induced spall in solid and liquid $\mathrm{Cu}$ at extreme strain rates," J. Appl. Phys., vol. 106, no. 1, p. 013502, 2009.

[61] Arkema Inc., "Plexiglas G," tech. rep., Arkema Inc., 2013.

[62] T. Weerasooriya, P. Moy, D. Casem, and W. Chen, "Fracture toughness for PMMA as a function of loading rate," in SEM Annu. Conf. Expo. Exp. Appl. Mech., (St. Louis), 2006.

[63] A. J. Kinloch, R. D. Mohammed, A. C. Taylor, C. Eger, S. Sprenger, and D. Egan, "The effect of silica nano particles and rubber particles on the toughness of multiphase thermosetting epoxy polymers," J. Mater. Sci., vol. 40, no. 18, pp. 5083-5086, 2005.

[64] L. Wu, S. V. Hoa, and M.-T. Ton-That, "Effects of composition of hardener on the curing and aging for an epoxy resin system," J. Appl. Polym. Sci., vol. 99, no. 2, pp. 580-588, 2006. 
[65] M. G. Stout, D. A. Koss, C. Liu, and J. Idasetima, "Damage development in carbon/epoxy laminates under quasi-static and dynamic loading," Compos. Sci. Technol., vol. 59, no. 16, pp. 2339-2350, 1999.

[66] D. P. Dandekar, C. A. Hall, L. C. Chhabildas, and W. D. Reinhart, "Shock response of a glassfiber-reinforced polymer composite,” Compos. Struct., vol. 61, no. 1-2, pp. 51-59, 2003.

[67] R. E. Setchell and M. U. Anderson, "Shock-compression response of an alumina-filled epoxy," J. Appl. Phys., vol. 97, no. 8, p. 083518, 2005.

[68] P. E. Lopes, F. V. Hattum, C. Pereira, P. Nóvoa, S. Forero, F. Hepp, and L. Pambaguian, "High CNT content composites with CNT buckypaper and epoxy resin matrix: impregnation behaviour composite production and characterization," Compos. Struct., vol. 92, no. 6, pp. 1291-1298, 2010.

[69] M. H. Pol and G. Liaghat, "Investigation of the high velocity Impact behavior of nanocomposites," Polym. Compos., vol. 37, no. 4, pp. 1173-1179, 2016.

[70] Glemco Inc., “6061-T6 aluminum,” tech. rep., Glemco Inc., 2016.

[71] O. T. Strand, D. R. Goosman, C. Martinez, and T. L. Whitworth, "Compact system for highspeed velocimetry using heterodyne techniques," Rev. Sci. Instrum., vol. 77, no. 8, p. 083108, 2006.

[72] S. P. Marsh, LASL shock hugoniot data. Berkeley: Univerisity of California Press, 1980.

[73] F. J. MacMaster, K. S. Chan, S. C. Bergsma, and M. E. Kassner, “Aluminum alloy 6069 part II : Fracture toughness of 6061-T6 and 6069-T6," Mater. Sci. Eng. A, vol. 289, no. 1-2, pp. 54-59, 2000.

[74] C. Rubio-Gonzalez, J. L. Ocana, G. Gomez-Rosas, C. Molpeceres, M. Paredes, A. Banderas, J. Porro, and M. Morales, "Effect of laser shock processing on fatigue crack growth and fracture toughness of 6061-T6 aluminum alloy," Mater. Sci. Eng. A, vol. 386, no. 1-2, pp. 291-295, 2004.

[75] K. G. Hoge, "Influence of strain rate on mechanical properties of 6061-T6 aluminum under uniaxial and biaxial states of stress," Exp. Mech., vol. 6, no. 4, pp. 204-211, 1966.

[76] A. Manes, L. Peroni, M. Scapin, and M. Giglio, "Analysis of strain rate behavior of an Al 6061 T6 alloy," Procedia Eng., vol. 10, pp. 3477-3482, 2011.

[77] J. A. Gorman, D. S. Wood, and T. Vreeland, "Mobility of dislocations in aluminum," J. Appl. Phys., vol. 10, no. 2, pp. 833-841, 1969.

[78] C. Kitten, Introduction to Solid State Physics. Danvers: John Wiley \& Sons, 8 ed., 2005.

[79] O. E. Petel and A. J. Higgins, "Shock wave propagation in dense particle suspensions," J. Appl. Phys., vol. 108, no. 11, p. 114918, 2010. 
[80] G. Prudhomme, P. Mercier, and L. Berthe, "PDV experiments on shock-loaded particles," in J. Phys. Conf. Ser., vol. 500, (Seattle), p. 142027, 2014.

[81] C. D. Lundergan and D. S. Drumheller, "Propagation of stress waves in a laminated plate composite," J. Appl. Phys., vol. 42, no. 2, pp. 669-675, 1971.

[82] J. Richeton, S. Ahzi, F. C. Jiang, and R. R. Adharapurapu, "Influence of temperature and strain rate on the mechanical behavior of three amorphous polymers: characterization and modeling of the compressive yield stress," Int. J. Solids Struct., vol. 43, no. 7-8, pp. 23182335, 2006.

[83] A. D. Mulliken and M. C. Boyce, "Mechanics of the rate-dependent elastic plastic deformation of glassy polymers from low to high strain rates," Int. J. Solids Struct., vol. 43, no. 5, pp. 1331-1356, 2006.

[84] T. Ree and H. Eyring, "Theory of non-Newtonian flow. I. solid plastic system," J. Appl. Phys., vol. 26, no. 7, pp. 793-800, 1955.

[85] O. E. Petel, D. L. Frost, A. J. Higgins, and S. Ouellet, "Shock-induced formation of a disordered solid from a dense particle suspension," Phys. Rev. E, vol. 85, no. 2, p. 021401, 2012.

[86] S. Huh, J. Park, Y. S. Kim, K. S. Kim, B. H. Hong, and J.-m. Nam, “UV/ozone-oxidized large-scale graphene platform with large chemical enhancement in surface- enhanced raman scattering," ACS Nano, vol. 5, no. 12, pp. 9799-9806, 2011.

[87] V. Georgakilas, M. Otyepka, A. B. Bourlinos, V. Chandra, N. Kim, K. C. Kemp, P. Hobza, R. Zboril, and K. S. Kim, "Functionalization of graphene: covalent and non-covalent approaches, derivatives and applications," Chem. Rev., vol. 112, pp. 6156-6214, 2012.

[88] L.-C. Tang, H. Zhang, X.-P. Wu, and Z. Zhang, "A novel failure analysis of multi-walled carbon nanotubes in epoxy matrix," Polymer (Guildf)., vol. 52, no. 9, pp. 2070-2074, 2011.

[89] L.-C. Tang, H. Zhang, J.-H. Han, X.-P. Wu, and Z. Zhang, "Fracture mechanisms of epoxy filled with ozone functionalized multi-wall carbon nanotubes," Compos. Sci. Technol., vol. 72, no. 1, pp. 7-13, 2011.

[90] R. E. Setchell, E. Storm, and B. Sturtevant, "An investigation of shock strengthening in a conical convergent channel,” J. Fluid Mech., vol. 56, no. 3, pp. 505-522, 1972.

[91] C. Bond, D. J. Hill, D. I. Meiron, and P. E. Dimotakis, "Shock focusing in a planar convergent geometry: experiment and simulation,” J. Fluid Mech., vol. 641, pp. 297-333, 2009.

[92] V. Eliasson, M. Mello, A. J. Rosakis, and P. E. Dimotakis, "Experimental investigation of converging shocks in water with various confinement materials," Shock Waves, vol. 20, no. 5, pp. 395-408, 2010. 


\section{Appendix A}

\section{Matlab Code}

\section{A.1 PDV Data Processing Script}

This MATLAB code was used to process and view the raw PDV data obtained from the plate impact experiments with a moving-window FFT and print spectrograms for use during the free surface velocity analysis. Original script written by Justin Huneault.

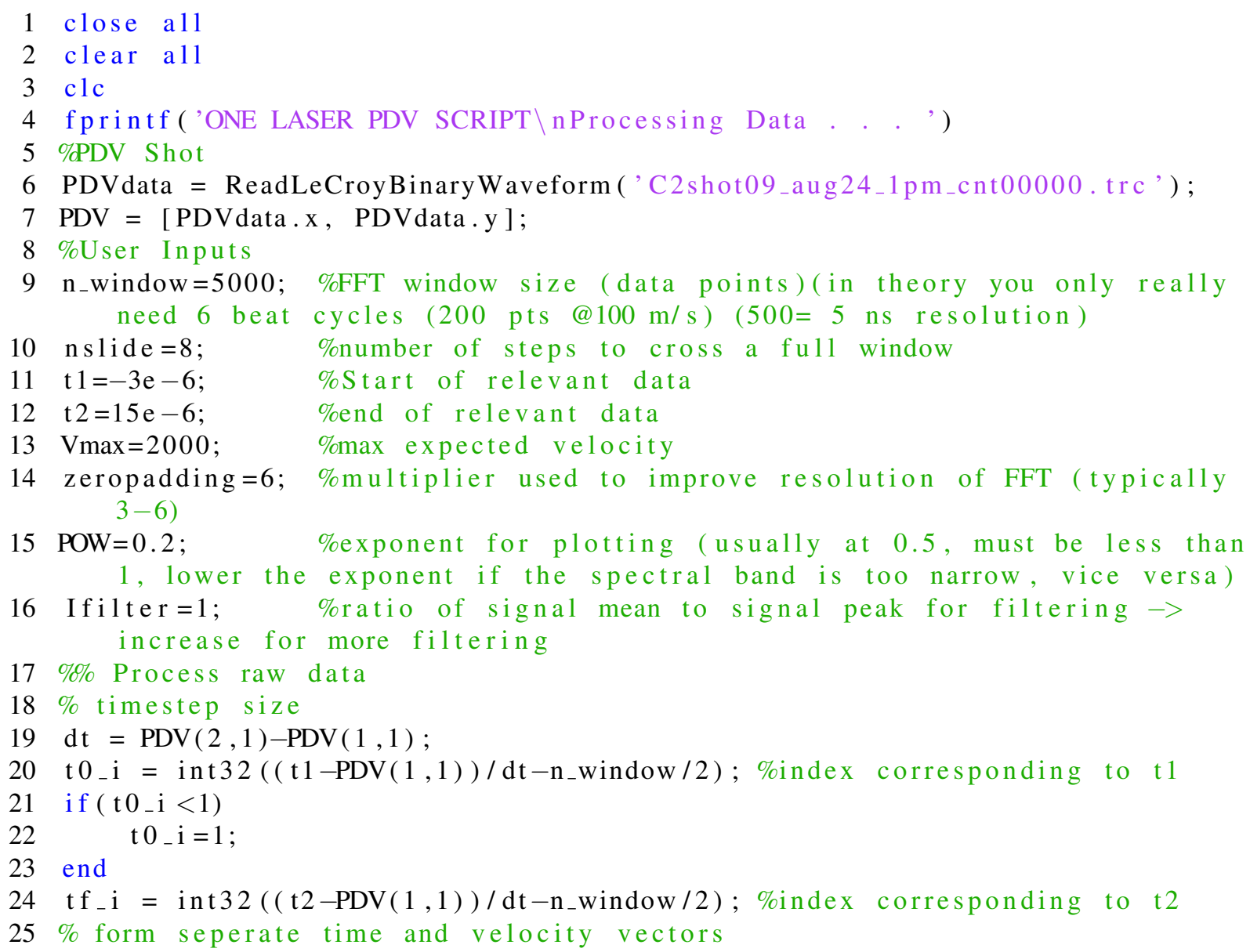




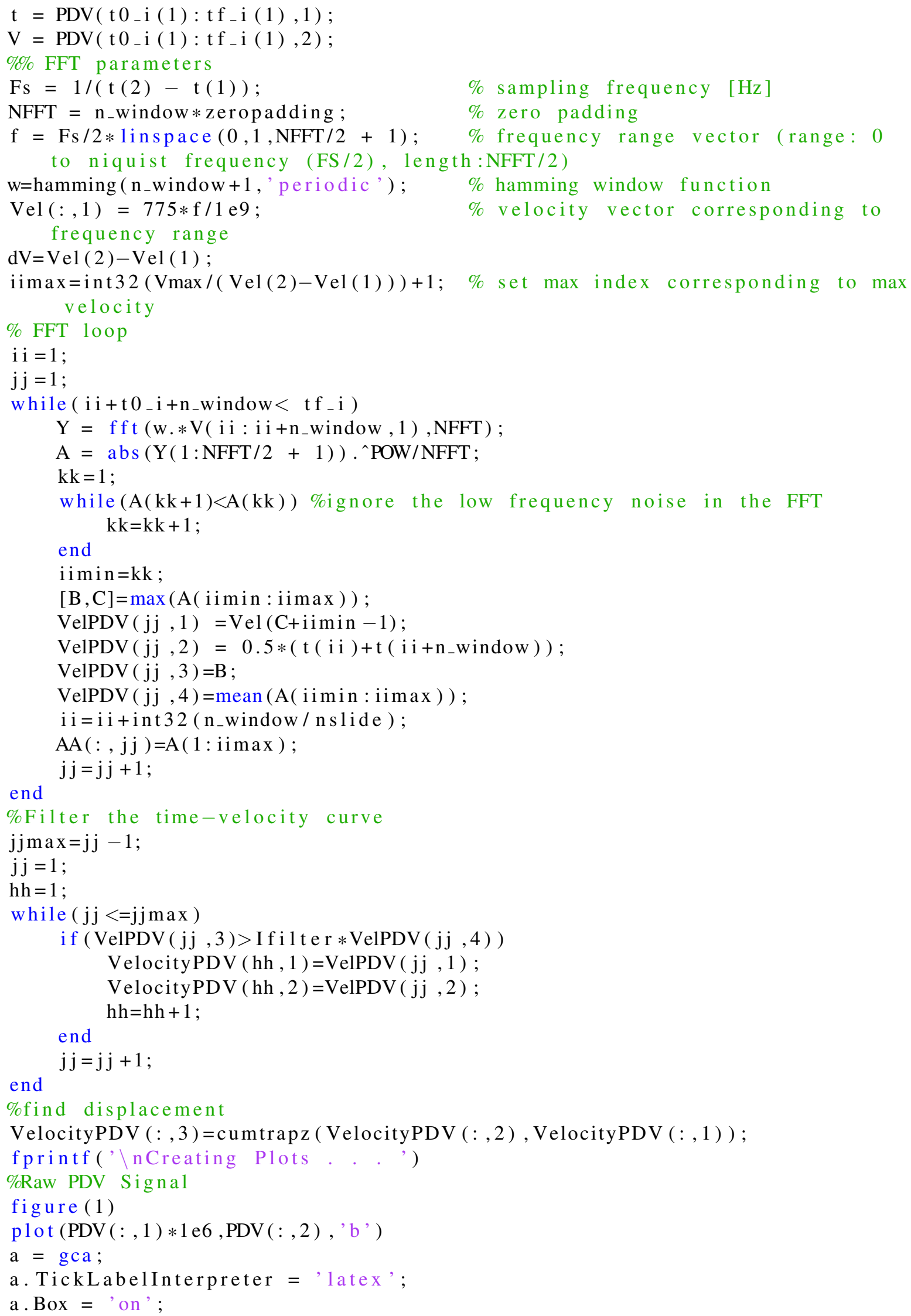




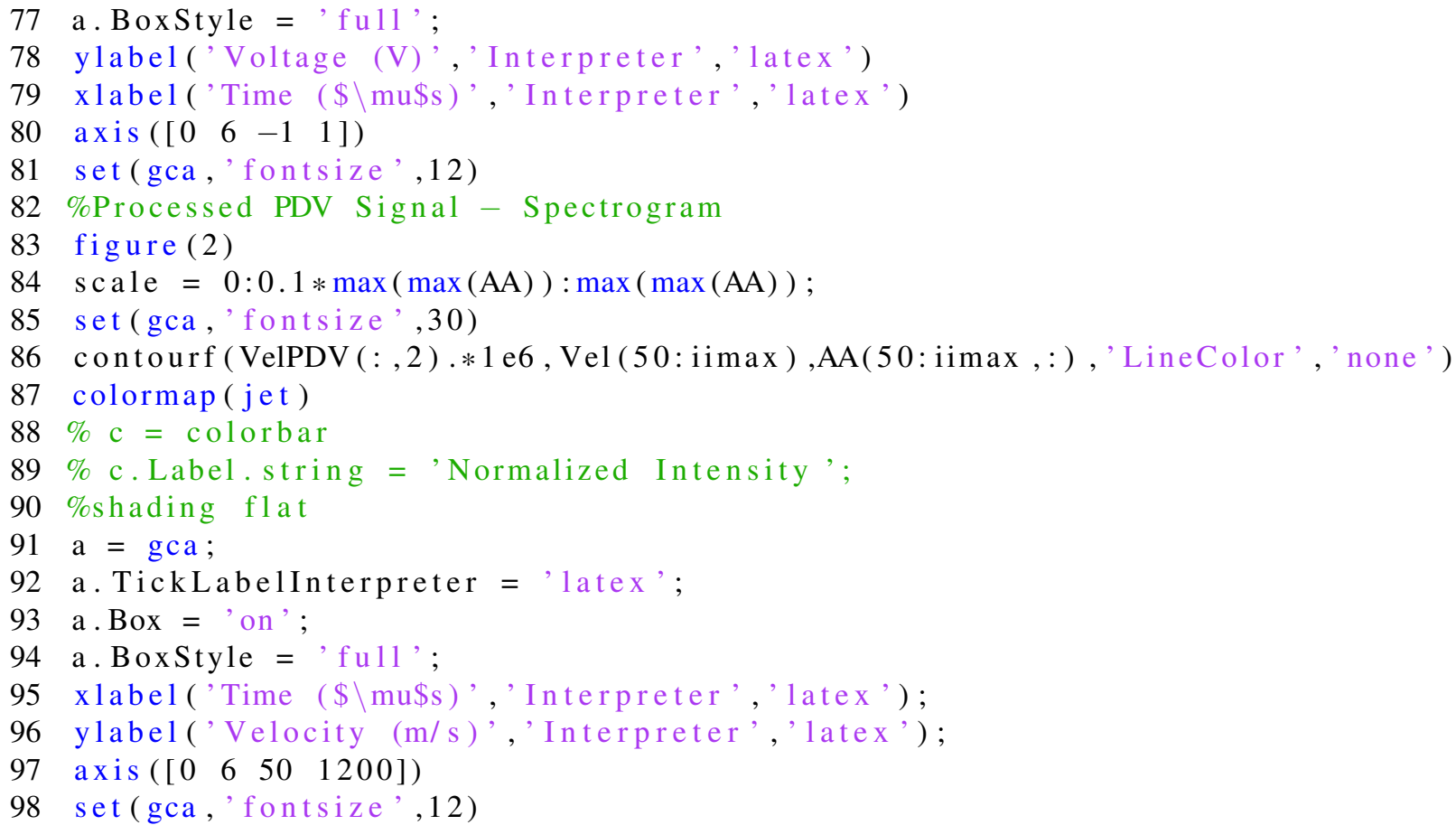

\section{A.2 Read LeCroy Binary Wave Waveform Script}

This MATLAB code was called as a function by the "PDV Data FFT Processing Script" while processing the raw PDV data obtained from the plate impact experiments. Original script provided by Justin Huneault.

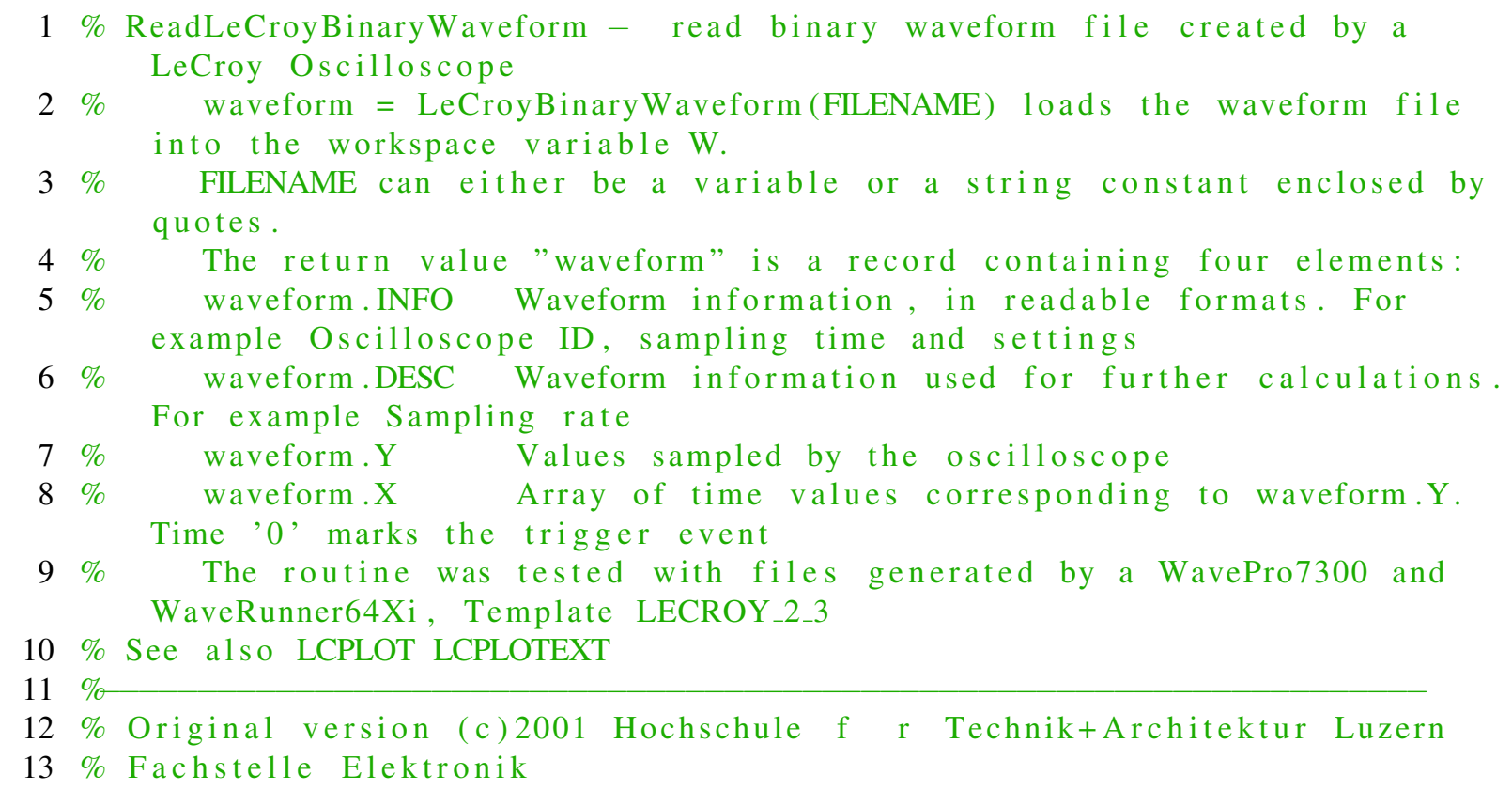


$14 \% 6048$ Horw, Switzerland

$15 \%$ Slightly modified by Alan Blankman, LeCroy Corporation, 2006

$16 \%$ July 2007; added decoding of TrigTime_array so that sequence mode waveforms decode correctly

$17 \%$ Slightly modified by Jean-Daniel Deschnes, Universite Laval, 2010

$18 \%$ Added support for sequence mode, even if RECORD_TYPE says "single_sweep"

$19 \%$ (seen on a WavePro $7 \mathrm{Zi}$ ). Output will be a MxN matrix, where $\mathrm{M}$ is the

$20 \%$ number of points in each segment, and $N$ is the number of segments. X

$21 \%$ vector also update to include offset of each trigger.

22 function wave=ReadLeCroyBinaryWaveform ( f n )

$23 \%$

$=$ WAVEDESC $+36 ; \%$ length of the descriptor block $=$ WAVEDESC+ 40; $\%$ length of the usertext

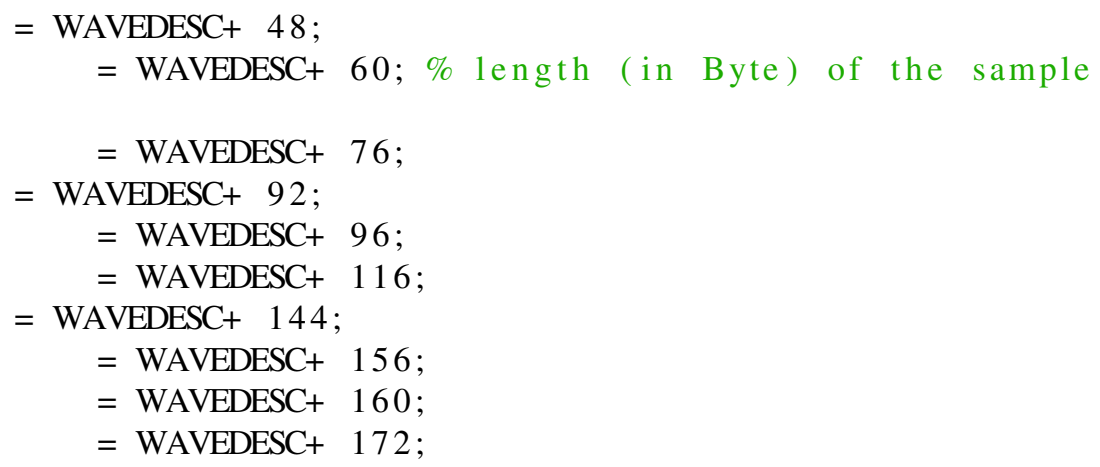




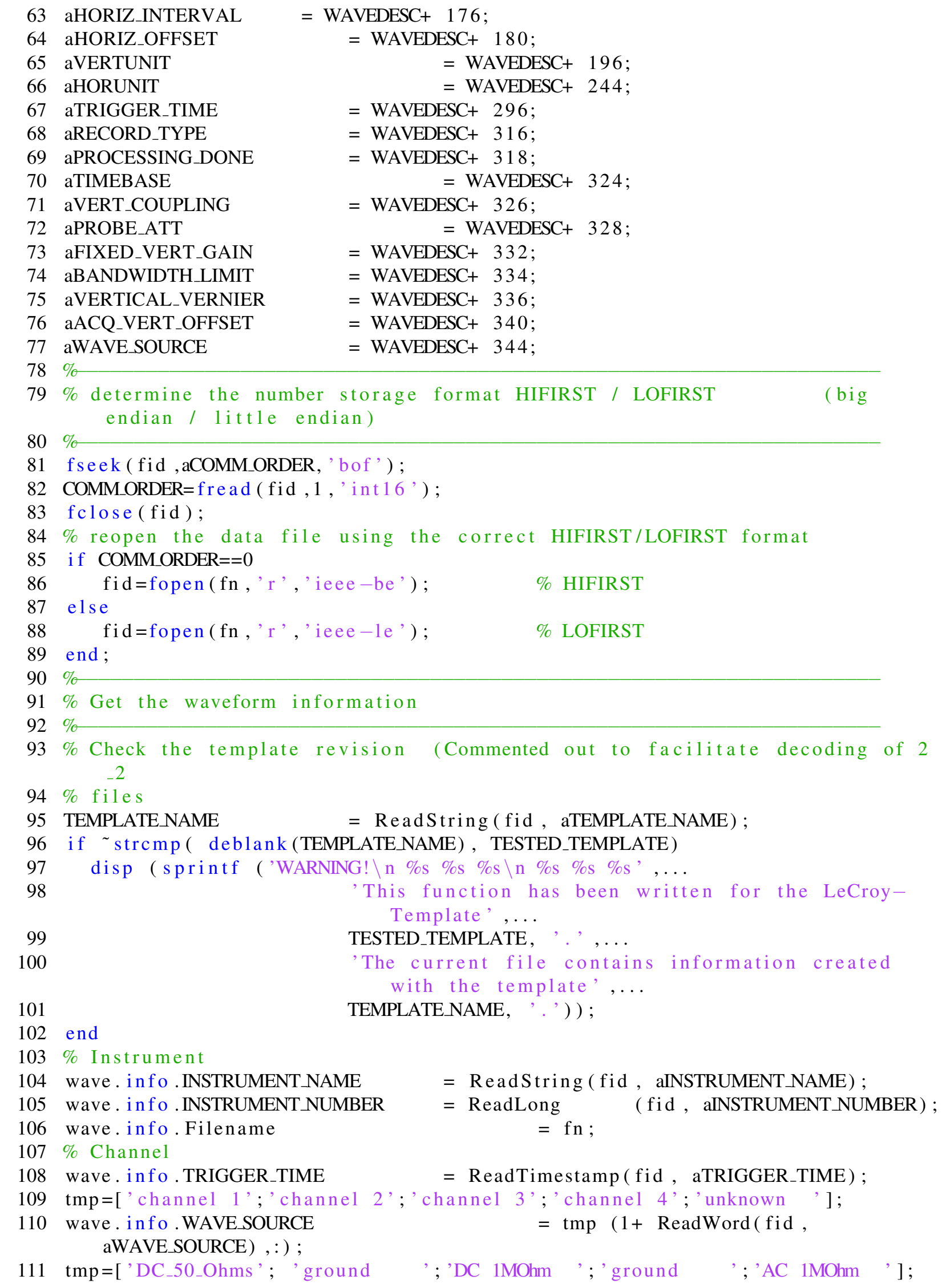




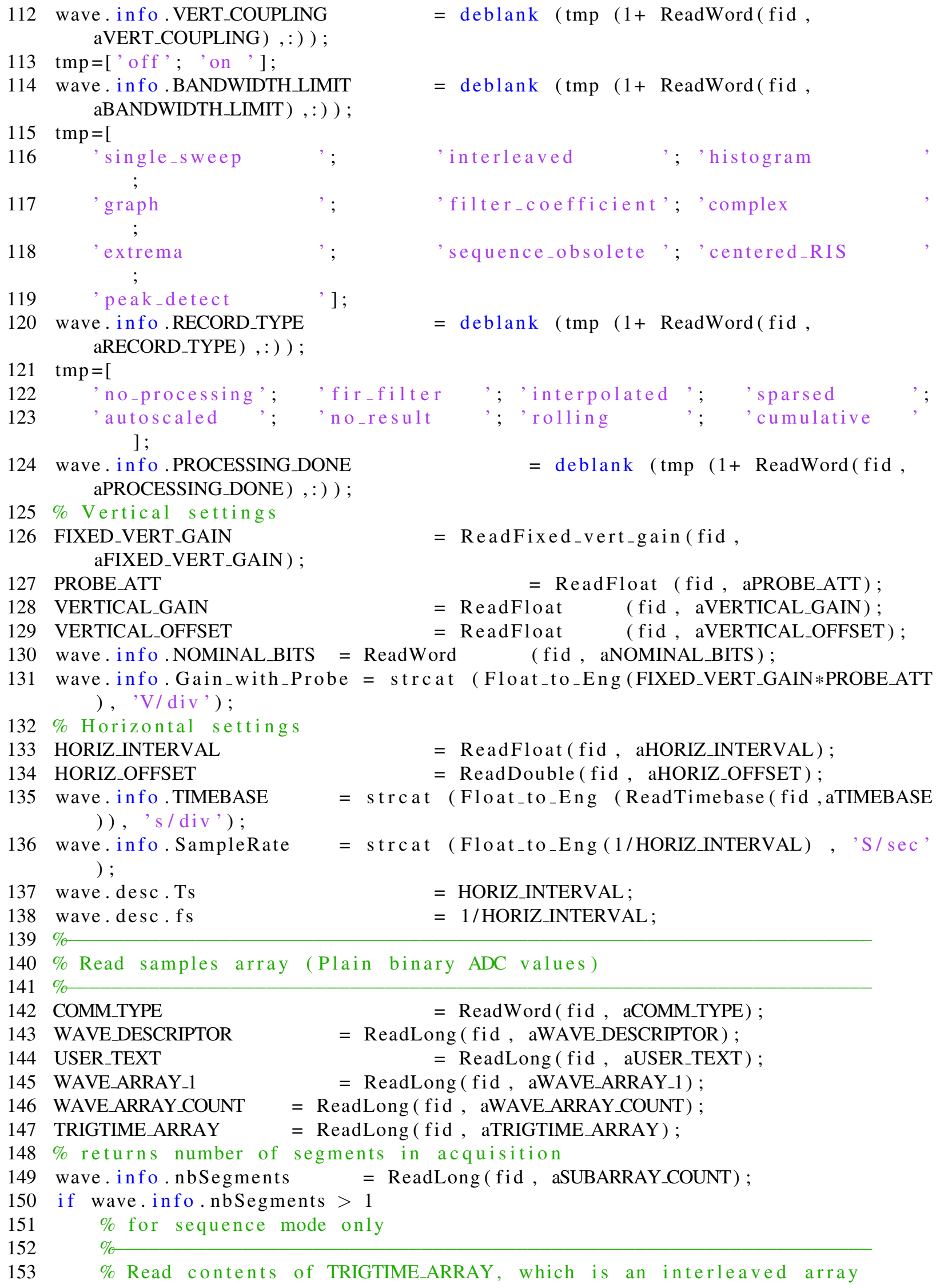




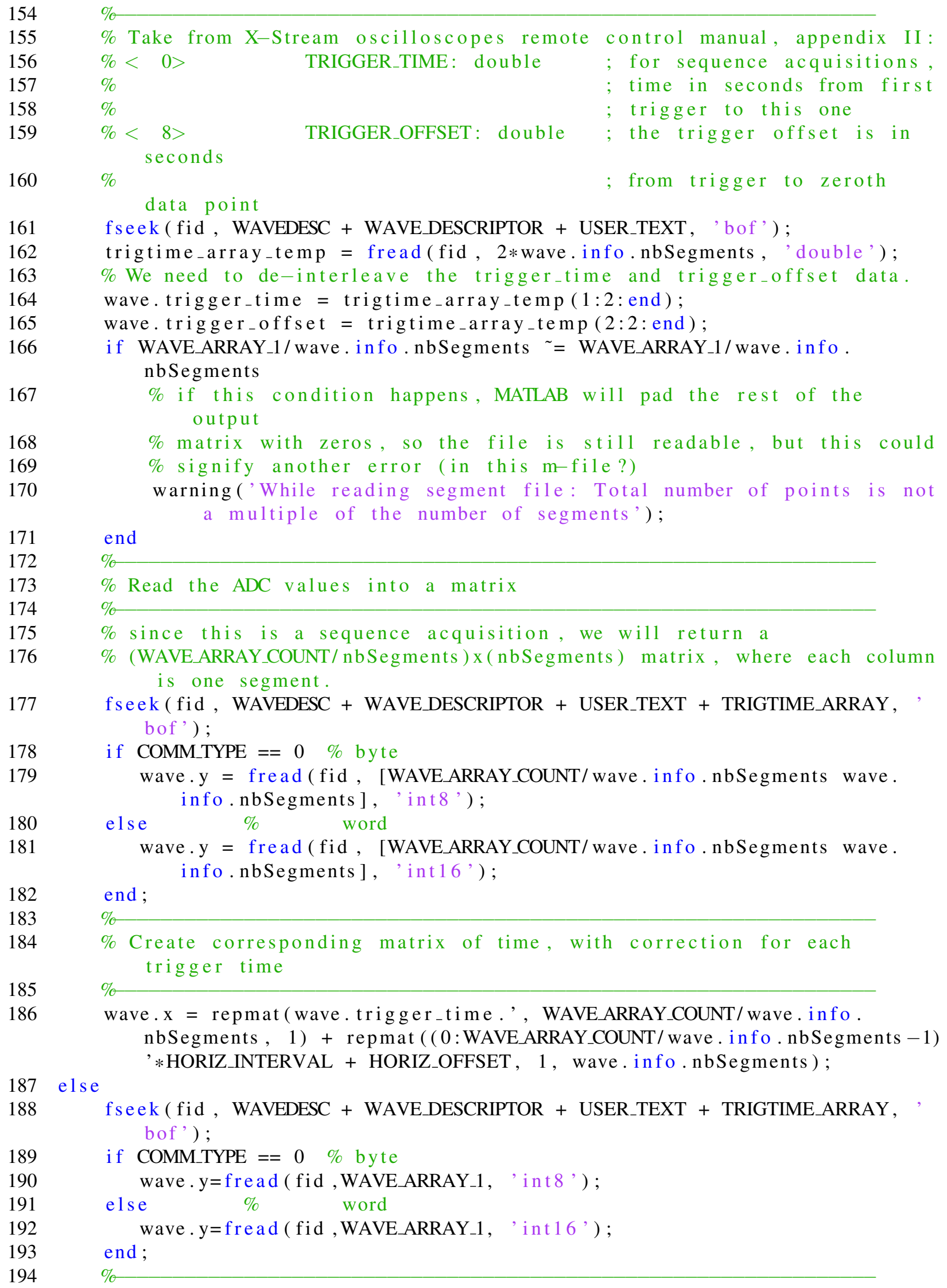




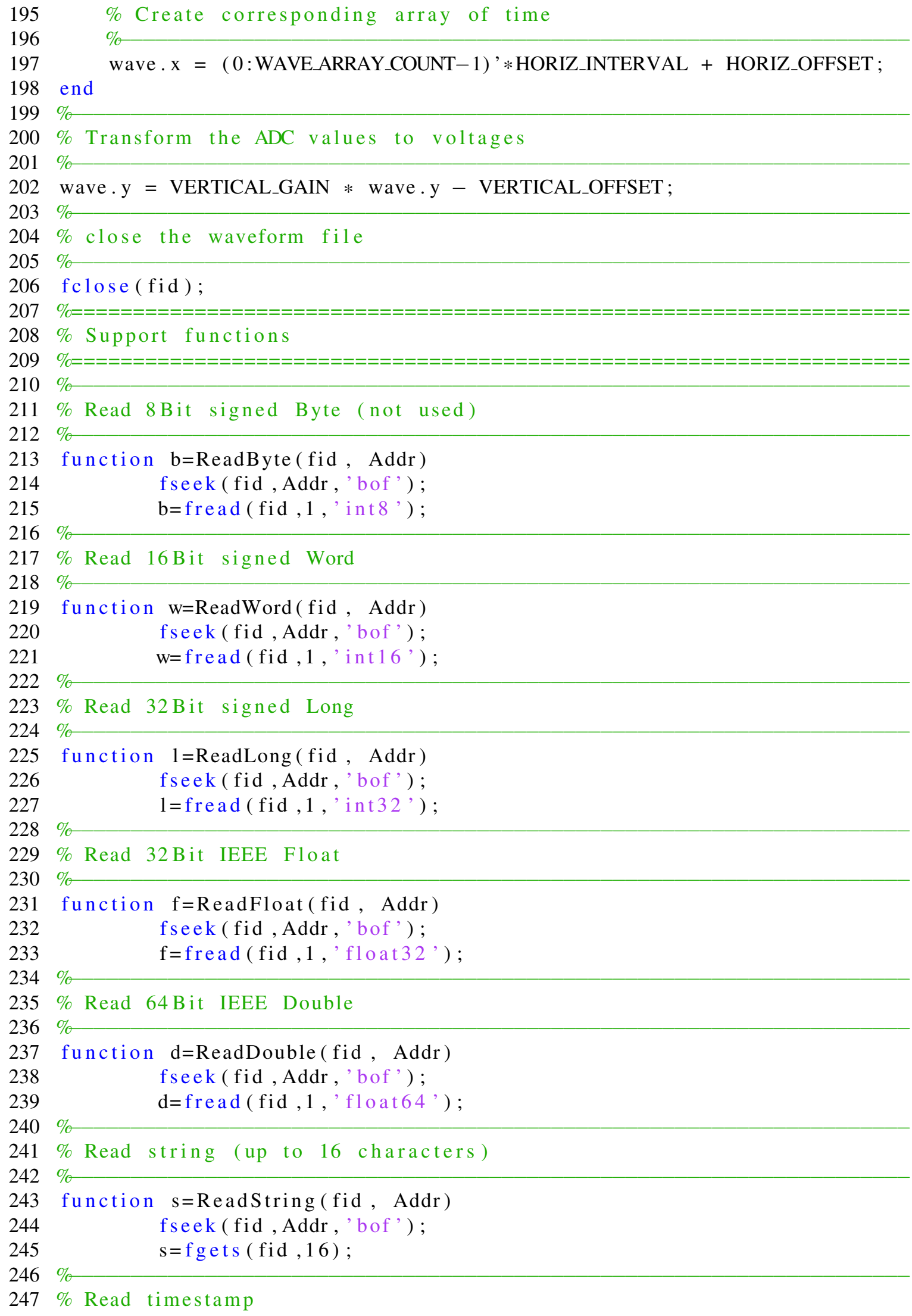

$247 \%$ Read timestamp

248 
258

259

260

261

262

263

264

265

266

267

268

269

270

271

272

273

274

275

276

277

278

279

280

281

282

283

284

285

286

287

288

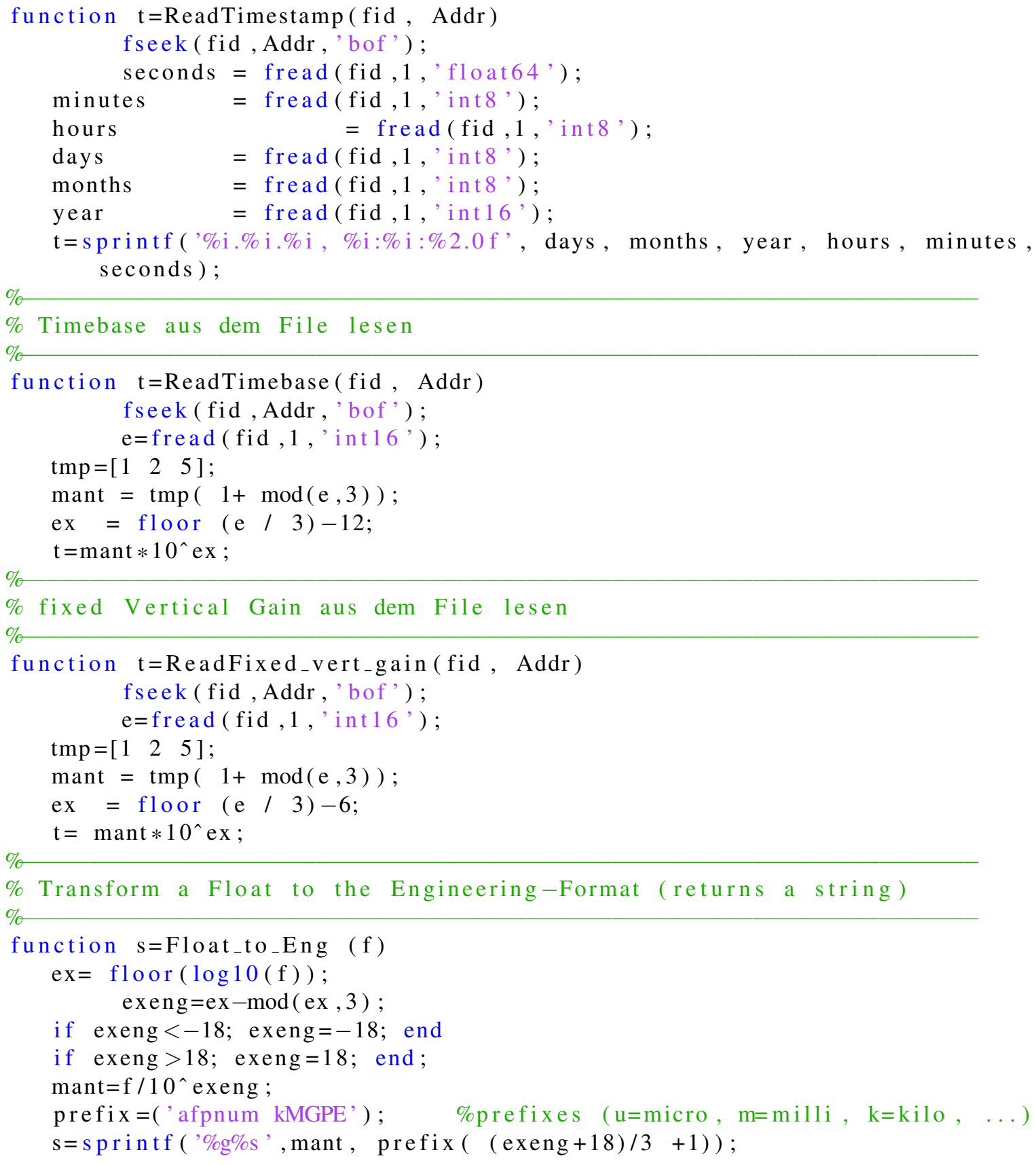

\section{A.3 Shock Pin Data Viewing Script}

This MATLAB code was used to view the raw shock pin data obtained from the plate impact experiments. Original script written by Oren E. Petel.

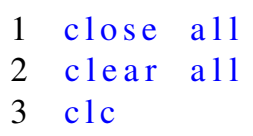




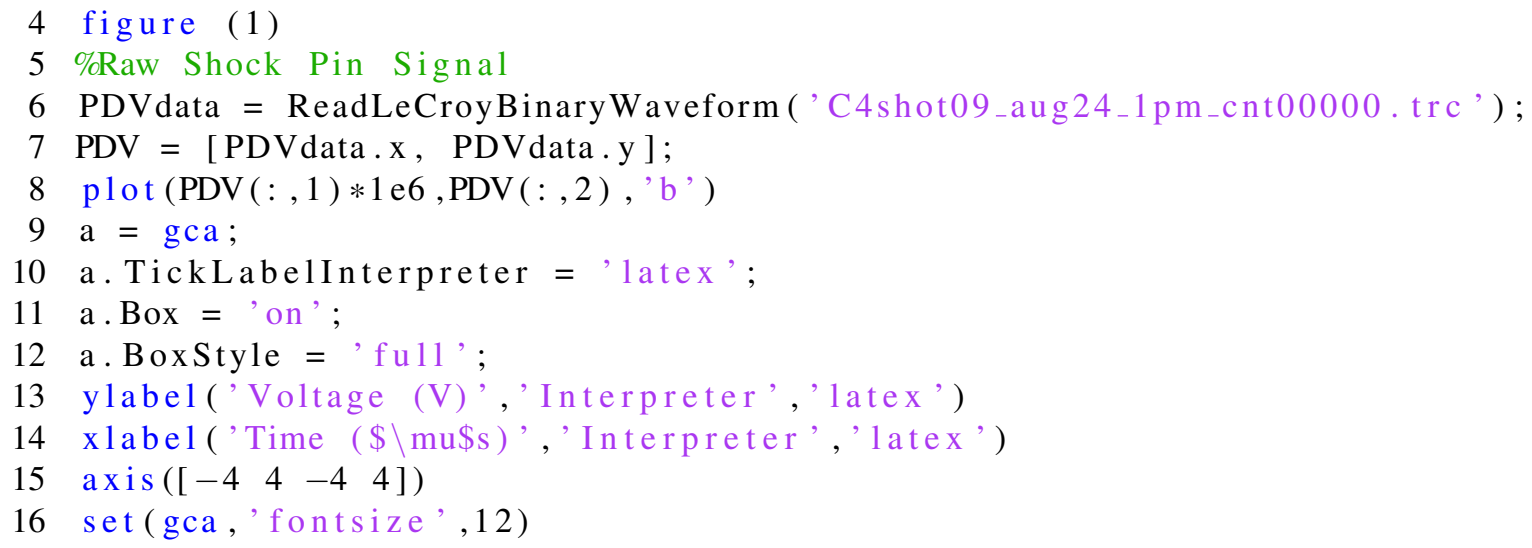

\section{A.4 Coil Gauge Data Viewing Script}

This MATLAB code was used to view the raw coil gauge data obtained from the plate impact experiments. Original script written by Oren E. Petel.

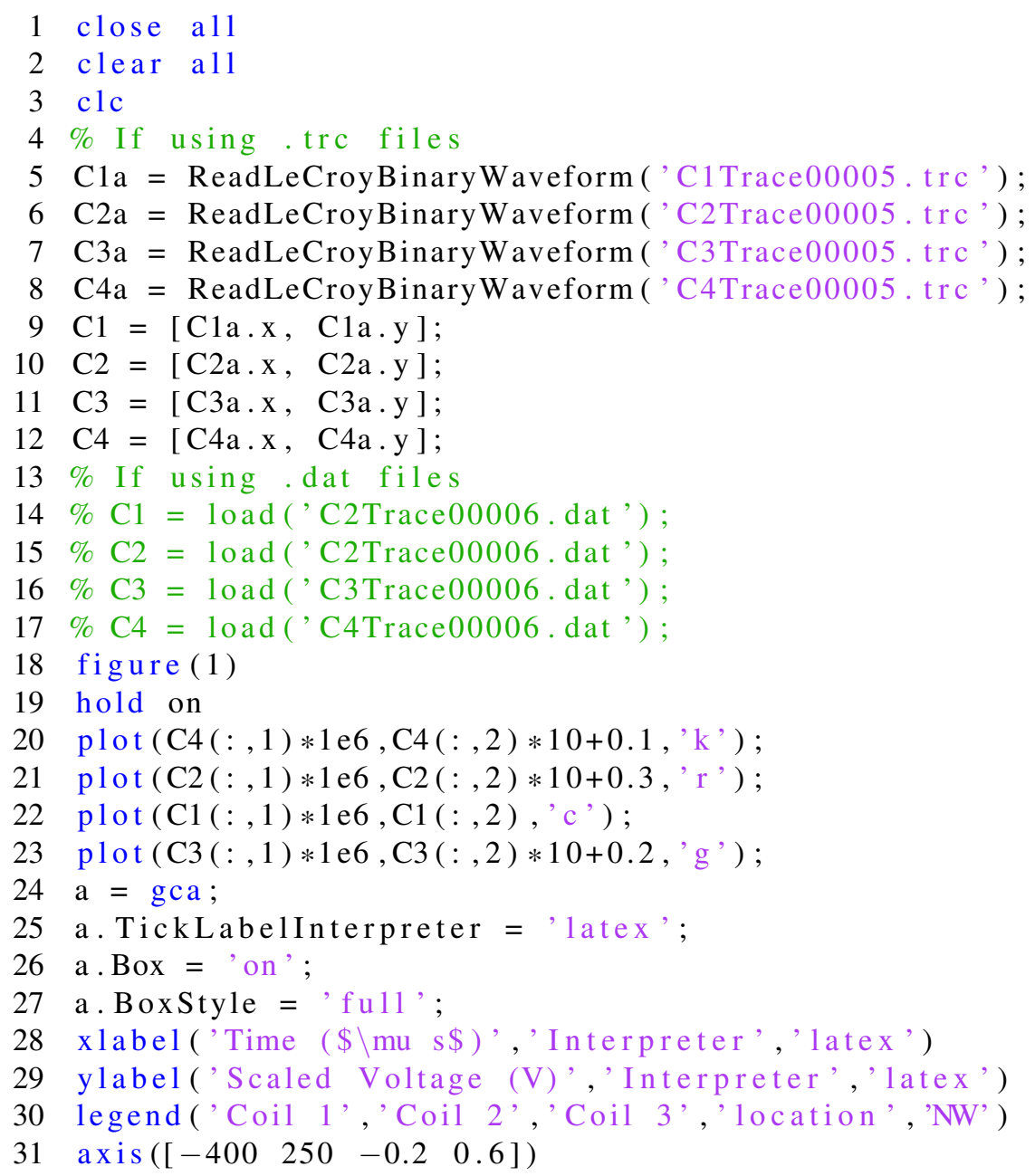


32 set(gca, 'fontsize', 12)

33 hold off 


\section{Appendix B}

\section{Spectrogram Collection}

This appendix contains all the free surface spectrograms obtained from the plate impact experiments. Each spectrogram is associated with a specific shot carried out with the single-stage light gas gun. The impact conditions of each shot are described in the figure captions.

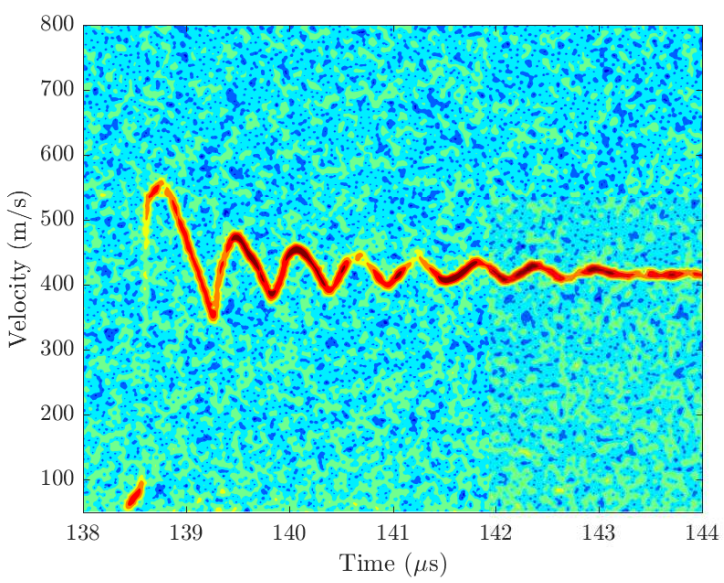

Figure B.1: An aluminum 6061-T6 target impacted with an aluminum 6061-T6 flyer plate at a velocity of $580 \mathrm{~m} \cdot \mathrm{s}^{-1}$.

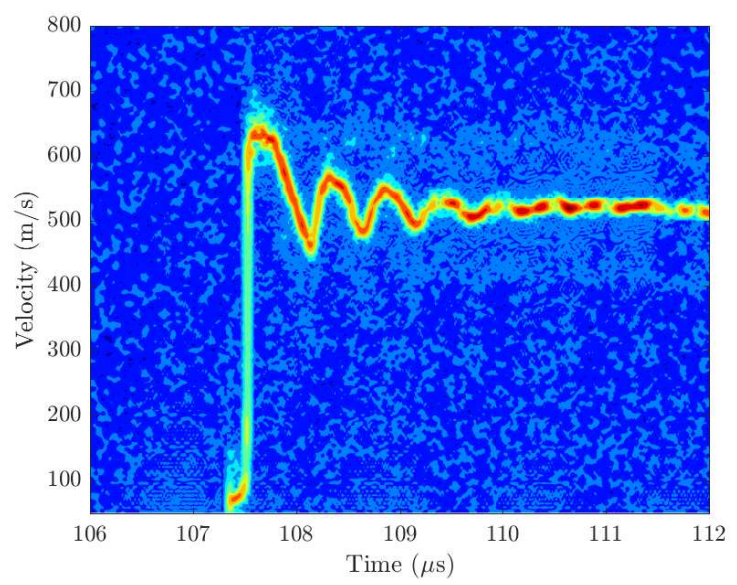

Figure B.2: An aluminum 6061-T6 target impacted with an aluminum 6061-T6 flyer plate at a velocity of $633 \mathrm{~m} \cdot \mathrm{s}^{-1}$. 


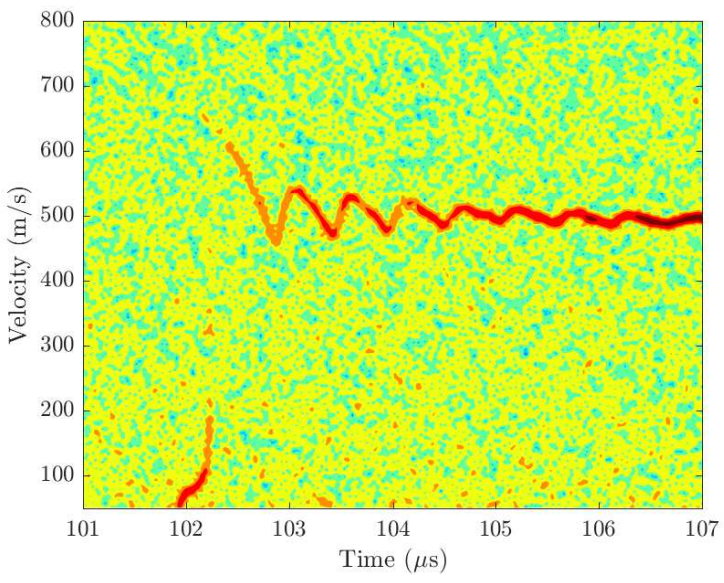

Figure B.3: An aluminum 6061-T6 target impacted with an aluminum 6061-T6 flyer plate at a velocity of $667 \mathrm{~m} \cdot \mathrm{s}^{-1}$.

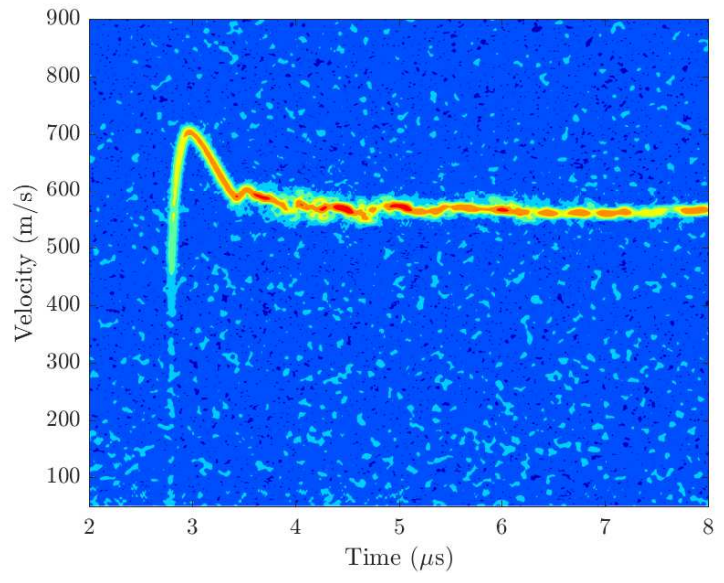

Figure B.5: A PMMA target impacted with an aluminum 6061-T6 flyer plate at a velocity of $502 \mathrm{~m} \cdot \mathrm{s}^{-1}$.

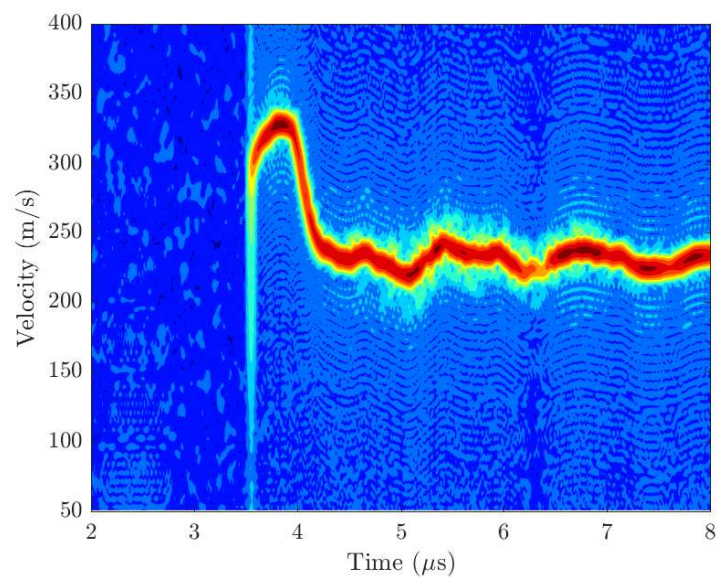

Figure B.4: A PMMA target impacted with an aluminum 6061-T6 flyer plate at a velocity of $208 \mathrm{~m} \cdot \mathrm{s}^{-1}$.

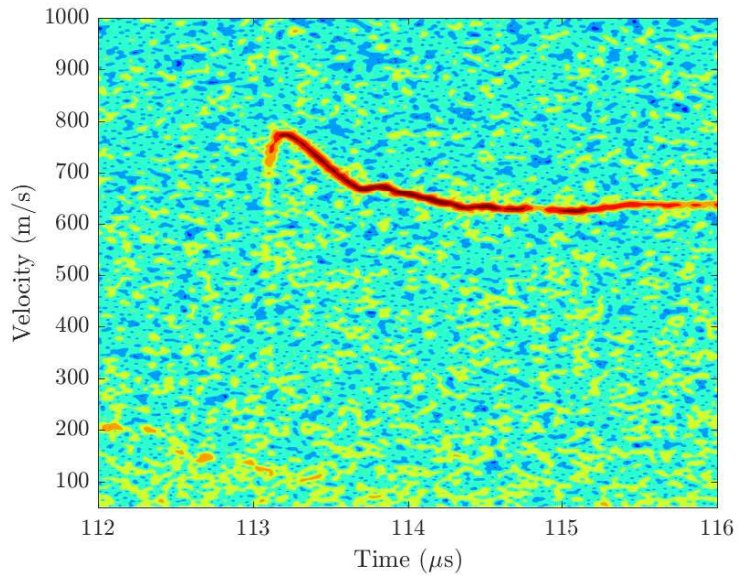

Figure B.6: A PMMA target impacted with an aluminum 6061-T6 flyer plate at a velocity of $606 \mathrm{~m} \cdot \mathrm{s}^{-1}$. 


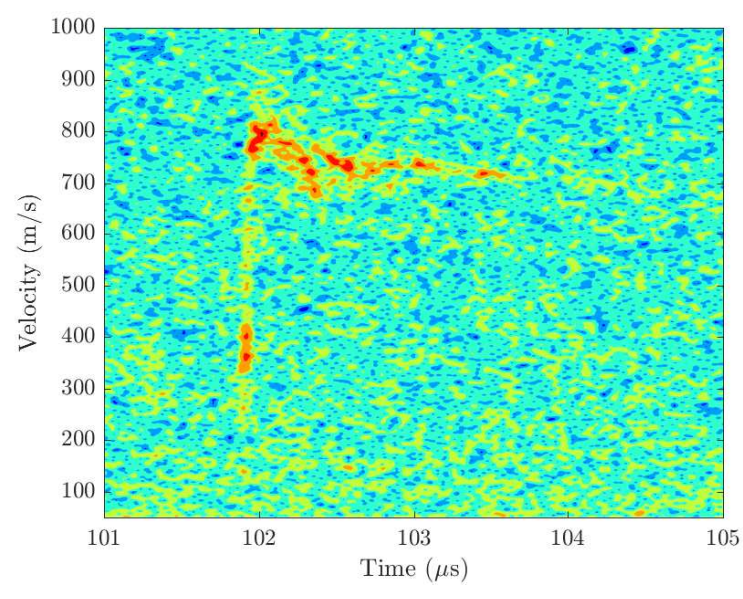

Figure B.7: A PMMA target impacted with an aluminum 6061-T6 flyer plate at a velocity of $656 \mathrm{~m} \cdot \mathrm{s}^{-1}$.

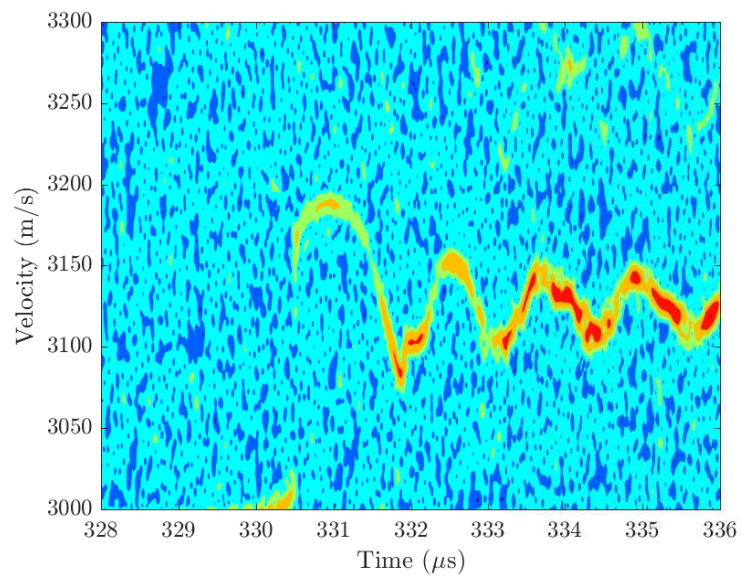

Figure B.9: A PMMA target impacted with a PMMA flyer plate at a velocity of $203 \mathrm{~m} \cdot \mathrm{s}^{-1}$. A baseline velocity offset of $2990 \mathrm{~m} \cdot \mathrm{s}^{-1}$ was applied.

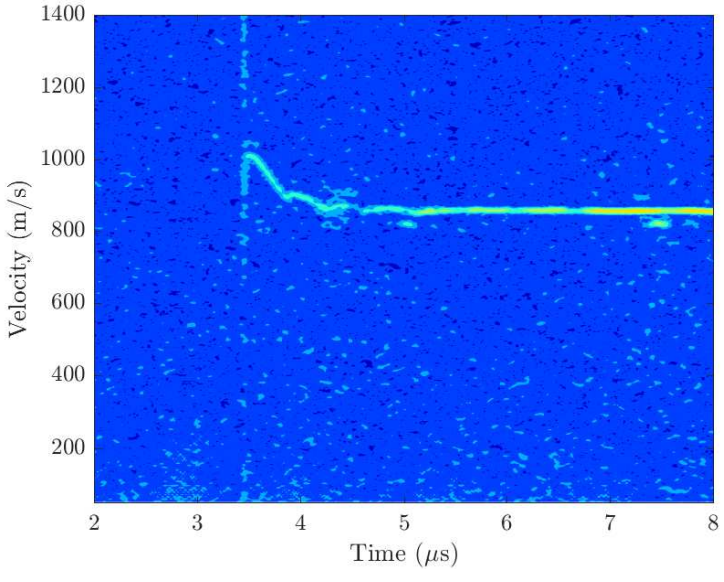

Figure B.8: A PMMA target impacted with an aluminum 6061-T6 flyer plate at a velocity of $722 \mathrm{~m} \cdot \mathrm{s}^{-1}$.

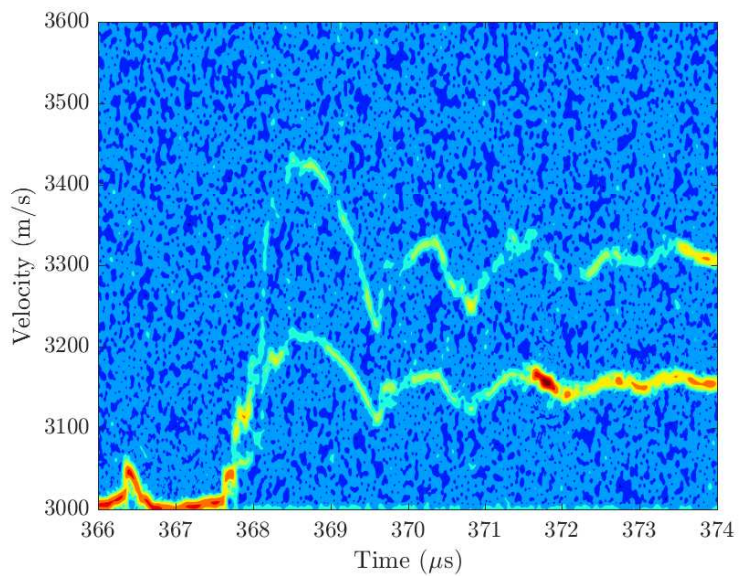

Figure B.10: A PMMA target impacted with a PMMA flyer plate at a velocity of 252 $\mathrm{m} \cdot \mathrm{s}^{-1}$. A baseline velocity offset of 3004 $\mathrm{m} \cdot \mathrm{s}^{-1}$ was applied. 


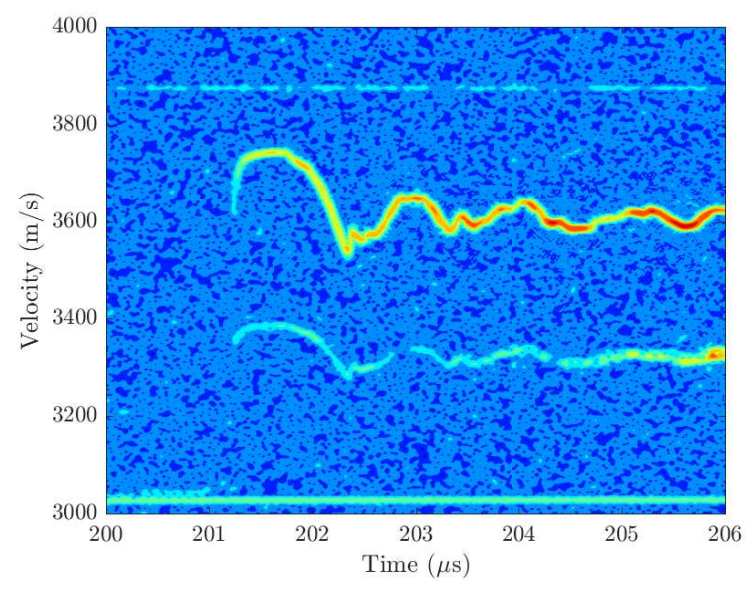

Figure B.11: A PMMA target impacted with a PMMA flyer plate at a velocity of 357 $\mathrm{m} \cdot \mathrm{s}^{-1}$. A baseline velocity offset of 3027 $\mathrm{m} \cdot \mathrm{s}^{-1}$ was applied.

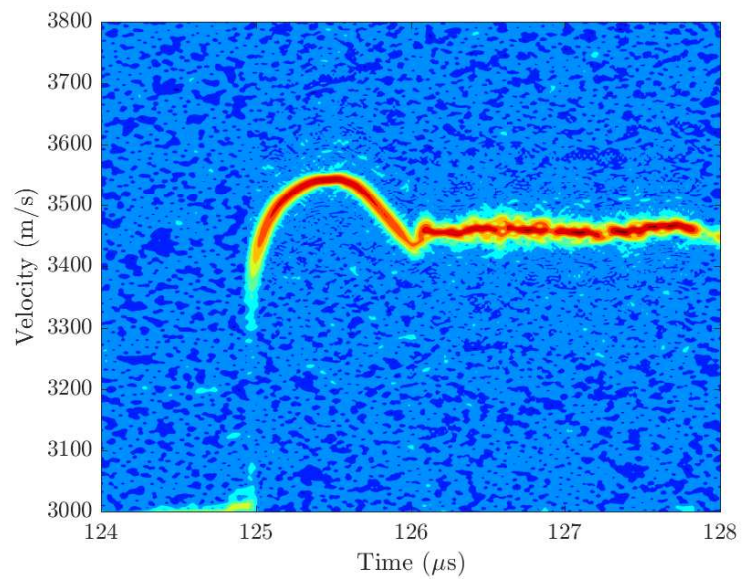

Figure B.13: A PMMA target impacted with a PMMA flyer plate at a velocity of 563 $\mathrm{m} \cdot \mathrm{s}^{-1}$.

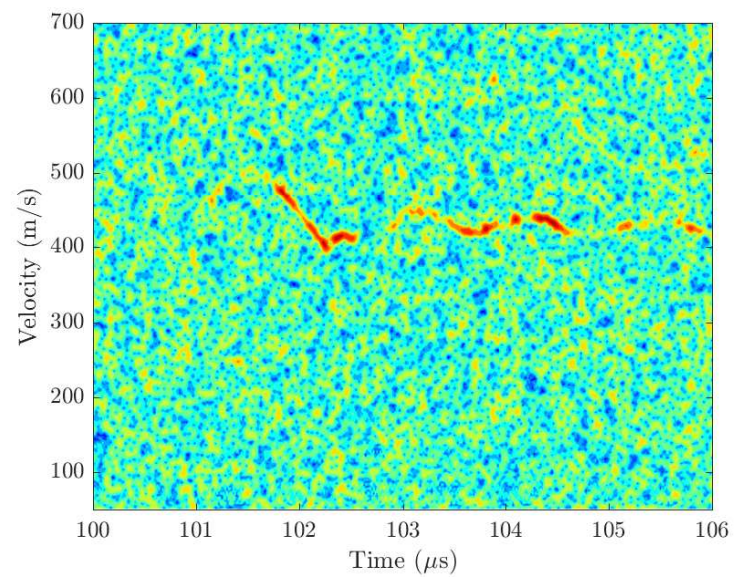

Figure B.12: A PMMA target impacted with a PMMA flyer plate at a velocity of 498 $\mathrm{m} \cdot \mathrm{s}^{-1}$.

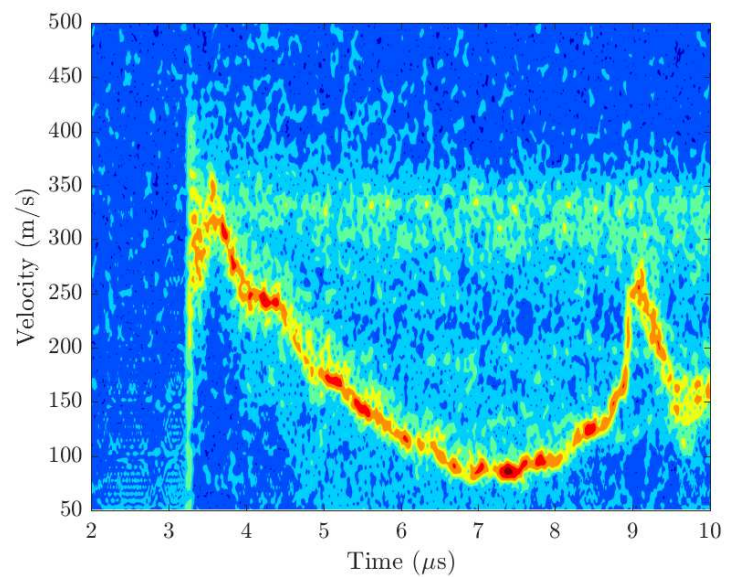

Figure B.14: An EPON 828 / EPIKURE 3223 epoxy target impacted with an aluminum 6061-T6 flyer plate at a velocity of $219 \mathrm{~m} \cdot \mathrm{s}^{-1}$. 


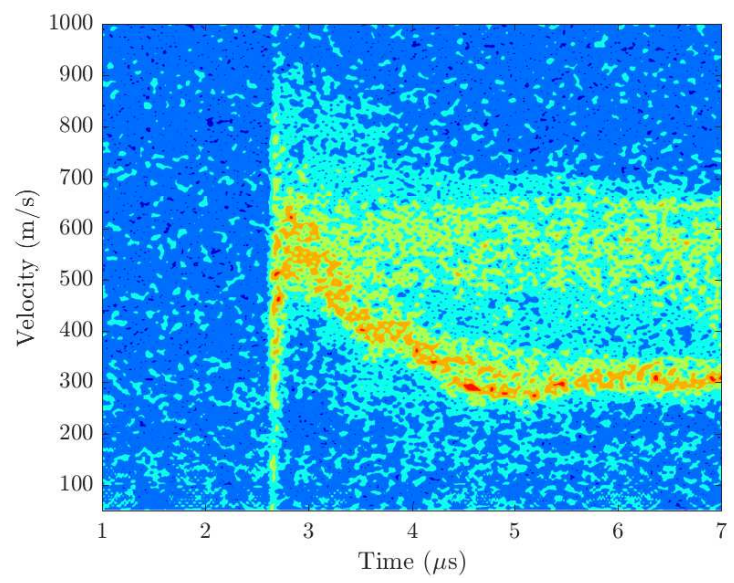

Figure B.15: An EPON 828 / EPIKURE 3223 epoxy target impacted with an aluminum 6061-T6 flyer plate at a velocity of $363 \mathrm{~m} \cdot \mathrm{s}^{-1}$.

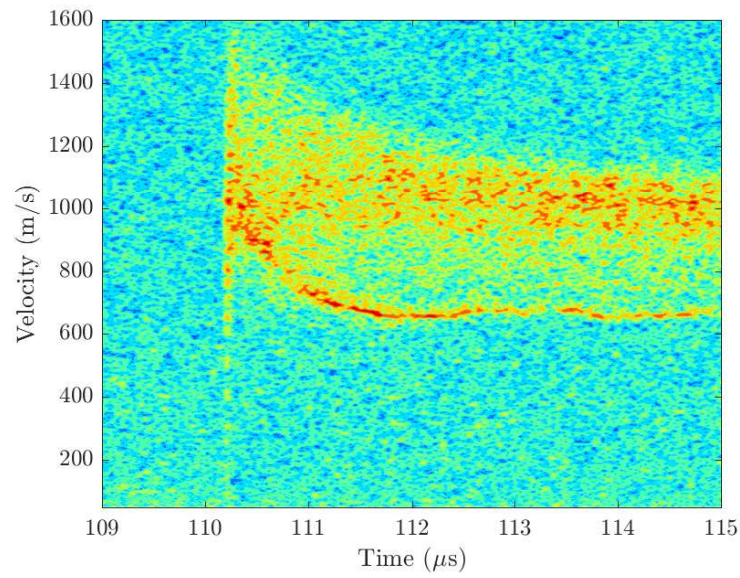

Figure B.17: An EPON 828 / EPIKURE 3223 epoxy target impacted with an aluminum 6061-T6 flyer plate at a velocity of $671 \mathrm{~m} \cdot \mathrm{s}^{-1}$.

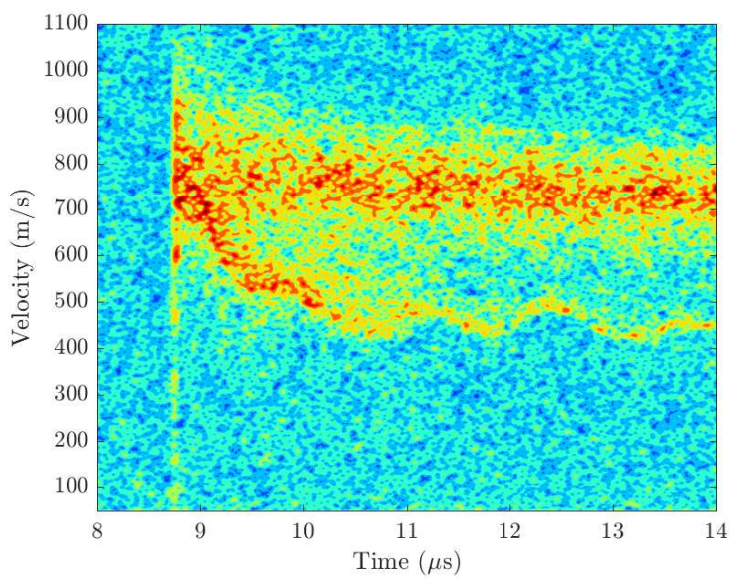

Figure B.16: An EPON 828 / EPIKURE 3223 epoxy target impacted with an aluminum 6061-T6 flyer plate at a velocity of $503 \mathrm{~m} \cdot \mathrm{s}^{-1}$.

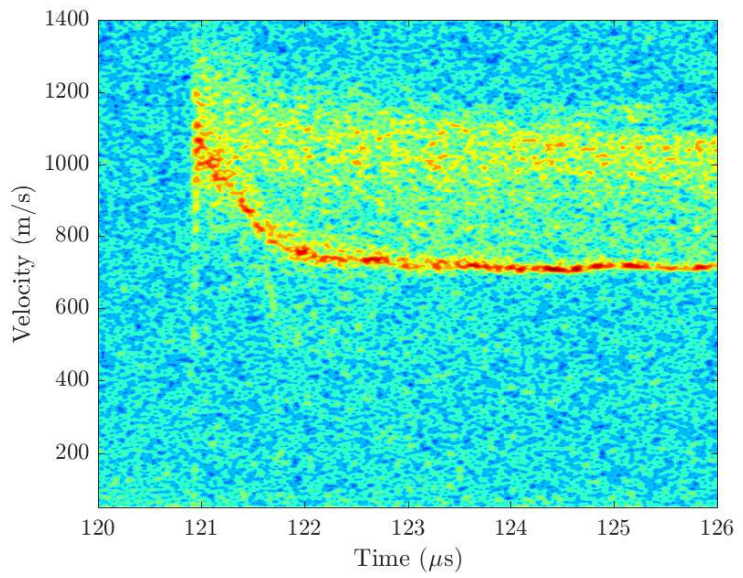

Figure B.18: An EPON 828 / EPIKURE 3223 epoxy target impacted with an aluminum 6061-T6 flyer plate at a velocity of $715 \mathrm{~m} \cdot \mathrm{s}^{-1}$. 


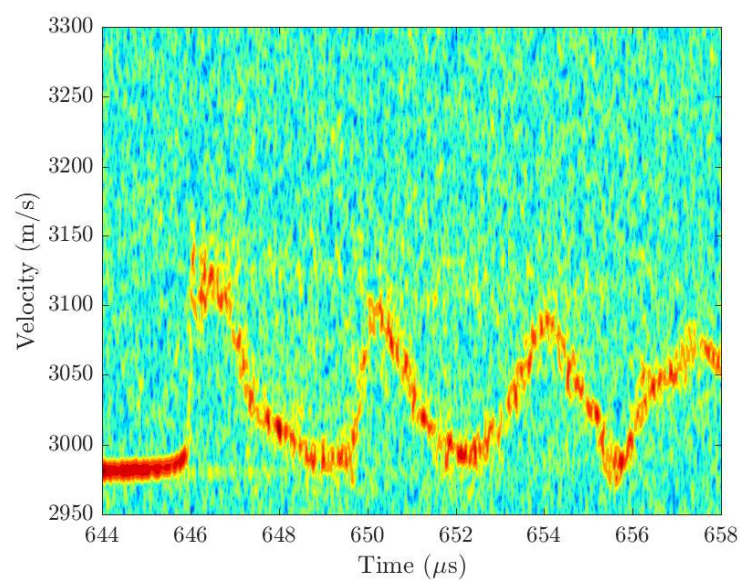

Figure B.19: An EPON 828 / EPIKURE 3223 epoxy target impacted with a PMMA flyer plate at a velocity of $136 \mathrm{~m} \cdot \mathrm{s}^{-1}$. A baseline velocity offset of $2984 \mathrm{~m} \cdot \mathrm{s}^{-1}$ was applied.

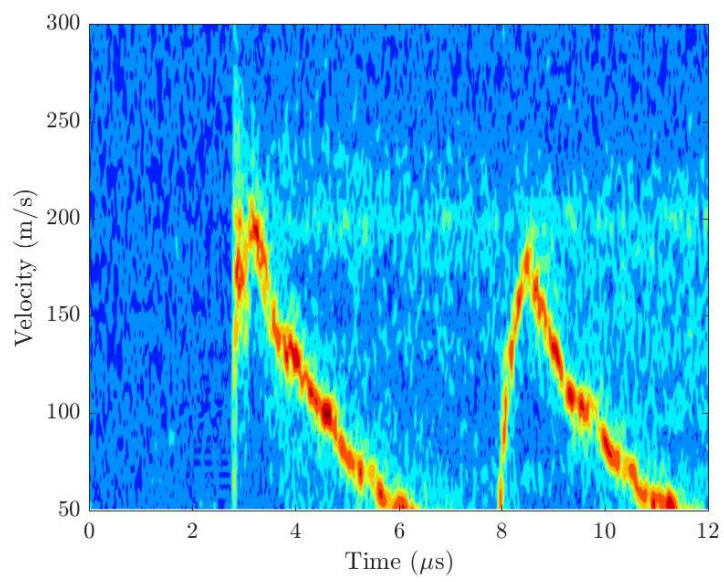

Figure B.21: An EPON 828 / EPIKURE 3233 epoxy target impacted with an aluminum 6061-T6 flyer plate at a velocity of $118 \mathrm{~m} \cdot \mathrm{s}^{-1}$.

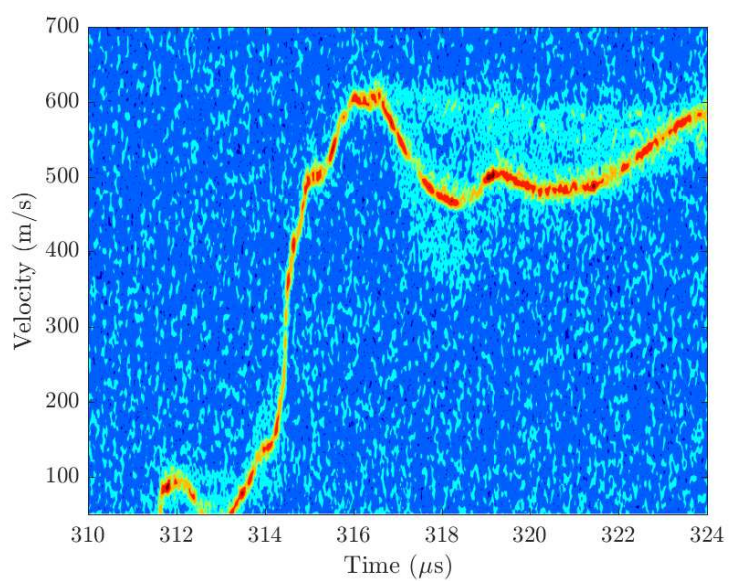

Figure B.20: An EPON 828 / EPIKURE 3223 epoxy target impacted with a PMMA flyer plate at a velocity of $585 \mathrm{~m} \cdot \mathrm{s}^{-1}$.

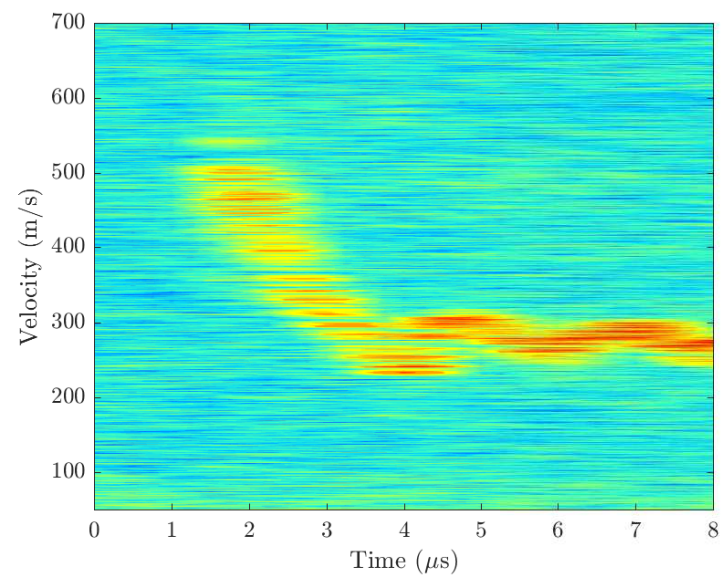

Figure B.22: An EPON 828 / EPIKURE 3233 epoxy target impacted with an aluminum 6061-T6 flyer plate at a velocity of $318 \mathrm{~m} \cdot \mathrm{s}^{-1}$. 


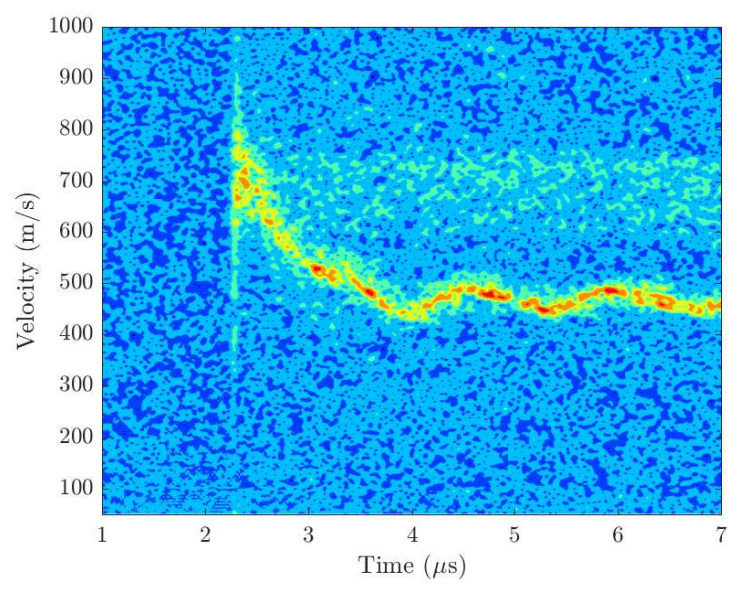

Figure B.23: An EPON 828 / EPIKURE 3233 epoxy target impacted with an aluminum 6061-T6 flyer plate at a velocity of $471 \mathrm{~m} \cdot \mathrm{s}^{-1}$.

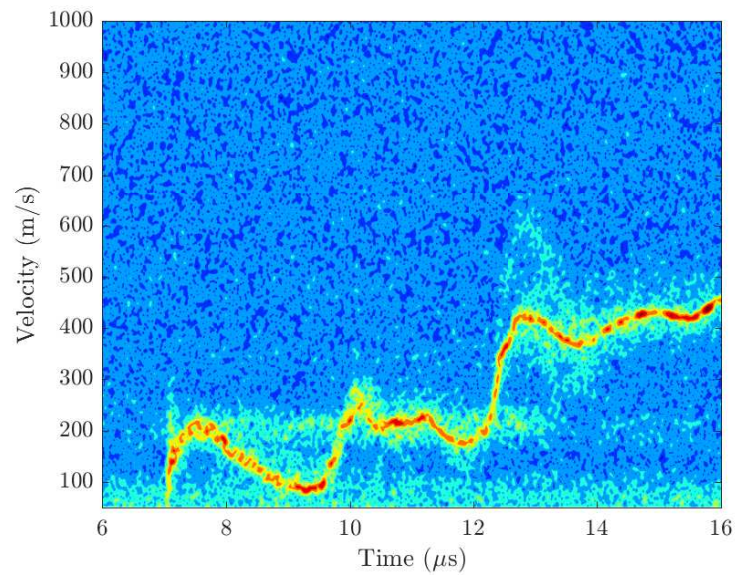

Figure B.25: An EPON 828 / EPIKURE 3233 epoxy target impacted with a PMMA flyer plate at a velocity of $484 \mathrm{~m} \cdot \mathrm{s}^{-1}$.

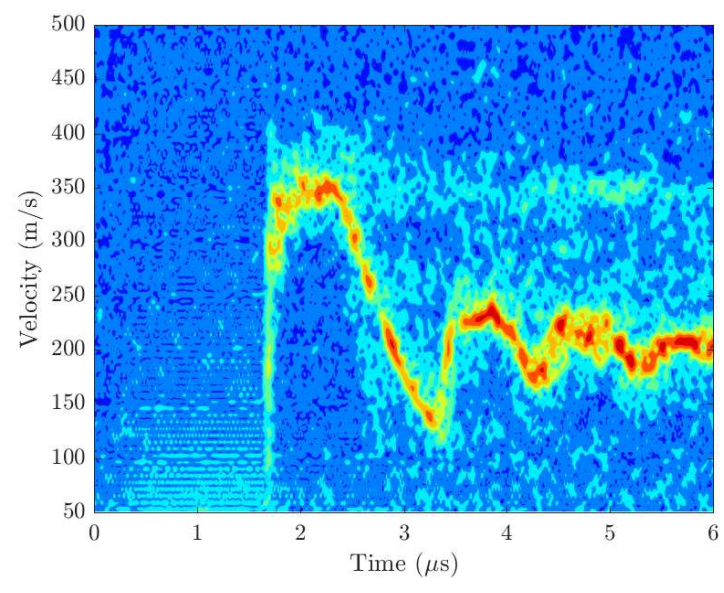

Figure B.24: An EPON 828 / EPIKURE 3233 epoxy target impacted with a PMMA flyer plate at a velocity of $344 \mathrm{~m} \cdot \mathrm{s}^{-1}$.

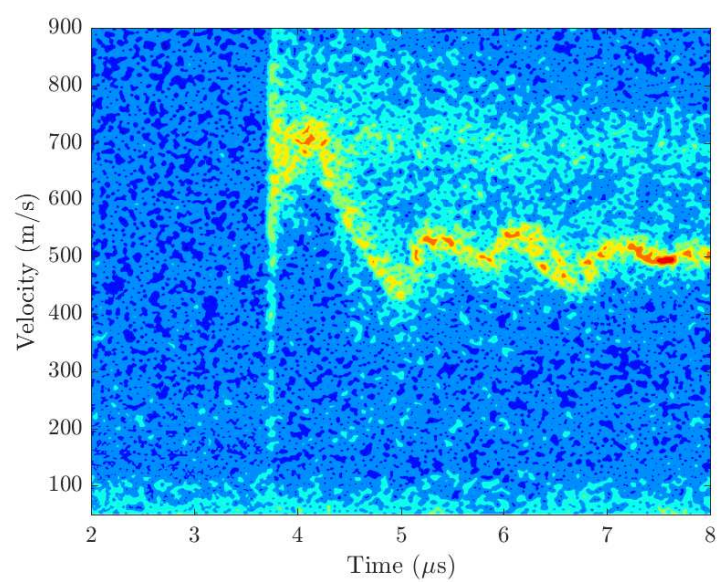

Figure B.26: An EPON 828 / EPIKURE 3233 epoxy target impacted with a PMMA flyer plate at a velocity of $638 \mathrm{~m} \cdot \mathrm{s}^{-1}$. 


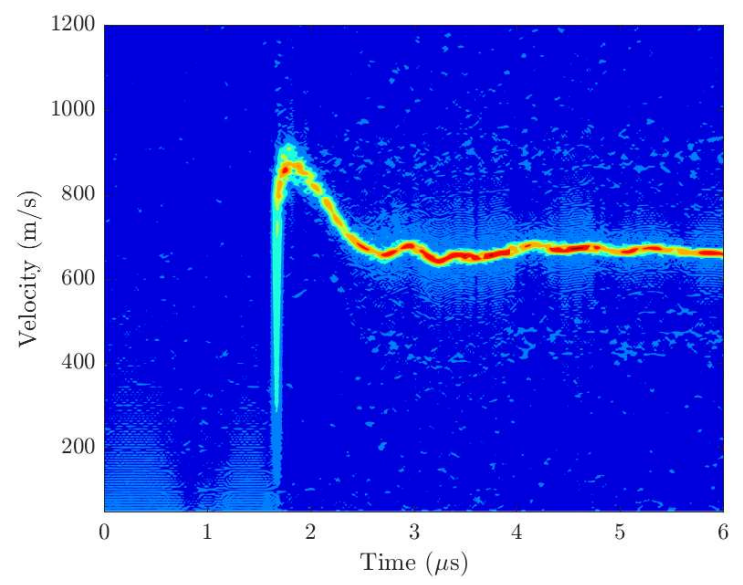

Figure B.27: An EPON 828 / EPIKURE 3223 epoxy +1 wt.\% P-CNT composite target impacted with an aluminum 6061-T6 flyer plate at a velocity of $617 \mathrm{~m} \cdot \mathrm{s}^{-1}$.

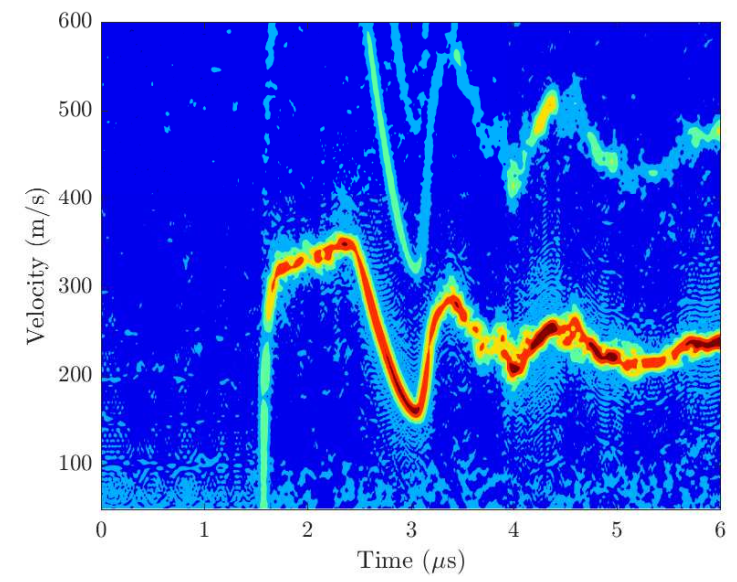

Figure B.29: An EPON 828 / EPIKURE 3223 epoxy +1 wt.\% P-CNT composite target impacted with a PMMA flyer plate at a velocity of $353 \mathrm{~m} \cdot \mathrm{s}^{-1}$.

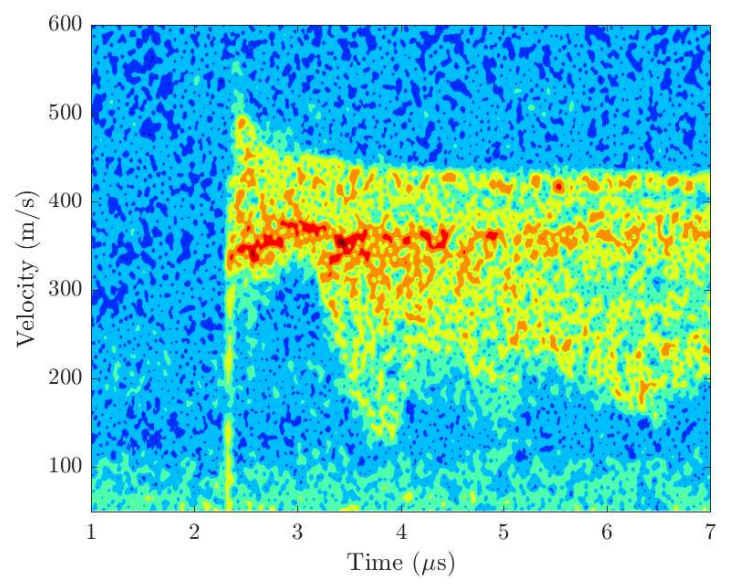

Figure B.28: An EPON 828 / EPIKURE 3223 epoxy +1 wt. $\%$ P-CNT composite target impacted with a PMMA flyer plate at a velocity of $346 \mathrm{~m} \cdot \mathrm{s}^{-1}$.

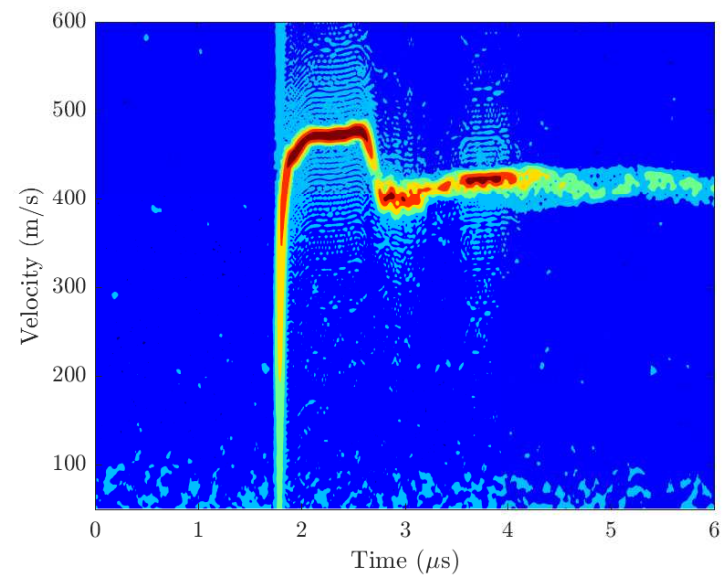

Figure B.30: An EPON 828 / EPIKURE 3223 epoxy +1 wt.\% P-CNT composite target impacted with a PMMA flyer plate at a velocity of $483 \mathrm{~m} \cdot \mathrm{s}^{-1}$. 


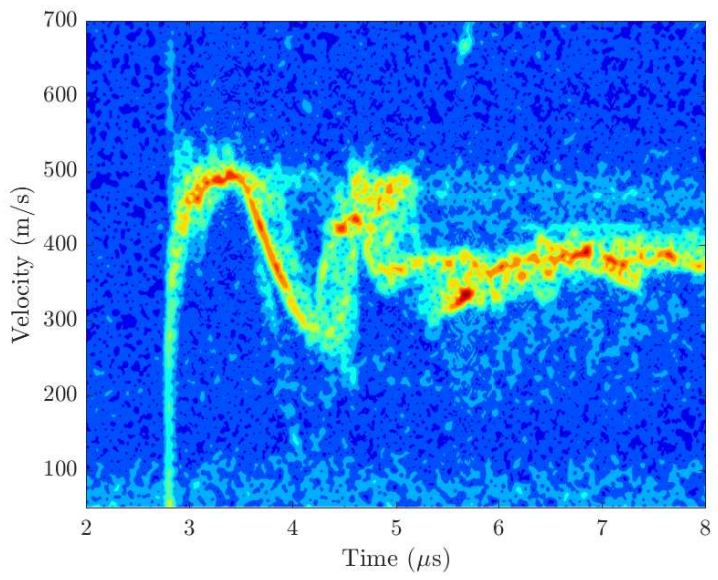

Figure B.31: An EPON 828 / EPIKURE 3223 epoxy + 1 wt.\% P-CNT composite target impacted with a PMMA flyer plate at a velocity of $499 \mathrm{~m} \cdot \mathrm{s}^{-1}$.

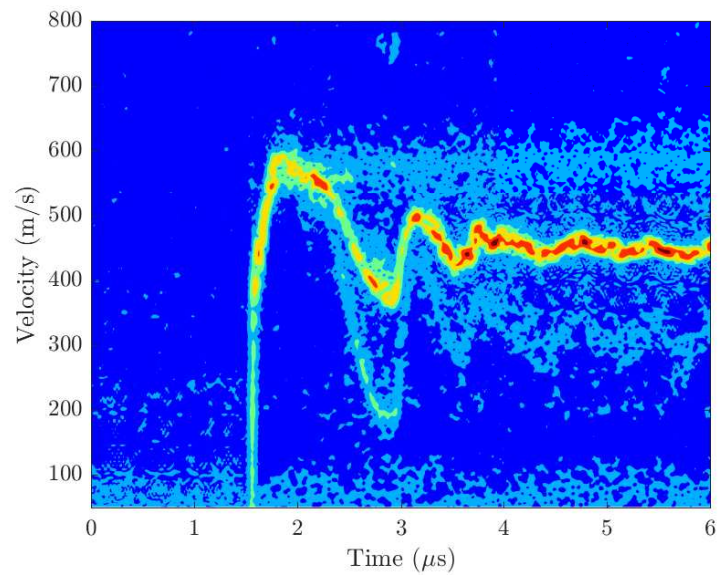

Figure B.32: An EPON 828 / EPIKURE 3223 epoxy +1 wt. $\%$ P-CNT composite target impacted with a PMMA flyer plate at a velocity of $625 \mathrm{~m} \cdot \mathrm{s}^{-1}$. 


\section{Appendix C}

\section{SEM Image Collection}

This appendix contains the complete collection of scanning electron microscope (SEM) images taken of fragments recovered from the plate impact experiments conducted with epoxy/carbon nanotube composite targets. A linear scale is provided in each image. These images were taken at the laboratory facilities of the National Research Council of Canada (NRC).

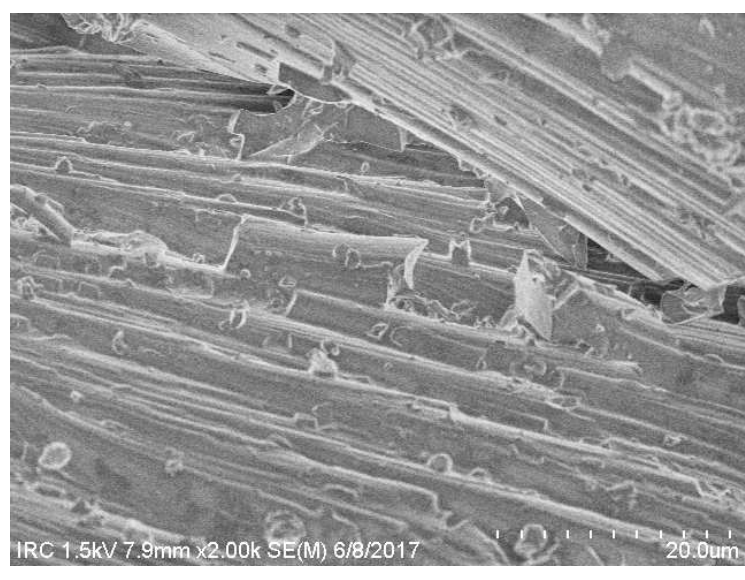

Figure C.1: SEM of a recovered EPON 828 / EPIKURE $3223+1 \mathrm{wt} . \%$ P-CNT target.

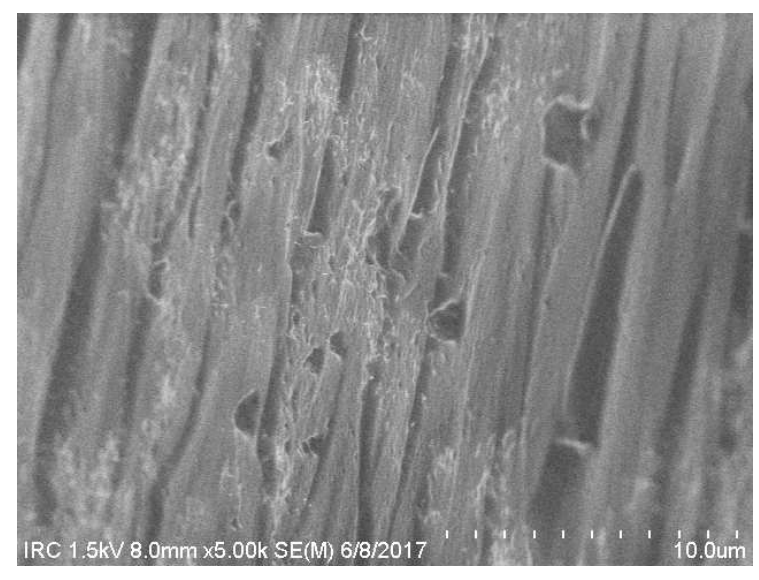

Figure C.2: SEM of a recovered EPON 828/ EPIKURE $3223+1 \mathrm{wt} . \%$ P-CNT target. 


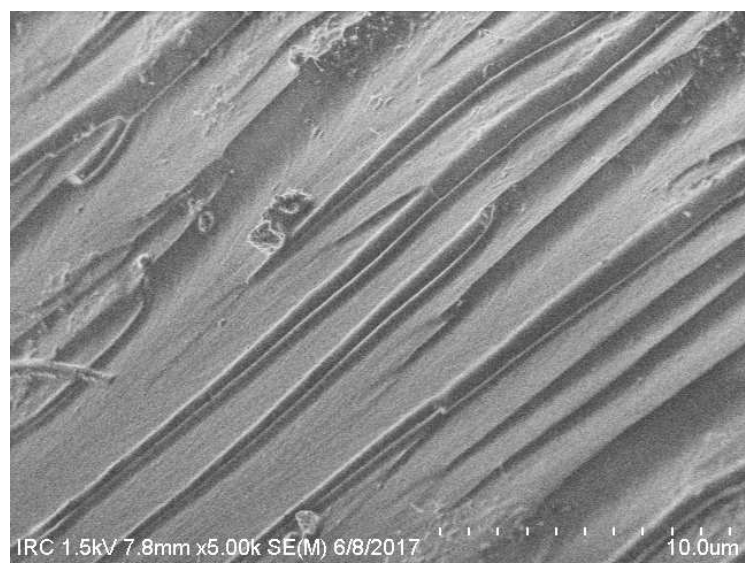

Figure C.3: SEM of a recovered EPON 828 / EPIKURE $3223+1 \mathrm{wt} . \%$ P-CNT target.

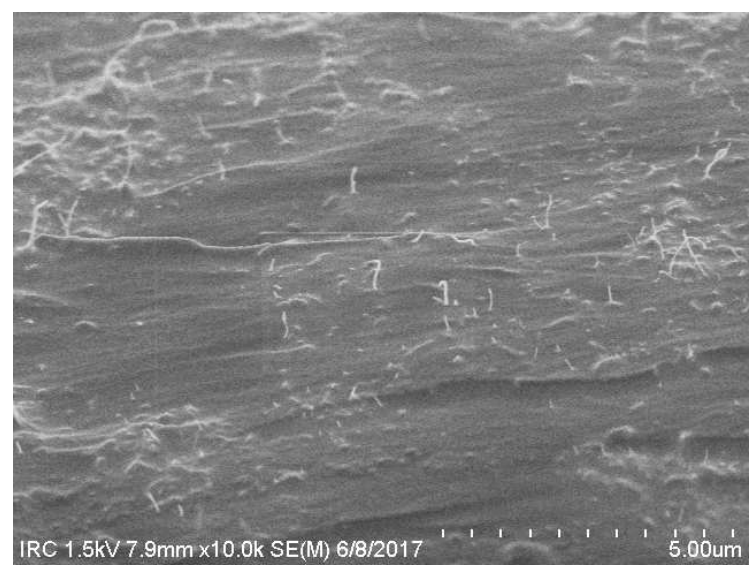

Figure C.5: SEM of a recovered EPON 828 / EPIKURE $3223+1$ wt. \% P-CNT target.

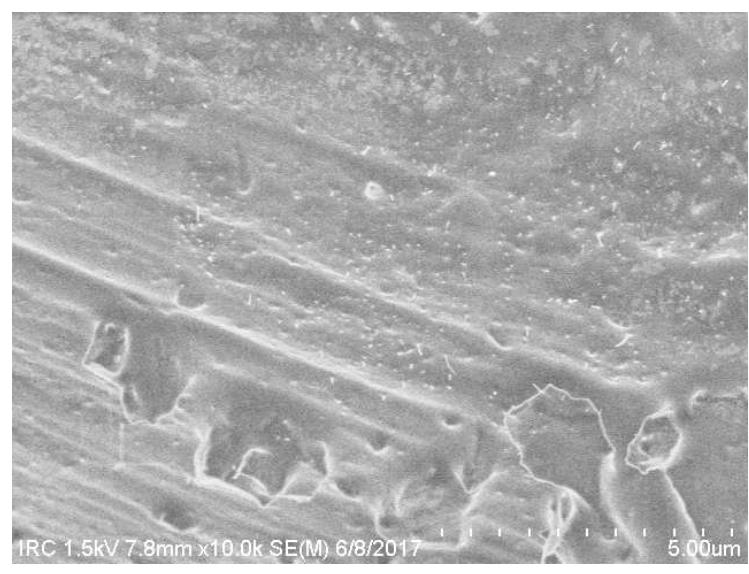

Figure C.7: SEM of a recovered EPON 828 / EPIKURE $3223+1 \mathrm{wt} . \%$ P-CNT target.

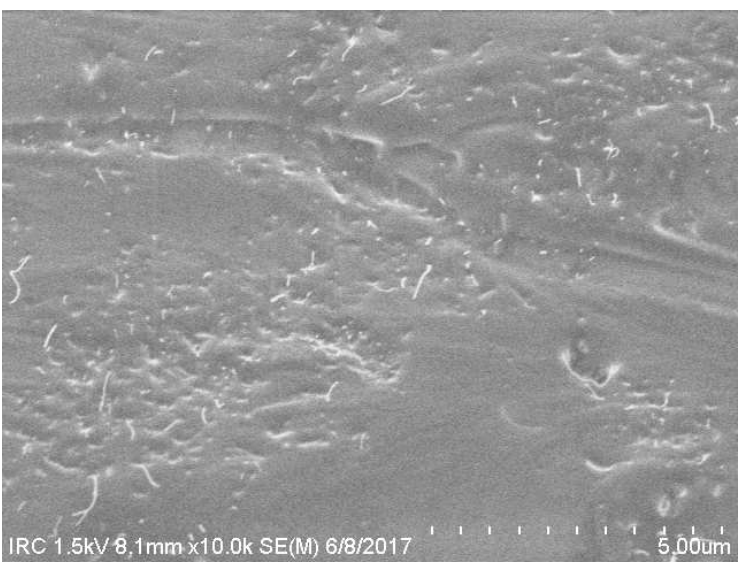

Figure C.4: SEM of a recovered EPON 828 / EPIKURE $3223+1 \mathrm{wt} . \%$ P-CNT target.

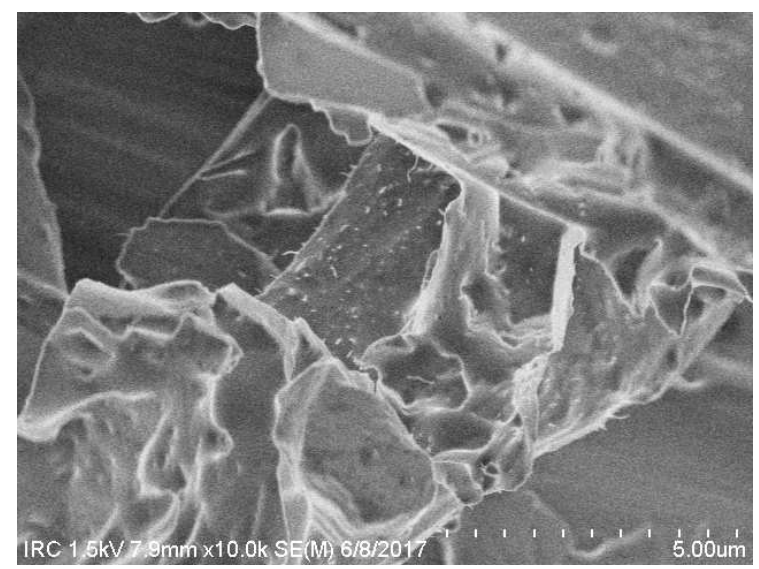

Figure C.6: SEM of a recovered EPON 828 / EPIKURE $3223+1 \mathrm{wt} . \%$ P-CNT target.

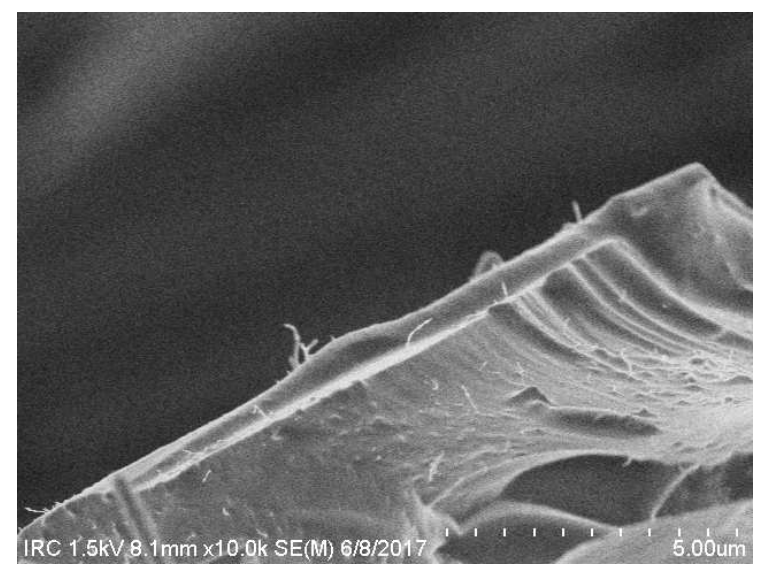

Figure C.8: SEM of a recovered EPON 828 / EPIKURE $3223+1 \mathrm{wt} . \%$ P-CNT target. 


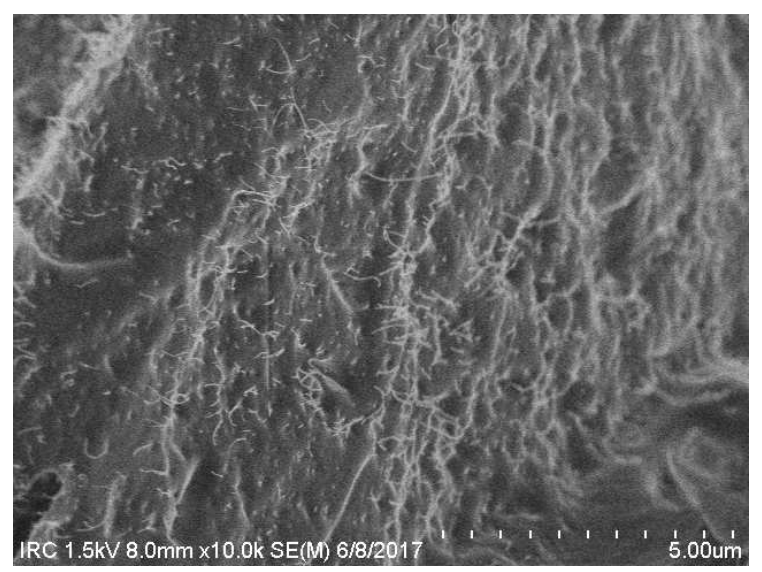

Figure C.9: SEM of a recovered EPON 828 / EPIKURE $3223+1 \mathrm{wt} . \%$ P-CNT target.

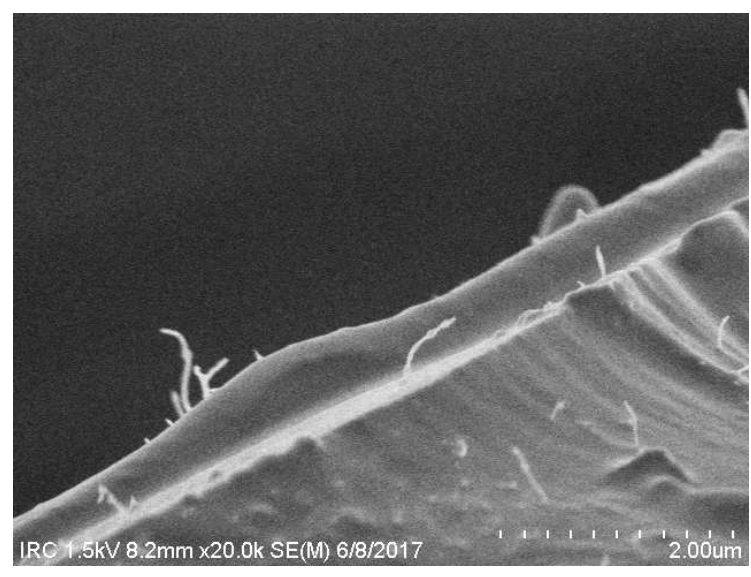

Figure C.11: SEM of a recovered EPON 828 / EPIKURE $3223+1$ wt. \% P-CNT target.

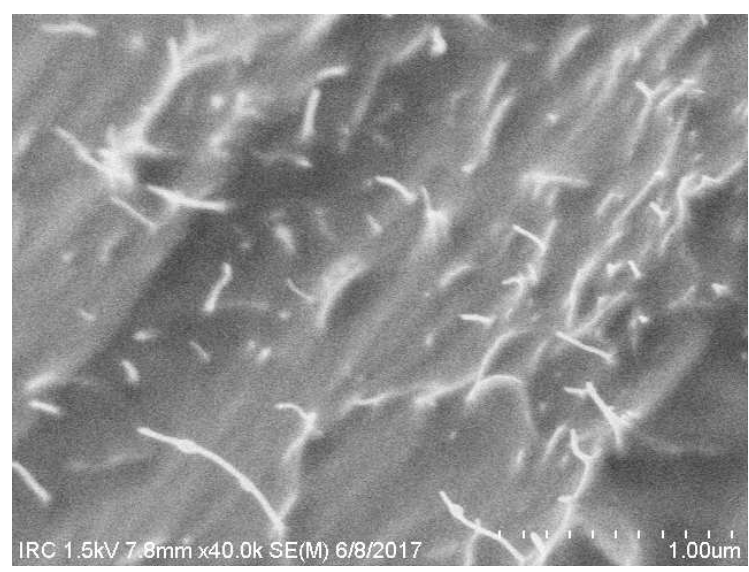

Figure C.13: SEM of a recovered EPON 828 / EPIKURE $3223+1$ wt. \% P-CNT target.

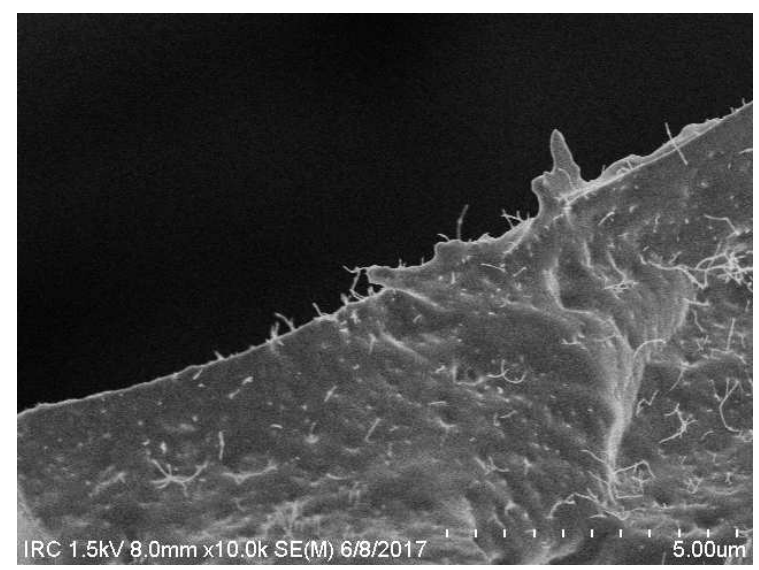

Figure C.10: SEM of a recovered EPON 828 / EPIKURE $3223+1$ wt.\% P-CNT target.

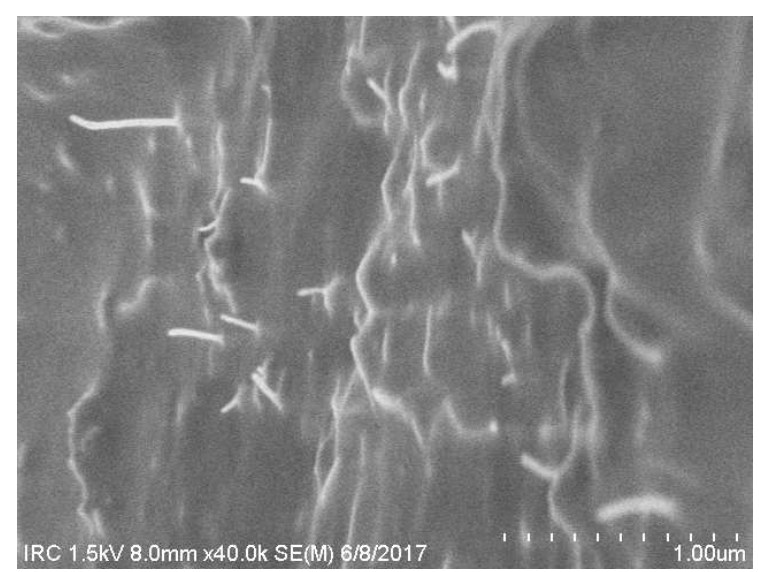

Figure C.12: SEM of a recovered EPON 828 / EPIKURE $3223+1$ wt.\% P-CNT target.

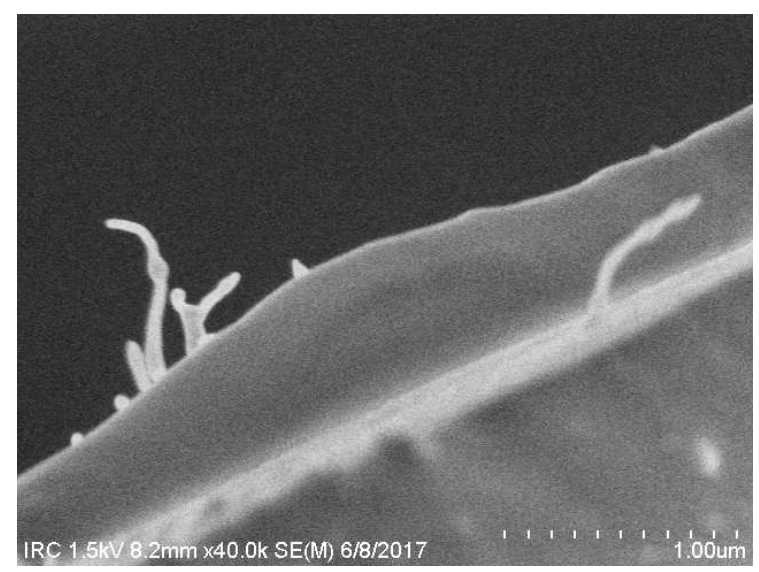

Figure C.14: SEM of a recovered EPON 828 / EPIKURE $3223+1$ wt.\% P-CNT target. 


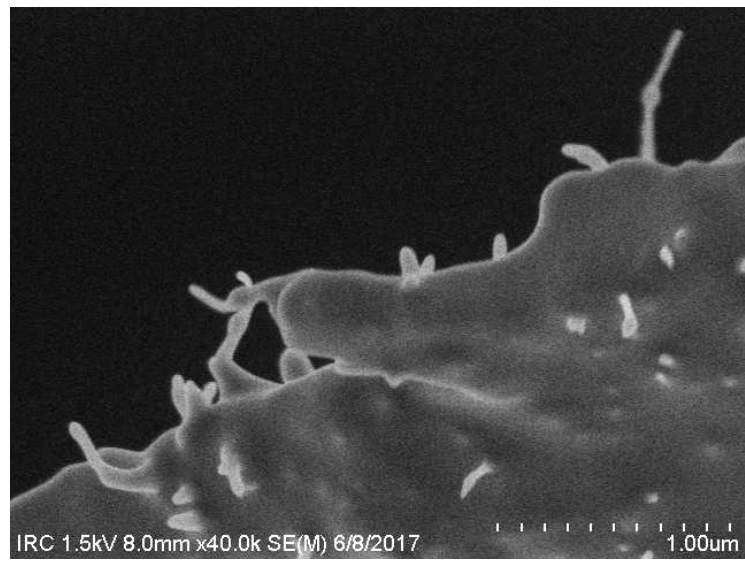

Figure C.15: SEM of a recovered EPON 828 / EPIKURE $3223+1$ wt. \% P-CNT target.

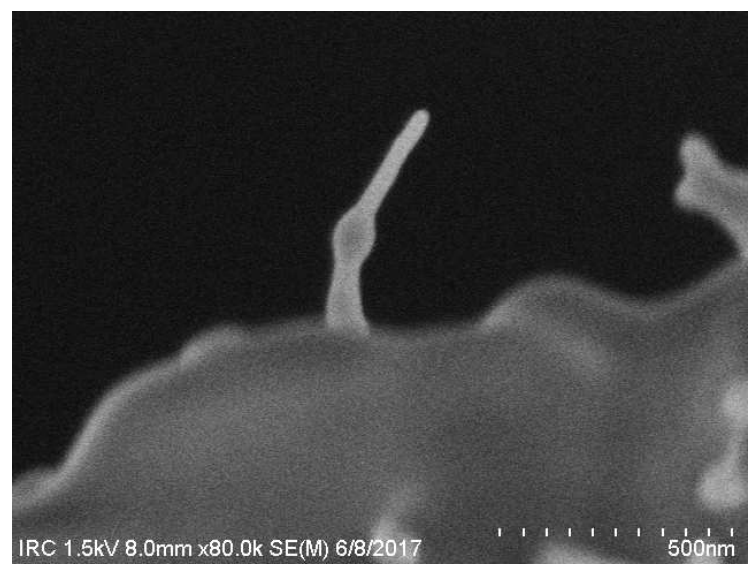

Figure C.17: SEM of a recovered EPON 828 / EPIKURE $3223+1$ wt.\% P-CNT target.

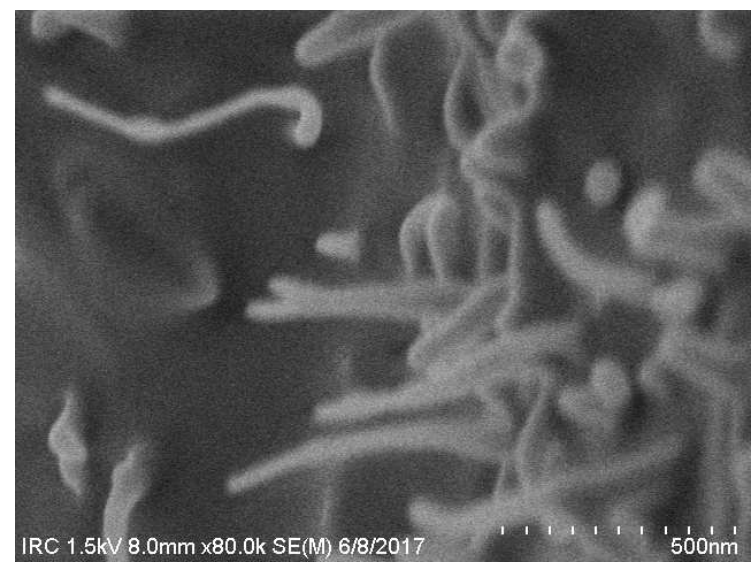

Figure C.16: SEM of a recovered EPON 828 / EPIKURE $3223+1$ wt.\% P-CNT target.

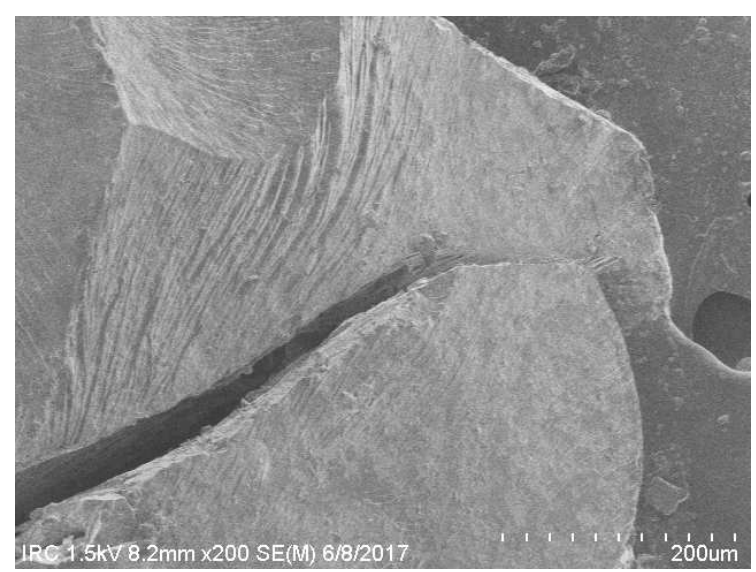

Figure C.18: SEM of a recovered EPON 828 / EPIKURE $3223+1$ wt.\% P-CNT target.

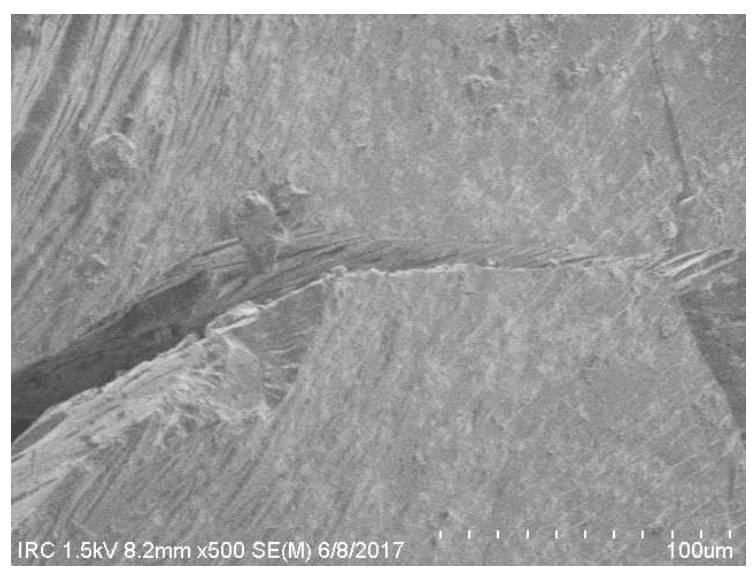

Figure C.19: SEM of a recovered EPON 828 / EPIKURE $3223+1$ wt.\% P-CNT target. 